\author{
Aus dem Fachbereich Medizin \\ der Johann Wolfgang Goethe-Universität \\ Frankfurt am Main
}

betreut an der

Klinik für Augenheilkunde

Direktor: Univ.-Prof. Dr. med. Thomas Kohnen

\title{
Vergleich von neun modernen Formeln zur Berechnung der Linsenstärke einer quadrifokalen Intraokularlinse
}

\author{
Dissertation \\ zur Erlangung des Doktorgrades der Medizin \\ des Fachbereichs Medizin \\ der Johann Wolfgang Goethe-Universität \\ Frankfurt am Main
}

vorgelegt von

Carolin Marion Kolb

aus Aschaffenburg

Frankfurt am Main, 2020 
Dekan:

Referent:

Korreferent:

Tag der mündlichen Prüfung:
Prof. Dr. Stefan Zeuzem

Prof. Dr. Thomas Kohnen

Prof. Dr. Oliver Schwenn

23. 08. 2021 
Für meine Eltern 


\section{Inhaltsverzeichnis}

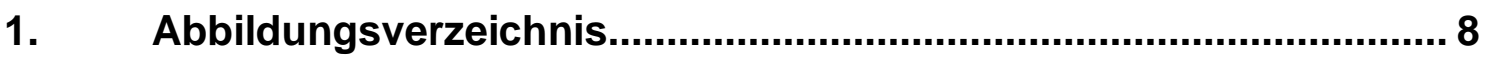

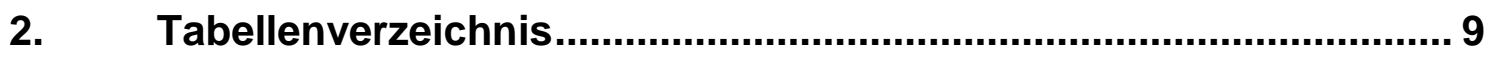

3. Abkürzungsverzeichnis............................................................... 10

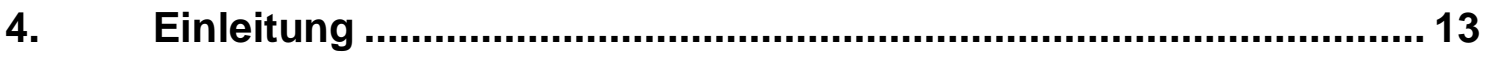

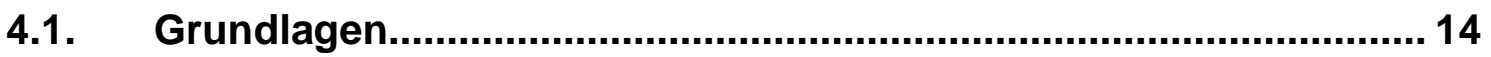

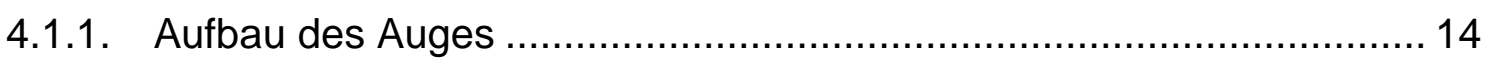

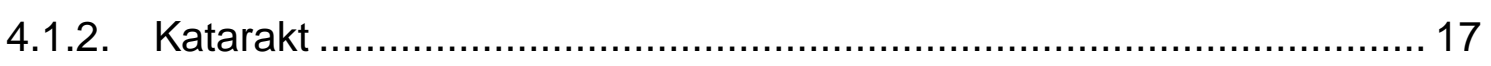

4.2. Durchführung der Kataraktoperation.............................................. 22

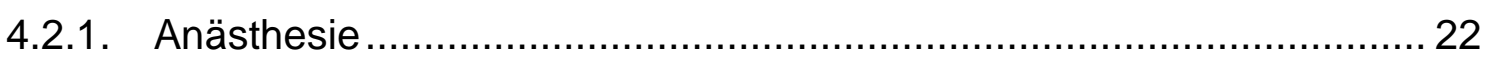

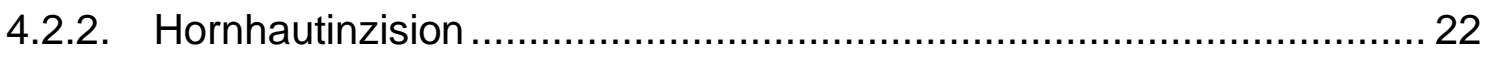

4.2.3. Entfernung der getrübten Linse ................................................... 23

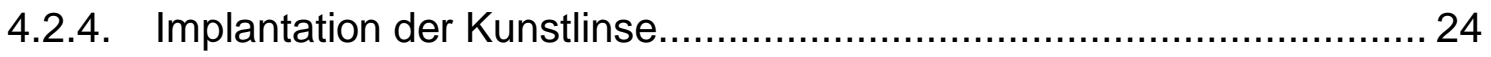

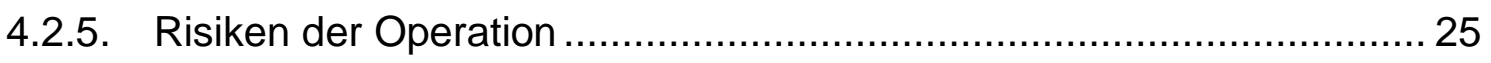

4.3. Einflüsse auf das Ergebnis der Kataraktchirurgie .......................... 27

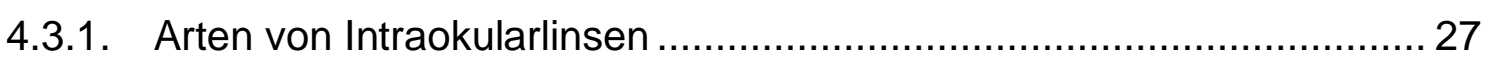

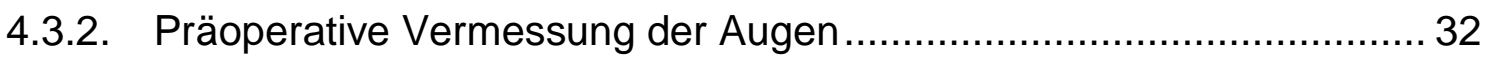

4.3.3. Formeln zur Kalkulation der Stärke der Intraokularlinse ...................... 35

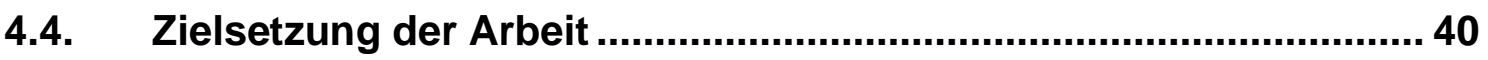

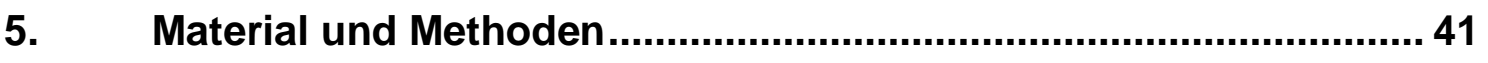

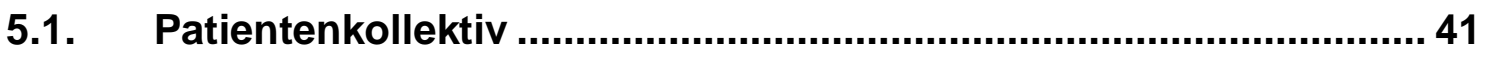

5.1.1. Studiendesign und Studienpopulation .......................................... 41

5.1.2. Einschluss- und Ausschlusskriterien..................................................... 41

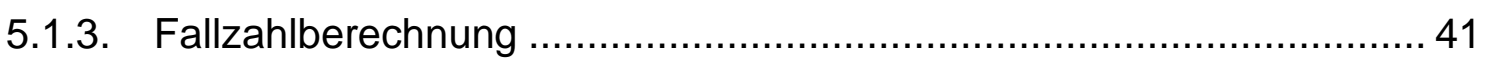

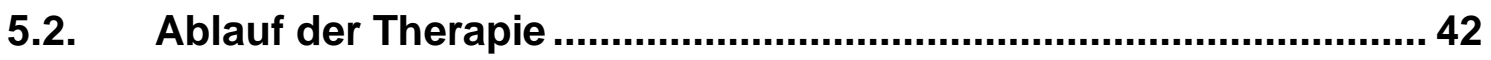

5.2.1. Präoperative Untersuchungen und Messungen ............................... 42

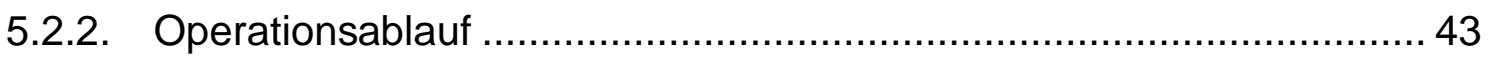

5.2.3. Postoperative Untersuchungen und Messungen ............................... 45 
5.3. Details der implantierten Intraokularlinse .................................... 45

5.4. Eigenschaften und Verfügbarkeit der Kalkulationsformeln ............ 48

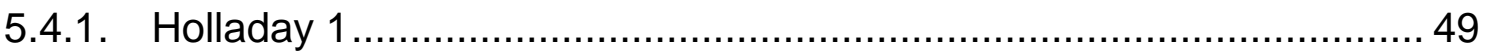

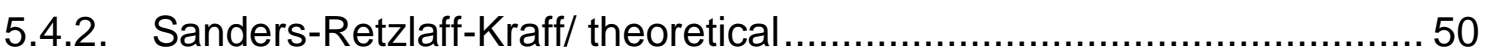

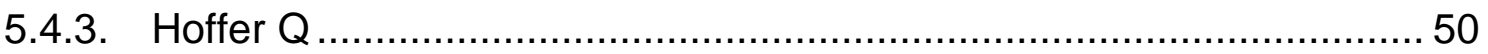

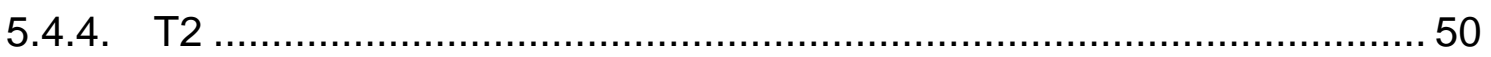

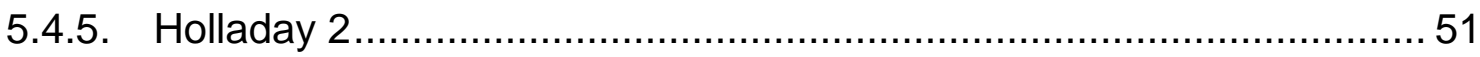

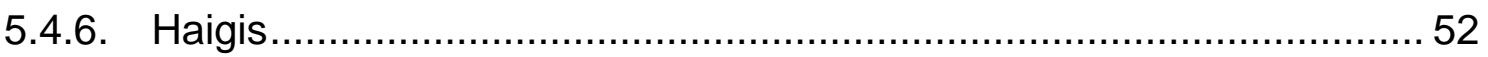

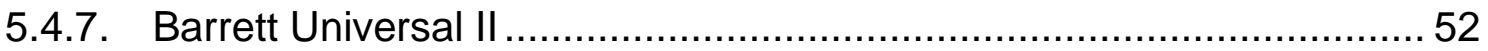

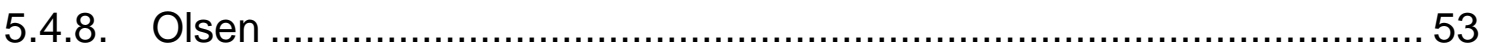

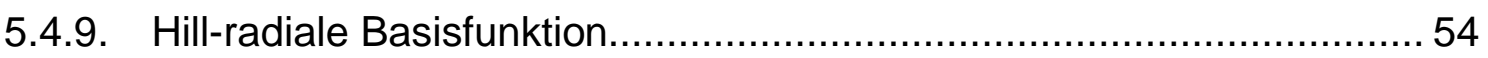

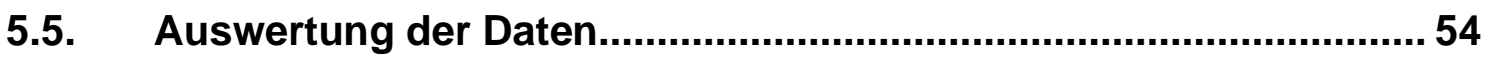

5.5.1. Einsetzen der Parameter in die Kalkulationsformeln ......................... 54

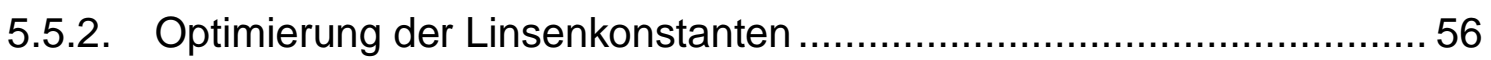

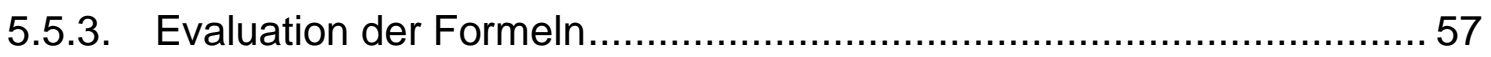

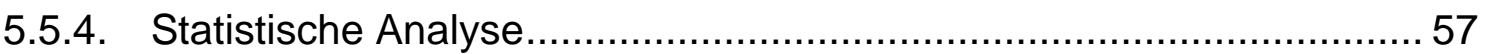

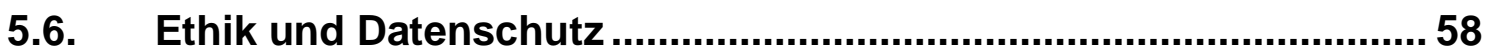

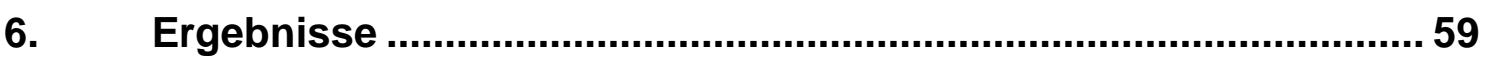

6.1. Charakteristik der Studienpopulation ............................................ 59

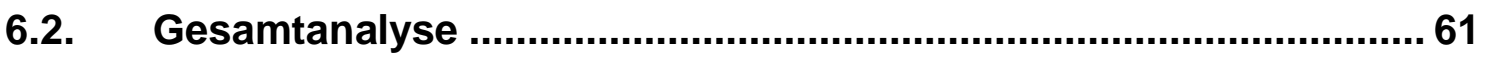

6.2.1. Mittlerer, medianer und maximaler absoluter Vorhersagefehler ..........61 61

6.2.2. Anteile der Augen innerhalb bestimmter Vorhersagefehler ................ 63

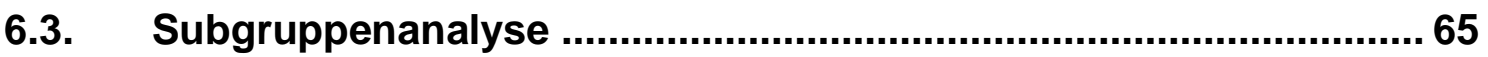

6.3.1. Mittlerer, medianer und maximaler absoluter Vorhersagefehler .......... 65

6.3.2. Anteile der Augen innerhalb bestimmter Vorhersagefehler ................. 68 


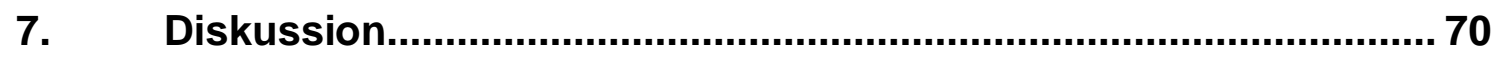

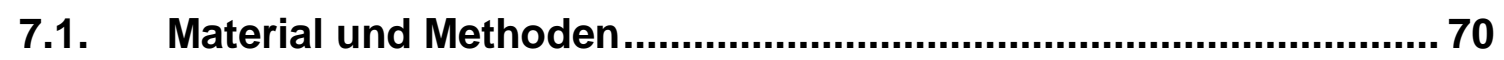

7.1.1. Vorgaben für Studien zum Vergleich von Kalkulationsformeln ............ 70

7.1.2. Messung der Hornhautbrechkraft .................................................. 72

7.1.3. Vorhersage der postoperativen Linsenposition .................................. 73

7.1.4. Umgang mit den optimierten Linsenkonstanten ................................ 76

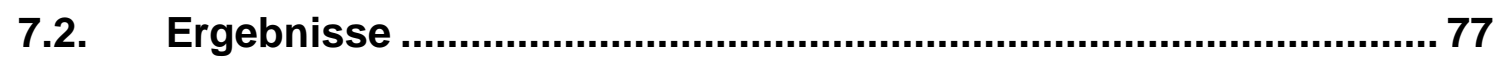

7.2.1. Aussagekraft der evaluierten Endpunkte ….................................... 77

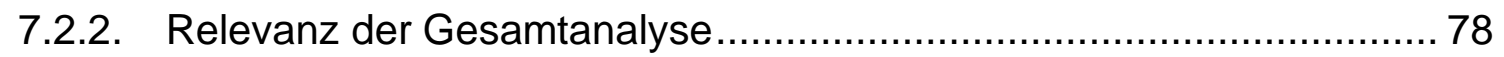

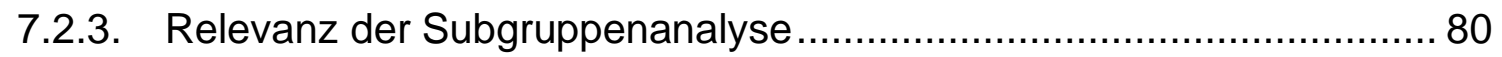

7.2.4. Kritische Betrachtung einzelner Patienten ....................................... 81

7.2.5. Vergleich der Sanders-Retzlaff-Kraff/ theoretical mit der T2 ............... 83

7.2.6. Besondere Aspekte der Hill-radiale Basisfunktion .............................. 85

7.3. Einordnung in den aktuellen Wissensstand..................................... 85

7.3.1. Linsenkalkulationsformeln für monofokale Linsen .............................. 86

7.3.2. Linsenkalkulationsformeln für multifokale Linsen ................................ 89

7.3.3. Problematik der Augen mit extremen Maßen..................................... 91

7.4. Verbesserung der visuellen Ergebnisse mit multifokalen Linsen durch die Verwendung geeigneterer Formeln.............................. 94

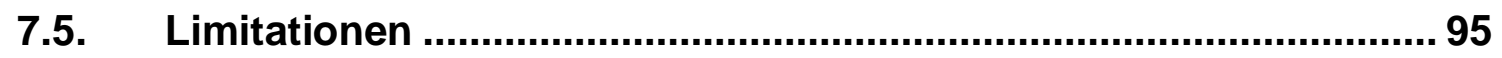

7.6. Schlussfolgerungen und Ausblick.................................................. 96

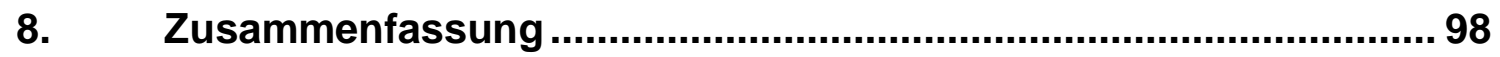

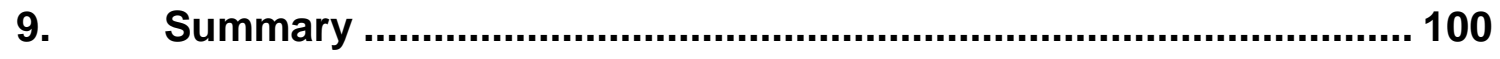

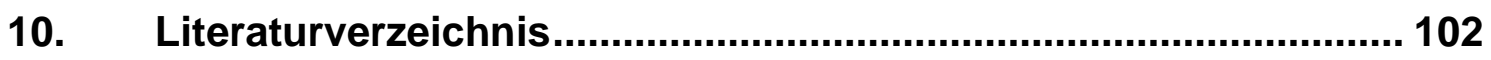




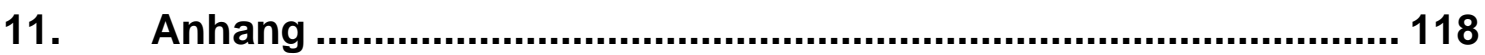

11.1. Details der publizierten Formeln ............................................... 118

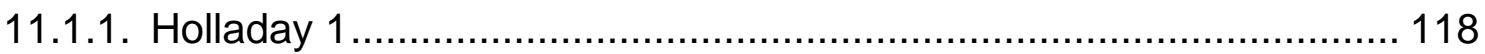

11.1.2. Sanders-Retzlaff-Kraff/ theoretical ............................................... 120

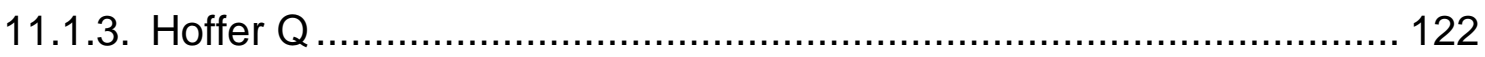

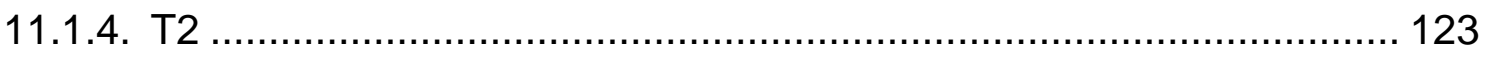

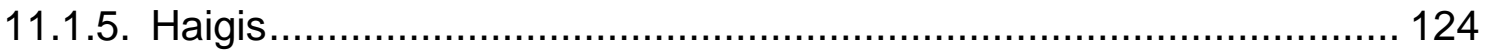

11.2. Ergebnisse der Cochran Q-Tests der Gesamtanalyse ................ 126

11.3. Vergleiche von jeweils zwei Formeln innerhalb der Subgruppen 127

12. Eigene Veröffentlichungen ...................................................... 128

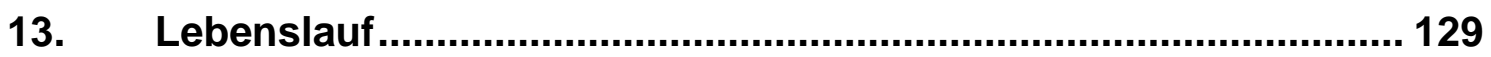

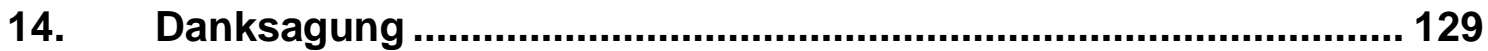

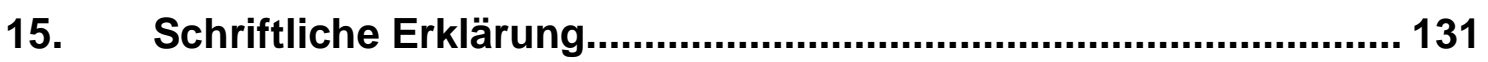




\section{Abbildungsverzeichnis}

Abbildung 1: Seitansicht des Auges ....................................................... 14

Abbildung 2: Histologischer Aufbau der Linse ........................................... 16

Abbildung 3: Darstellung der Messparameter .............................................. 33

Abbildung 4: Abmessungen des Vorderabschnitts im phaken und pseudophaken Auge ...................................................................... 36

Abbildung 5: Schnittführung der Linsenfragmentierung ………………....... 44

Abbildung 6: PanOptix-Intraokularlinse .................................................. 46

Abbildung 7: Vergleich der klassischen quadrifokalen Technologie mit der ENLIGHTEN-Technologie der PanOptix ................................. 47

Abbildung 8: Verteilung der Lichtenergie der PanOptix in Abhängigkeit von der Pupillenweite ................................................................ 47

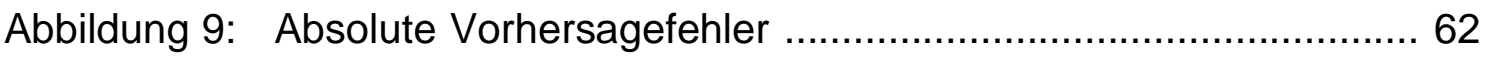

Abbildung 10: Anteile der Augen innerhalb bestimmter Vorhersagefehler ....... 64 Abbildung 11: Absolute Vorhersagefehler (Subgruppe mit Achsenlänge

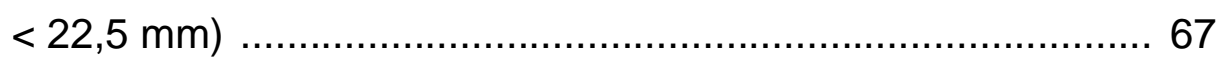

Abbildung 12: Absolute Vorhersagefehler (Subgruppe mit Achsenlänge $>25,5 \mathrm{~mm}$ )

Abbildung 13: Anteile der Augen innerhalb bestimmter Vorhersagefehler (Subgruppe mit Achsenlänge $<22,5 \mathrm{~mm}$ ) 68

Abbildung 14: Anteile der Augen innerhalb bestimmter Vorhersagefehler (Subgruppe mit Achsenlänge > 25,5 mm) 69

Abbildung 15: „Sanders-Retzlaff-Kraff/ theoretical cusp“ bei einer Achsenlänge von $23 \mathrm{~mm}$ 83

Abbildung 16: Bevorzugt verwendete Formeln laut Umfrage der European Society of Cataract and Refractive Surgery 94 


\section{Tabellenverzeichnis}

Tabelle 1: Einstellungen des Femtosekundenlasers .................................... 44

Tabelle 2: Einzusetzende Parameter für die Linsenkalkulationsformeln ........ 48

Tabelle 3: Empfohlene Linsenkonstanten für die PanOptix ............................. 55

Tabelle 4: Präoperative Werte der Studienpopulation ................................... 59

Tabelle 5: Postoperative Werte der Studienpopulation nach drei Monaten .... 60

Tabelle 6: Optimierte Linsenkonstanten für die PanOptix ............................... 60

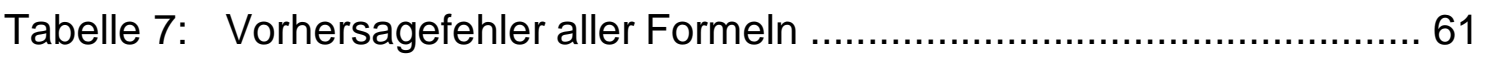

Tabelle 8: P-Werte der Vergleiche von jeweils zwei Formeln ......................... 63

Tabelle 9: Vorhersagefehler aller Formeln (Subgruppe mit Achsenlänge

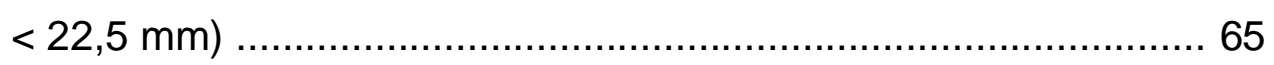

Tabelle 10: Vorhersagefehler aller Formeln (Subgruppe mit Achsenlänge

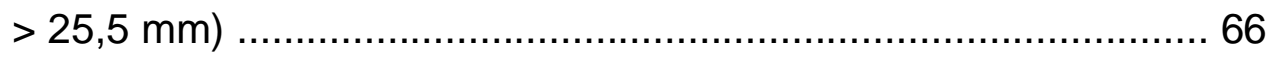

Tabelle 11: Gründe für refraktive Überraschungen bei der Linsenchirurgie ..... 82

Tabelle 12: Empfohlene Formeln bei unterschiedlichen Achsenlängen ........... 91

Tabelle 13: Vergleich der Anteile der Augen innerhalb eines Vorhersagefehlers

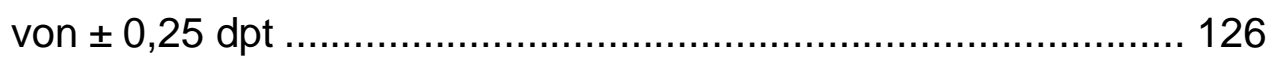

Tabelle 14: Vergleich der Anteile der Augen innerhalb eines Vorhersagefehlers von $\pm 0,5 \mathrm{dpt}$ 126

Tabelle 15: P-Werte der Vergleiche von jeweils zwei Formeln (Subgruppe mit Achsenlänge $<22,5 \mathrm{~mm}$ )

Tabelle 16: P-Werte der Vergleiche von jeweils zwei Formeln (Subgruppe mit Achsenlänge $>25,5 \mathrm{~mm}$ ) 127 


\section{Abkürzungsverzeichnis}

Aus Gründen der Verständlichkeit wurden in einigen Fällen die geläufigeren englischen Abkürzungen verwendet.
A
A-Konstante
$A C D$
Vorderkammertiefe (anterior chamber depth)
$\mathrm{AE}$
absoluter Vorhersagefehler (absolute error)
$A G$
Arbeitsgemeinschaft
AL
Achsenlängen
ApS
Anpartsselskab (dänische Rechtsform, vergleichbar mit
Gesellschaft mit beschränkter Haftung)
bzw. beziehungsweise
C C-Konstante
CCT zentrale Hornhautdicke (central corneal thickness)
CDVA korrigierter Fernvisus (corrected distance visual acuity)
CIVA fernkorrigierter Intermediärvisus (corrected intermediate visual acuity)
$\mathrm{cm} \quad$ Zentimeter
CNVA fernkorrigierter Nahvisus (corrected near visual acuity)
d. h. das heißt

$\begin{array}{ll}\text { dpt } & \text { Dioptrie } \\ \text { Dr. } & \text { Doktor }\end{array}$
ECCE extrakapsuläre Kataraktextraktion (extracapsular cataract extraction)
ELP effektive Linsenposition
et al und andere (et alii)
EVOF Emmetropia Verifying Optical-Formula
GmbH Gesellschaft mit beschränkter Haftung
H Hornhauthöhe
HicSoap Holladay IOL Consultant Software \& Surgical Outcomes
Assessment 


\begin{tabular}{ll} 
ICCE & intrakapsuläre Kataraktextraktion (intracapsular cataract extraction) \\
Inc. & incorporated \\
IOL & Intraokularlinsen \\
IQA & Interquartilsabstand \\
KI & Konfidenzintervall \\
kHz & Kilohertz \\
K-Wert & Hornhautbrechkraft, Keratometriewert \\
LLC & Gesellschaft mit beschränkter Haftung (Limited Liability Company) \\
logMAR & logarithm of the minimum angle of resolution \\
LP & Linsenstärke (lens power) \\
LT & Linsendicke (lens thickness) \\
m & Meter \\
m² & Quadratmeter \\
MAE & mittlerer absoluter Vorhersagefehler (mean absolute error) \\
MaxAE & maximaler absoluter Vorhersagefehler (maximum absolute error) \\
ME & mittlerer Vorhersagefehler (mean error) \\
MedAE & medianer absoluter Vorhersagefehler (median absolute error) \\
mg & Milligramm \\
ml & Milliliter \\
mm & Millimeter \\
mmHg & Millimeter Quecksilbersäule \\
$\mu J$ & Mikrojoule \\
$\mu m$ & Mikrometer \\
$\mathrm{n}$ & Brechungsindex \\
$\mathrm{nm}$ & Nanometer \\
OLCR & optische niedrig-kohärente Reflektometrie (optical low coherence \\
& reflectometry) \\
pACD & personalisierte Vorderkammertiefe (personalized anterior chamber \\
PCO & depth) \\
PE & Nachstar (posterior capsule opacification) \\
& Vorhersagefehler (prediction error) \\
\hline
\end{tabular}


$r \quad$ Hornhautradius

RBF radiale Basisfunktion

Ref Refraktion

RT Retinadicke (retinal thickness)

SD Standardabweichung (standard deviation)

SE $\quad$ sphärisches Äquivalent (spherical equivalent)

SF Surgeon Factor

SRK Sanders-Retzlaff-Kraff

SRK/T Sanders-Retzlaff-Kraff/ theoretical

u. a. unter anderem

UDVA unkorrigierter Fernvisus (uncorrected distance visual acuity)

UIVA unkorrigierter Intermediärvisus (uncorrected intermediate visual acuity)

ULIB User Group for Laser Interference Biometry

UNVA unkorrigierter Nahvisus (uncorrected near visual acuity)

US Vereinigte Staaten von Amerika (United States)

UV ultraviolett

$\mathrm{V} \quad$ Scheitelabstand einer Brille

VKD Vorderkammerdurchmesser

vs. versus

WTW Hornhautdurchmesser (white to white distance)

Z. B.

zum Beispiel 


\section{Einleitung}

So a future in which we can expect complication-free cataract surgery $99.9 \%$ of the time, with refractive precision essentially guaranteed and adjustable over time and with good presbyopia correction the standard, is a beautiful future to consider. Just Imagine $!^{A}$

(Randall J. Olson, 2018)

Randall J. Olson hat im Jahr 2018 der Kataraktchirurgie eine glorreiche Zukunft vorausgesagt. ${ }^{1}$ Die Behandlung des grauen Stars wird künftig eine immer größer werdende Rolle spielen, da mit steigender Lebenserwartung auch die Inzidenz der Katarakt ansteigen wird. Im Jahr 2010 war der graue Star mit 33,4\% die weltweit häufigste Ursache für Erblindung. 10,8 Millionen Patienten waren davon betroffen. $^{2}$

Schon in der römischen und arabischen Medizin wurden Kataraktoperationen durchgeführt. Mit einer Nadel wurde die getrübte Augenlinse nach unten gedrückt, sodass die optische Achse wieder frei war. Dieser als "Starstich" bezeichnete Eingriff führte zu schweren Infektionen bis hin zur Erblindung. ${ }^{3}$ Johann Sebastian Bach starb möglicherweise an den Komplikationen eines Starstichs. ${ }^{4}$ Die erste erfolgreiche Linsenentfernung wurde von Jacques Daviel im Jahr 1747 in Frankreich durchgeführt. ${ }^{5}$ Die erste Kunstlinse aus Polymethylmethacrylat wurde 1949 von Sir Nicholas Harold Lloyd Ridley, einem britischen Ophthalmologen, im St. Thomas Hospital in London implantiert. ${ }^{6}$ Die heute standardmäßig durchgeführte Technik der Kataraktoperation, die Phakoemulsifikation, wurde von Charles Kelman am New York Medical College begründet. ${ }^{7}$

Seit der Implantation der ersten Kunstlinse sind 70 Jahre vergangen und in Deutschland werden jährlich mehr als 800.000 Kataraktoperationen durchgeführt. ${ }^{8}$ Kein anderer Eingriff wird in der Medizin häufiger vorgenommen. ${ }^{9,10}$

\footnotetext{
A Übersetzung: Eine Zukunft, in der wir in 99,9\% der Fälle eine komplikationslose Kataraktoperation erwarten können, mit garantierter Präzision des refraktiven Ergebnisses und mit guter Presbyopiekorrektur als Standard, ist eine wundervolle Zukunft. Stellen Sie sich das mal vor!
} 
Umso wichtiger, dass die Entwicklung auf diesem Gebiet weiter vorangetrieben wird.

Nach einer Einführung in den Aufbau des Auges und die Katarakt werden die Grundlagen der Kataraktchirurgie dargelegt. Anschließend folgt die Erläuterung des Ablaufs und die Präsentation der Ergebnisse der vorliegenden Studie. Zum Schluss wird die Relevanz der Erkenntnisse erörtert und mögliche Fortschritte der nächsten Jahre werden diskutiert.

Um den Texffluss nicht zu stören, wurde bei Patienten und Berufsbezeichnungen stellvertretend die maskuline Form gewählt und auf die explizite Nennung der femininen Form verzichtet.

\section{1. $\quad$ Grundlagen}

\subsection{1. $\quad$ Aufbau des Auges}

Abbildung 1 zeigt den schematischen Aufbau des Auges in der Seitansicht.

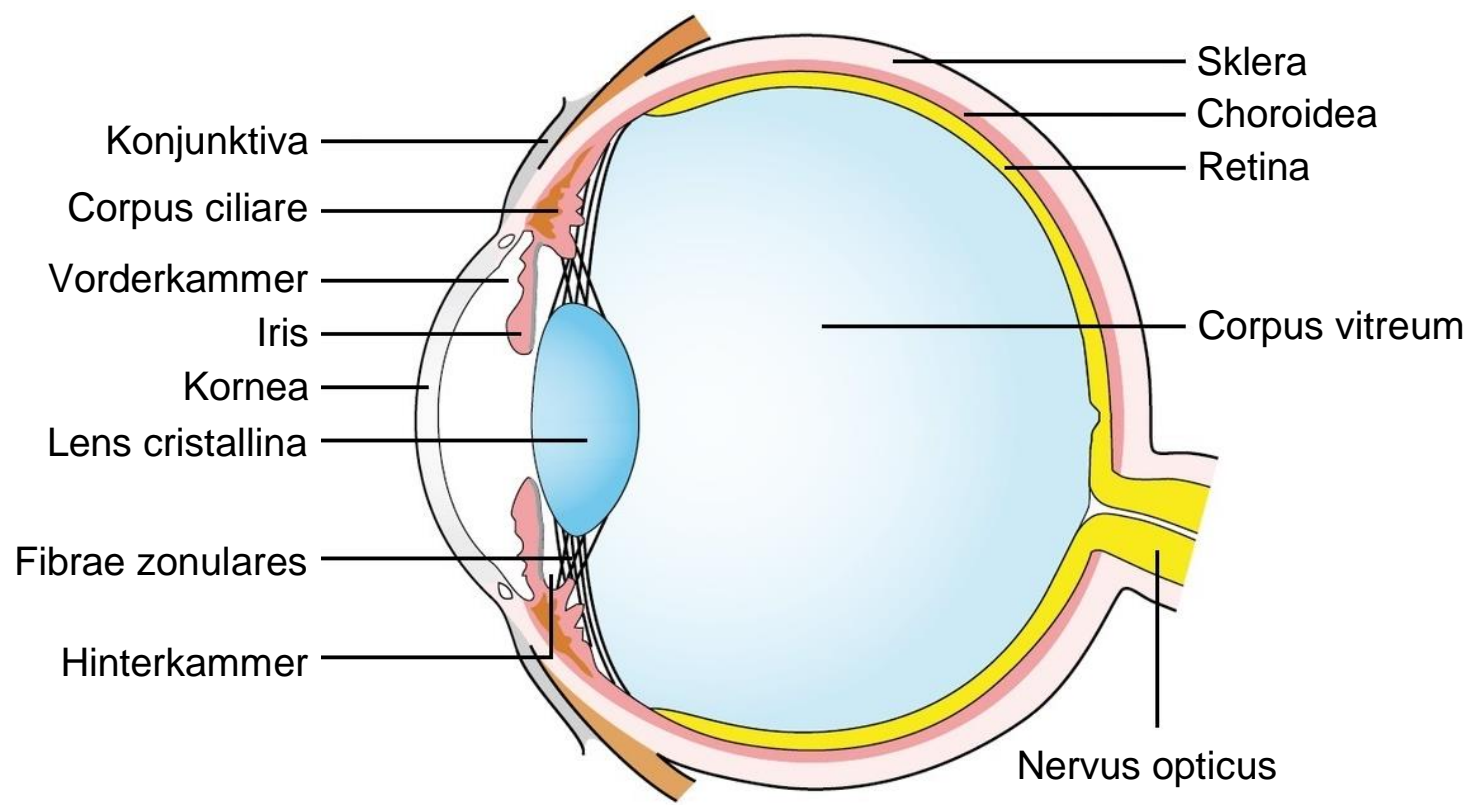

Abbildung 1: Seitansicht des Auges

(Abbildung verändert nach Huppelsberg und Walter ${ }^{11}$ ) 
Man kann das optische System des Auges als ein Linsensystem aus zwei Sammellinsen ansehen: der Hornhaut und der eigentlichen kristallinen Linse. Die Hornhaut hat mit etwa 43 Dioptrien (dpt) einen höheren Anteil an der Gesamtbrechkraft als die Linse. Bei Fernakkommodation beträgt deren Brechkraft etwa 15-20 dpt. ${ }^{12,13}$ Die hohe Brechkraft der Hornhaut kommt einerseits durch die Wölbung, andererseits durch den Unterschied der Brechungsindizes der Luft $(1,0)$ und der Hornhaut $(1,376)$ zustande..$^{9,14}$

Der vordere Augenabschnitt besteht aus der Konjunktiva (Bindehaut), der Kornea (Hornhaut), der Sklera (Lederhaut), der Iris (Regenbogenhaut), dem Corpus ciliare (Ziliarkörper), den Fibrae zonulares (Zonulafasern) und der Lens cristallina (Linse). Dazwischen befindet sich Kammerwasser in der Vorder- und Hinterkammer, welches vom Ziliarkörperepithel durch aktive Sekretion und Ultrafiltration des Bluts produziert wird. ${ }^{9}$

Der hintere Augenabschnitt beinhaltet das Corpus vitreum (Glaskörper), die Choroidea (Aderhaut) und die Retina (Netzhaut). Umgeben wird der hintere Teil des Auges ebenfalls von der Sklera. Der Nervus opticus (Sehnerv) enthält die Axone der retinalen Ganglienzellen. Er verlässt das Auge durch die Lamina cribrosa am hinteren Augenpol. ${ }^{9}$

Da es sich bei der Katarakt um eine Eintrübung der kristallinen Linse handelt, wird im Folgenden auf diese Struktur genauer eingegangen.

Die Augenlinse ist im Zentrum etwa $4 \mathrm{~mm}$ dick und misst im Durchmesser 8-10 mm. Sie ist eine bikonvexe Sammellinse, deren hintere Krümmung stärker ausgeprägt ist als die vordere. ${ }^{13}$ Der Brechungsindex der Linse beträgt zentral etwa 1,4 und peripher 1,36. Er unterscheidet sich damit von den Brechungsindizes des Kammerwassers $(1,3374)$ und des Glaskörpers $(1,336)$, wodurch die Linse ihre Fähigkeit zur Lichtbrechung erhält. ${ }^{12,14}$ Im Alter nimmt der Brechungsindex der Linse vermutlich aufgrund der Akkumulation unlöslicher Proteinpartikel zu. ${ }^{12,15}$

Die Linse besteht aus Linsenepithel, Rinde und Kern (siehe Abbildung 2). Umgeben ist sie von der Linsenkapsel, einer 5-20 $\mu \mathrm{m}$ dicken Basallamina aus Kollagen Typ IV. ${ }^{12}$ 


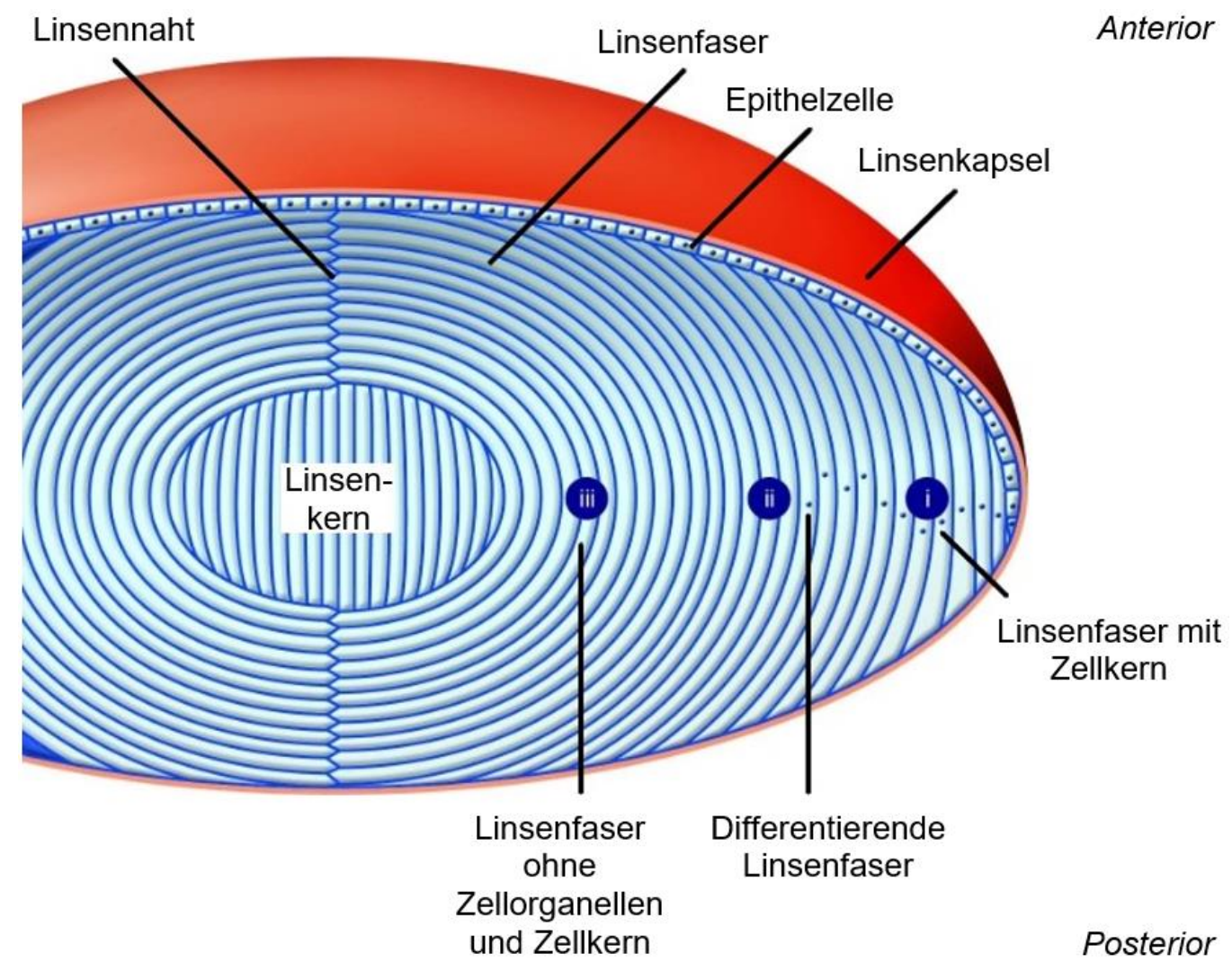

Abbildung 2: Histologischer Aufbau der Linse

(Abbildung verändert nach Song et al' ${ }^{16}$ )

Kern und Rinde bestehen zu 35-40\% aus Wasser und zu 60-65\% aus Linsenfasern. ${ }^{9,17}$ Das einschichtige Linsenepithel befindet sich nur an der Innenseite der vorderen Linsenkapsel und des -äquators. ${ }^{9}$ Die zylindrischen Epithelzellen am Äquator bilden durch Zellteilung lebenslang neue, sekundäre Linsenfasern, die sich den schon embryonal vorhandenen, primären Fasern von peripher anlagern. Die Linsenrinde enthält somit jüngere Linsenfasern als der Kern. ${ }^{12,17}$ Linsenfasern sind vitale Zellen, die im Laufe der Zeit den Zellkern und die Zellorganellen verlieren. Sie beinhalten Linsenfaserproteine, vor allem Kristalline, die wegen ihrer dichten Struktur für die Transparenz verantwortlich sind. Um die Proteine vor oxidativem Stress, insbesondere durch kurzwelliges Licht, zu schützen, enthält die Linse antioxidative Ascorbinsäure, Superoxid-Dismutase, Katalase und Glutathion-Peroxidase. Oxidativer Stress fördert die Denaturierung der Proteinstruktur. ${ }^{17}$

Die Zonulafasern halten die Linse zwischen der Iris und dem Glaskörperraum. Sie gehen von der vorderen und hinteren Kapsel aus und setzen am Ziliarkörper 
an. Um Gegenstände in der Nähe scharf auf der Netzhaut abbilden zu können, muss das Auge zur Akkommodation fähig sein. Bei der Akkommodation kontrahiert sich der ringförmige Ziliarmuskel, wodurch der Zug der Zonulafasern auf die Linse nachlässt und diese sich durch ihre Eigenelastizität stärker wölbt. Dadurch nimmt ihre Brechkraft zu. ${ }^{12,13}$ Aufgrund der Abgabe von Wasser werden die Linsenfasern im Laufe des Lebens allmählich dünner und verdichten sich. Die zunehmende Verhärtung und die Aggregation von Proteinen führt dazu, dass die Elastizität der kristallinen Linse im Alter abnimmt. ${ }^{12,18}$ Sie verliert dadurch ihre Fähigkeit zur Akkommodation, was die Entwicklung einer Presbyopie (Altersweitsichtigkeit) bedingt. ${ }^{12,19}$

Da die Linse keine Gefäße enthält, muss sie per Diffusion vom Kammerwasser ernährt werden. ${ }^{17}$ Die negativ geladenen Kristalline bewirken, dass sich in der Linse viele Kationen befinden. Durch das Linsenepithel wird Natrium in das Kammerwasser gepumpt, wohingegen Kalium in die Linse eingelagert wird. Die Kalziumkonzentration sollte durch aktiven Transport sehr gering bleiben. Bei Störungen der Transportmechanismen oder der Elektrolytzusammensetzung des Kammerwassers kann es zu Linsentrübungen kommen. ${ }^{9}$

\subsubsection{Katarakt}

\subsubsection{Pathophysiologie und Risikofaktoren}

Als Katarakt oder grauen Star bezeichnet man Eintrübungen der kristallinen Linse, durch die das Sehvermögen eingeschränkt ist. ${ }^{18}$ Das Wort „Katarakt“ stammt vom Griechischen „katarrákti“ und bedeutet „Wasserfall“. Früher nahm man an, dass die Trübung durch geronnene Flüssigkeit zustande kommt, die sich hinter der Pupille wasserfallartig ergießt. Die Bezeichnung "grauer Star“ wurde aufgrund des starren Blicks gewählt, durch den die Betroffenen bei vollständiger Erblindung auffallen. ${ }^{9}$

Die Trübung der Augenlinse ist ein physiologischer, multifaktorieller Prozess. Veränderungen sind etwa ab dem 50. Lebensjahr sichtbar. Im Alter kommt es zu einer Beeinflussung der Interaktion zwischen den Linsenproteinen. Durch die Denaturierung wird die fest angeordnete Proteinstruktur und somit die Streuung 
des Lichts gestört. ${ }^{18,20}$ Der Wasserverlust trägt zusätzlich dazu bei, dass die Linse ihre Transparenz verliert. ${ }^{9}$

Neben steigendem Alter als Haupteinflussfaktor werden je nach Form verschiedene Auslöser für die Kataraktentwicklung angenommen. Als allgemeine Risikofaktoren gelten Adipositas, Diabetes mellitus, Hypertonie, Rauchen, starker Alkohol- oder Drogenkonsum sowie die regelmäßige Einnahme von Steroiden. ${ }^{18,21,22}$ Auch das weibliche Geschlecht wird als Risikofaktor, insbesondere für Rindenkatarakte, diskutiert. ${ }^{21,22}$ Das Auftreten des grauen Stars wird vor allem durch die Einnahme von Östrogenen begünstigt. ${ }^{21}$ Ein hohes Bildungsniveau reduziert das Risiko der Kataraktentwicklung, was sich vermutlich durch einen gesünderen Lebensstil erklären lässt. ${ }^{21,22}$

Auslöser für eine Cataracta complicata sind $u$. a. eine Uveitis, ein Glaukom, vitreo-retinale Erkrankungen oder eine extreme Myopie. Letztere führt oft zu einer Cataracta subcapsularis posterior. Auch nach einer Vitrektomie oder einer Glaukomoperation kann eine Katarakt auftreten. ${ }^{9}$ Die Strahlenkatarakt wird durch Infrarot-, Ultraviolett- (UV) oder Röntgenstrahlung verursacht. ${ }^{18}$ Eine Cataracta traumatica kann durch Verletzungen hervorgerufen werden. ${ }^{9}$

Der graue Star ist in den meisten Fällen eine Alterserkrankung (Cataracta senilis). Nur selten sind auch Babys und Kleinkinder betroffen (Cataracta congenita). ${ }^{18}$ In diesem Fall liegen meist Mutationen in verschiedenen Genen vor, die beispielsweise für Linsenfaserproteine oder Wachstumsfaktoren kodieren. Bestimmte Genvariationen führen außerdem zu einer erhöhten Anfälligkeit für eine altersbedingte Linsentrübung. ${ }^{23}$

Da die Linsenveränderungen im Alter bei jedem Menschen auftreten, gibt es keine Methode, um dem grauen Star vorzubeugen. Es hat sich jedoch erwiesen, dass das Tragen von Sonnenbrillen zum Schutz vor UV-Strahlung, eine vitaminreiche Ernährung und der Verzehr von Lebensmitteln mit vielen Omega-3Fettsäuren und Antioxidantien das Risiko senken können. 21,24,25 Eine 2015 publizierte Arbeit aus China hat in Labor- und Tierexperimenten festgestellt, dass Lanosterol die Proteinaggregation in der Linse hemmen kann. ${ }^{26}$ Versuche am menschlichen Auge stehen noch aus. 


\subsubsection{Morphologische Einteilung}

Rindenstare (Cataracta corticalis) machen die Hälfte aller Alterskatarakte aus. Sie können zentral oder peripher auftreten und beginnen als graue radiäre Keile, Speichen oder Wasserspalten. Die Patienten beklagen sich über eine erhöhte Blendempfindlichkeit und einen verschwommenen Seheindruck. Diese Form der Katarakt schreitet schneller voran als die Kernkatarakt. ${ }^{9,18}$

Die subkapsuläre hintere Rindentrübung (Cataracta subcapsularis posterior) ist die zweithäufigste Form der Alterskatarakte. Sie führt rasch zu Einschränkungen des Sehvermögens, da sie in der optischen Achse beginnt und sich in die Peripherie ausbreitet. Diese Form tritt häufiger bei jungen Patienten auf sowie unter langfristiger Kortisontherapie oder bei Alkoholabusus. Auffällig ist neben einer erhöhten Blendempfindlichkeit eine erhebliche Einschränkung des Nahvisus. Grund dafür ist die Miosis bei Nahakkommodation. Bei Mydriasis, z. B. in der Dämmerung, verbessert sich der Visus. ${ }^{9,18}$

Die Kernkatarakt (Cataracta nuclearis) tritt gehäuft bei hoher Myopie auf. Die Linse fällt durch eine bräunlich-gelbe Färbung auf. Der Brechungsindex und somit die Brechkraft nehmen zu, wodurch es zu einer Brechungsmyopie oder Reduktion einer vorhandenen Hyperopie kommt. Dadurch bleibt das Nahsehvermögen relativ lange unbeeinträchtigt. Im späteren Stadium färbt sich die Linse dunkel und wird undurchsichtig. ${ }^{9,18}$

Beim meist hereditären Schichtstar (Cataracta zonularis) befindet sich die Trübung in einer einzigen Linsenfaserschicht. Wenn diese äquatorial angeordnet ist, bezeichnet man sie als „Reiterchen“. ${ }^{9}$

Eine weitere vererbte Form ist der Kranzstar (Cataracta coronaria bzw. coerulea). Die Trübungen befinden sich kranzförmig am Linsenäquator. Die Linse erscheint bläulich. ${ }^{9}$ 


\subsubsection{Klinik}

Die Erkrankung verläuft schleichend und schmerzlos. Sie führt zu keiner Rötung der Augen. Die visuelle Einschränkung durch die Trübung kann sich unterschiedlich äußern und wird den Patienten meist erst relativ spät bewusst. ${ }^{18}$ Häufig haben Patienten Probleme beim Lesen, weil durch die Miosis bei Nahakkommodation nur der zentrale Anteil der Linse genutzt werden kann, welcher meist stärker eingetrübt ist. ${ }^{9}$ Bei geringem Kontrast ist das Sehvermögen stark reduziert. Oft fällt es Betroffenen schwer, im Dämmerlicht zu sehen oder sie haben den Eindruck, als hinge ein grauer Schleier vor ihren Augen. Durch die trübe, gelbliche Linse erscheinen Farben verblasst oder Gegenstände braungelb verfärbt. Der Blauanteil des Lichts wird besonders herausgefiltert. Die Patienten klagen über Verschwommensehen und Blendung, da das Licht durch die Trübung unregelmäßig und diffus gestreut wird. Ein weiteres Symptom sind Halos, d. h. Lichthöfe, die bei Nacht um punktförmige Lichtquellen herum zu sehen sind.9,18 Zur monokularen Diplopie kommt es, wenn durch die unterschiedlichen Brechungsindizes der Kerntrübungen oder durch speichenförmige Trübungen zwei Brennpunkte entstehen. ${ }^{9}$

\subsubsection{Therapie}

Eine kurative Therapie des grauen Stars ist nur durch eine Operation möglich. Da die Erkrankung auf einer irreversiblen Linseneintrübung beruht, besteht keine Möglichkeit, eine Katarakt konservativ zu heilen. Mydriatika können den Visus bei bestehender Cataracta subcapsularis posterior verbessern. Der Patient sollte darauf hingewiesen werden, dass die Progredienz der Erkrankung durch die Verminderung von Risikofaktoren hinausgezögert werden kann, z. B. durch den Rauchverzicht oder die adäquate Therapie von Begleiterkrankungen. ${ }^{18}$ Bei einer diagnostizierten Katarakt finden jährlich augenärztliche Kontrolluntersuchungen statt. Dieses Intervall wird bei raschem Fortschreiten oder stark myopen Patienten auf drei bis sechs Monate verkürzt. ${ }^{27}$ 
Die Indikationsstellung zur Operation umfasst drei Hauptaspekte.

Der erste Punkt ist eine Verschlechterung der Sehleistung, die den Patienten stark einschränkt oder sogar gefährdet, z. B. beim Autofahren. Ab einer korrigierten Sehschärfe von 0,6 sollte man eine Operation empfehlen. ${ }^{9}$ Weitere Indikationen sind eine starke Anisometropie, Entzündungen, ein sekundäres Glaukom oder eine ungenügende Beurteilung des Augenhintergrundes aufgrund der Linsentrübung. ${ }^{18} \mathrm{Im}$ Rahmen vitreo-retinaler Eingriffe sowie bei einer Linsenluxation oder Linsenquellung nach einem Trauma soll dringend zur einer Kataraktoperation geraten werden. ${ }^{27}$

Zum Zweiten sollte man sicherstellen, dass der Patient nach der Operation aller Wahrscheinlichkeit nach wieder besser sehen kann. Eine präoperative Sehschärfemessung mit einem Retinometer kann eine Einschätzung bezüglich des postoperativen Sehvermögens geben. ${ }^{9}$

Der dritte Aspekt besteht im Abwägen des Kosten-Nutzen-Risikos unter Betrachtung des individuellen Operationsrisikos. ${ }^{9}$

Die Operation besteht aus der Extraktion der eingetrübten Linse und der anschließenden Implantation einer künstlichen Intraokularlinse (IOL). Hauptziel des operativen Eingriffs ist die bestmögliche Wiederherstellung der Sehkraft des Patienten und damit verbunden eine verbesserte Lebensqualität. Außerdem können nach der Operation Veränderungen des Augenhintergrundes, z. B. eine Makuladegeneration oder eine diabetische Retinopathie, erkannt und behandelt werden. ${ }^{27}$ 


\subsection{Durchführung der Kataraktoperation}

\subsubsection{Anästhesie}

Der Eingriff findet bevorzugt ambulant statt, sofern keine medizinischen oder sozialen Kontraindikationen bestehen. ${ }^{27}$

Im Regelfall wird die Kataraktoperation in Lokalanästhesie durchgeführt. Mit Tropfanästhetika wie Tetracain wird nur die Augenoberfläche betäubt. Bei diesem Verfahren kann der Patient das Auge noch bewegen, was während der Operation dringend vermieden werden sollte. Bei der Retrobulbäranästhesie wird ein Lokalanästhetikum, z. B Lidocain oder Bupivacain, in den Retrobulbärraum gespritzt und dadurch eine Leitungsanästhesie der motorischen und sensiblen Nervenfasern erreicht. Im Gegensatz dazu wird bei einer Parabulbäranästhesie das Lokalanästhetikum außerhalb des Muskeltrichters injiziert, wodurch die Verletzungsgefahr von Nerven und Gefäßen reduziert werden soll. Bei den beiden letztgenannten Formen ist der Patient wach, kann das Auge aber nicht bewegen. ${ }^{9}$

Nur in Ausnahmefällen ist eine Allgemeinanästhesie nötig. ${ }^{9}$

\subsubsection{Hornhautinzision}

Die Hornhautinzision wird meist an der temporalen Seite entweder sklerokorneal, d. h. am Übergang von Kornea zu Sklera, oder als Clear-Cornea-Inzision durch die Hornhaut durchgeführt. Die Größe der Hornhautinzision beeinflusst den induzierten postoperativen Astigmatismus. Es besteht daher das Bestreben, die Inzisionen so klein wie möglich zu halten. Gleichzeitig muss allerdings ausreichend Platz für das möglichst atraumatische Einführen der Kunstlinse vorhanden sein. Bei zu kleinen Inzisionen kommt es zu unerwünschten Einrissen an den Rändern der Inzisionsstelle. ${ }^{28}$

Die Mikroinzisionschirurgie nutzt Schnitte von weniger als $2 \mathrm{~mm}$. Vorteile sind u. a. eine geringere Störung der kornealen Integrität und Stabilität und eine schnellere visuelle Rehabilitation. ${ }^{28}$ Bei der Verwendung von kleineren Phakonadeln ist jedoch deren Leistung herabgesetzt, was das Zerkleinern und 
Absaugen der Linse erschwert. Außerdem muss ausreichend Flüssigkeit nachströmen können, um die Vorderkammer stabil zu halten. Da auch die Linsen anders aufgebaut sein müssen, was zu erhöhten postoperativen Nachstarraten und Refraktionsverschiebungen führt, hat sich diese Technik nicht durchgesetzt. $^{29}$

Die Größe der Hornhautinzisionen beträgt derzeit meist etwa 2,75 mm und ist abhängig von der Phakotechnologie, der Operationstechnik und dem Design der implantierten Linse. Bei präoperativ vorhandenem Astigmatismus kann die Schnittführung so gewählt werden, dass die Hornhautverkrümmung reduziert wird. ${ }^{28}$

Eine andere, sehr präzise Möglichkeit, Hornhautschnitte zu erzeugen, ist die Nutzung eines Femtosekundenlasers. ${ }^{30}$

\subsubsection{Entfernung der getrübten Linse}

Es gibt im Wesentlichen drei Möglichkeiten, die körpereigene Linse zu entfernen: die intrakapsuläre (intracapsular cataract extraction, ICCE) und extrakapsuläre Linsenextraktion (extracapsular cataract extraction, ECCE) sowie die Phakoemulsifikation.

Die ICCE wird heutzutage nur noch in Ausnahmefällen durchgeführt, wenn z. B. die Zonulafasern abgerissen sind. Die Linse wird zusammen mit der Kapsel entfernt. Die Kunstlinse wird dann irisfixiert in die Vorderkammer oder transskleral fixiert in die Hinterkammer eingesetzt. Ein Nachstar ist bei der ICCE ausgeschlossen, da die Kapsel komplett entfernt wird. ${ }^{9}$

Bei der ECCE wird lediglich die vordere Kapsel kreisförmig eröffnet. Die hintere Kapsel bleibt intakt. Die Kapsulorhexis kann manuell mit einer Pinzette oder Nadel durchgeführt werden. ${ }^{29}$ Eine präzisere Möglichkeit ist die Kapsulotomie mittels Femtosekundenlaser, die seit etwa zehn Jahren zur Verfügung steht. Die Linsenkapsel wird per Hydrodissektion vom Linsenkörper getrennt, um die Entfernung der Linse zu vereinfachen und Schäden der hinteren Linsenkapsel zu verhindern. ${ }^{9}$ 
Aufgrund der hohen Kosten für ein Phakoemulsifikationsgerät wird in ökonomisch benachteiligten Ländern bevorzugt die ECCE mit Expression des Kerns und Absaugen der Rinde durchgeführt. Da der Kern als Ganzes entfernt wird, ist ein etwa 9-12 mm großer Schnitt nötig. Entscheidende Nachteile sind der induzierte Astigmatismus und die verlängerte Rehabilitationsphase. ${ }^{31}$ Außerdem muss die Inzision gegebenenfalls genäht werden. Die ECCE mit Kernextraktion wird daher in Deutschland selten durchgeführt. Sie ist eine Möglichkeit bei besonders harten Linsenkernen, da bei der Phakoemulsifikation zu viel Energie nötig wäre, wodurch das Hornhautendothel geschädigt werden könnte. ${ }^{9}$

Die heutzutage am häufigsten durchgeführte Methode ist die Phakoemulsifikation, eine minimalinvasive Variante der ECCE. Linsenrinde und -kern werden dabei von der Phakospitze angezogen, mittels Ultraschall zerkleinert und anschließend abgesaugt. Der nur etwa $2,75 \mathrm{~mm}$ große Schnitt verschließt sich nach der Operation ventilartig. ${ }^{9}$

Die Linse kann bei der Phakoemulsifikation auch vorab mit einem Femtosekundenlaser fragmentiert werden. ${ }^{29}$ Es ist dann eine geringere Ultraschallleistung zum Zerkleinern der Linse erforderlich. In einigen Fällen ist sogar keine weitere Ultraschallenergie nötig. ${ }^{32,33}$ Dadurch wird das umgebende Gewebe geschont.

\subsubsection{Implantation der Kunstlinse}

Nach der Extraktion der getrübten Linse kann die Kunstlinse mit Hilfe eines Injektors platziert werden. Standardmäßig werden faltbare Hinterkammerlinsen verwendet, die in den verbliebenen Kapselsack implantiert werden. Vor allem bei Linsen mit speziellen Optiken ist eine exakte Platzierung essentiell, um optische Aberrationen und eine damit verbundene Sehbeeinträchtigung zu vermeiden. $\mathrm{Zu}$ diesen Linsen zählen beispielsweise torische oder multifokale IOL. ${ }^{34}$

Für den Fall, dass Anomalien der Zonulafasern vorliegen oder der Kapselsack gerissen ist, ist die normale Positionierung einer Hinterkammerlinse im Kapselsack nicht möglich. Eine Möglichkeit ist dann die Implantation einer Vorderkammerlinse. Alternativ kann eine Hinterkammerlinse im Ziliarsulkus platziert 
werden. ${ }^{35}$ Dabei ist es wichtig, die Haptik an der Iris oder Sklera zu vernähen, um eine korrekte Position zu gewährleisten. ${ }^{36}$ Trotz Fixierung besteht allerdings die Gefahr der Dezentrierung oder Verkippung. Außerdem können die Nähte reißen oder sich lockern. Es muss beachtet werden, dass sich die Linse im Ziliarsulkus weiter vorne befindet. Die berechnete IOL-Stärke für eine intrakapsuläre Implantation muss daher reduziert werden. ${ }^{37}$

Linsen mit Plattenhaptiken oder akkommodative Linsen müssen unbedingt an der Hinterwand des Kapselsacks fixiert werden. Auch einteilige Acryllinsen sollten nicht im Ziliarsulkus positioniert werden, da sich andernfalls die Zahl der Komplikationen drastisch erhöht. ${ }^{38}$

\subsection{5. $\quad$ Risiken der Operation}

Insgesamt ist die Kataraktoperation ein sicherer Eingriff. Die Rate schwerwiegender Komplikationen ist mit etwa $1 \%$ sehr gering. ${ }^{18}$

Intraoperativ sind Blutungen, ein Verlust des Glaskörpers, ein Abfall des intraokularen Drucks sowie Verletzungen der Hornhaut, der Iris, des Ziliarkörpers mit den Zonulafasern oder des Kapselsacks möglich. ${ }^{18}$

Postoperativ können eine Wundheilungsstörung, ein Hornhautödem oder ein zystisches Makulaödem auftreten. Ein erhöhter Augeninnendruck in der frühen postoperativen Phase kann zu einer Dekompensation des Hornhautendothels oder einem Hornhautödem führen. ${ }^{18}$

Wie bei jedem operativen Eingriff sind Infektionen, z. B. eine Iritis, nicht ausgeschlossen. Eine sehr seltene, aber schwerwiegende Komplikation ist die infektiöse Endophthalmitis. ${ }^{18}$ Ein erhöhtes Risiko besteht bei fortgeschrittenem Alter des Patienten, einer größeren Inzision und intraoperativen Komplikationen. ${ }^{18,39}$ Eine intrakamerale Injektion von Cefuroxim oder Moxifloxacin am Ende der Operation reduziert die Wahrscheinlichkeit für das Auftreten dieser Komplikation. ${ }^{40}$ Die auslösenden Bakterien entstammen meist der periokularen Haut, wodurch der Hautdesinfektion mit 10\%iger Povidon-lod-Lösung und der Applikation von 5\%iger Lösung in den Konjunktivalsack besondere Bedeutungen zukommen. ${ }^{41,42}$ Wichtig ist auch ein dichter Verschluss aller Inzisionen. ${ }^{39}$ Die 
Gefahr der Erblindung als Folge einer Endophthalmitis ist sehr hoch. ${ }^{9}$ Von der Endophthalmitis muss das Toxic Anterior Segment Syndrome abgegrenzt werden, das meist zwölf bis 48 Stunden nach der Operation auftritt. Es ist gekennzeichnet durch eine akute Sehverschlechterung, ein Hornhautödem und einen starken Reizzustand der Vorderkammer. Durch die nicht-infektiöse, sterile Entzündung kommt es zu einer toxischen Schädigung von intraokularem Gewebe des Vorderabschnitts, was in einer langfristigen Dekompensation des Hornhautendothels resultieren kann. ${ }^{18}$

Grundsätzlich ist nach refraktiven Eingriffen eine verringerte Kontrastsensitivität möglich, was zu einer eingeschränkten Sehfähigkeit in der Dämmerung und bei Nacht führt. Es können außerdem Glares, d. h. Glanzeffekte, und Halos auftreten. ${ }^{18,43}$

Die häufigste Folgeerscheinung nach einer Kataraktoperation ist der Nachstar (posterior capsule opacification, PCO). In Abhängigkeit vom Operationsverlauf, der Art der implantierten Linse und weiteren Erkrankungen des Auges tritt diese Eintrübung der hinteren Linsenkapsel etwa bei einem Drittel der Patienten in den ersten fünf Jahren auf. ${ }^{44}$ Der fibrotische Nachstar entsteht durch eine Fibrosierung der Hinterkapsel. Der regeneratorische Nachstar kommt durch verbliebene Zellen der körpereigenen Linse am Linsenäquator zustande, die proliferieren und metaplastisch werden. ${ }^{9}$ Indikationen zur Behandlung der PCO sind Beeinträchtigungen des Sehvermögens und eine eingeschränkte Fundusbeurteilung. ${ }^{18}$ Die Standardtherapie ist die Laserkapsulotomie. Mittels eines Neodym-dotierten Yttrium-Aluminium-Granat-Lasers werden kleine Löcher in die Hinterkapsel geschossen. Der Eingriff wird in Mydriasis am Spaltlampenmikroskop durchgeführt. Mögliche Risiken sind Netzhautablösungen, zystoide Makulaödeme, eine kurzzeitige Erhöhung des Augeninnendrucks oder eine Dislokation der IOL. Postoperativ werden gegebenenfalls Medikamente zur Senkung des Augeninnendrucks oder zur Entzündungshemmung verabreicht. ${ }^{45}$ Die PCO-Rate kann durch die Verwendung von Linsen mit scharfen hinteren Optikkanten verringert werden. ${ }^{44,46}$ Eine Möglichkeit, Nachstare zu vermeiden, ist die hintere Kapsulorhexis. ${ }^{47}$ 
Weitere postoperative Probleme sind Dezentrierung und Dislokation der implantierten Linse. Bei torischen Linsen besteht die Gefahr der Rotation und somit der Verstärkung des kornealen Astigmatismus. ${ }^{9}$

\subsection{Einflüsse auf das Ergebnis der Kataraktchirurgie}

Verschiedene Faktoren haben einen Einfluss auf das endgültige Ergebnis der Operation. Wichtige Rollen spielen die Auswahl einer geeigneten Linse und deren Eigenschaften, die Auswahl des Messgeräts und die Genauigkeit der Vermessung, die Verwendung einer geeigneten Formel zur Berechnung der IOLStärke und die Optimierung der Linsenkonstanten sowie die Operationstechnik und die Erfahrung des Chirurgen. ${ }^{48-50}$ Bei Muslimen sollte beispielsweise darauf geachtet werden, dass die präoperativen Messungen nicht während des Ramadan stattfinden, da in dieser Zeit die Tiefe der Vorderkammer um durchschnittlich $1 \mathrm{~mm}$ zunimmt, während die axiale Länge des Auges dementsprechend abnimmt. ${ }^{51}$

\subsubsection{Arten von Intraokularlinsen}

\subsubsection{Linsengröße, -material und -haptik}

Kunstlinsen sind in verschiedenen Größen erhältlich. Große Linsen mit einem Durchmesser von über $6 \mathrm{~mm}$ sind weniger abhängig von einer korrekten Zentrierung, führen zu geringeren photischen Phänomenen bei Mydriasis und zeigen ein geringeres PCO-Risiko. ${ }^{52}$ Ein Nachteil großer Linsen ist, dass eine größere Inzision nötig ist, um sie einzusetzen.

Bezüglich des Linsenmaterials unterscheidet man harte und weiche Linsen. Die früher verwendeten harten Linsen aus Polymethylmethacrylat, wie sie z. B. Sir Harold Ridley eingesetzt hat, werden zunehmend von weichen Linsen aus hydrophilem oder hydrophobem Acryl, Silikon oder einem Kollagen/ Hydroxyethylmethacrylat-Copolymer abgelöst. Der entscheidende Vorteil der weichen Linsen ist, dass sie faltbar sind und somit eine kleinere Inzision nötig ist, um sie einzusetzen. ${ }^{18} \mathrm{Um}$ das Risiko einer Endophthalmitis zu vermindern, werden 
Injektoren zum Einsetzen der Linse in den Kapselsack verwendet. Dadurch wird ein Kontakt der Kunstlinse mit umgebenden Strukturen vermieden. ${ }^{53}$

Die natürliche Linse verfärbt sich physiologischerweise im Alter gelblich, wodurch der Blauanteil des einfallenden Lichts herausgefiltert wird. Würde man sie durch eine klare Kunstlinse ersetzen, führte das zu einem größeren Lichteinfall und somit zu einem höheren Risiko für eine Makuladegeneration. Aus diesem Grund haben die meisten Linsen einen Blaulichtfilter. ${ }^{13,52}$ Blaulichtfilter-IOL tragen außerdem zu einem besseren Kontrastsehen bei. ${ }^{54}$

Eine Kunstlinse besteht aus einer optischen Linse im Zentrum und einer peripheren Haptik, die die IOL im Linsenäquator fixiert. Am häufigsten werden elastische Bügel, d. h. C-Haptiken, oder Plattenhaptiken verwendet. ${ }^{9}$ Neben einteiligen Linsen gibt es auch mehrteilige Linsen, welche aus verschiedenen Materialien aufgebaut sind.

\subsubsection{Optische Eigenschaften der verschiedenen Linsenarten}

Aberrationen entstehen, wenn Lichtstrahlen am Rand eines optischen Elements, wie z. B. der Kornea oder der Augenlinse, zu stark gebrochen werden. Bei erweiterter Pupille nimmt die effektive Brechkraft der Hornhaut durch positive sphärische Aberrationen zu. ${ }^{55}$ Ein Lichtpunkt wird dann unscharf auf der Retina abgebildet. ${ }^{52}$ Bei Jugendlichen nimmt die Linsenkrümmung Richtung Peripherie ab. Durch die resultierende negative sphärische Aberration kann die positive Aberration der Hornhaut ausgeglichen werden. Im Alter geht diese Kompensationsfähigkeit jedoch verloren. ${ }^{29} \mathrm{Im}$ Vergleich zu aberrationsneutralen sphärischen IOL können aberrationskorrigierende asphärische IOL durch eine nach peripher abnehmende Krümmung die sphärischen Aberrationen des Gesamtsystems vermindern. Dadurch verbessern sich die Kontrastsensitivität und die Sehqualität, wohingegen sich die Tiefenschärfe verschlechtert. ${ }^{56}$ Asphärische Linsenkomponenten sind daher vor allem bei Multifokallinsen sinnvoll. ${ }^{54,57}$ Eine Verbesserung der Sehleistung ist jedoch umstritten. ${ }^{56,58}$ Die postoperativen Ergebnisse können durch eine individuelle Anpassung der Asphärizität der IOL an die Asphärizität der Kornea verbessert werden. ${ }^{29,59,60}$ Die vom Hersteller angegebene Linsenstärke entspricht der paraxialen, d. h. der 
zentralen Brechkraft. Ein asphärischer Anteil führt zu negativen sphärischen Aberrationen und somit zu einer Reduktion der effektiven Brechkraft. Je asphärischer die Linse, desto höher muss die Linsenstärke gewählt werden, um die gleiche effektive Brechkraft wie mit einer aberrationsneutralen Linse zu erreichen. Prinzipiell sind für die IOL-Kalkulation von asphärischen Linsen exakte Raytracing-Formeln besser geeignet (siehe Seite 38 ). ${ }^{55}$

Je nach Anzahl der Brennpunkte unterscheidet man monofokale und multifokale Linsen. Monofokallinsen generieren nur einen Brennpunkt, der meist in der Ferne liegt. Der Patient benötigt dann für den Nahbereich eine Sehhilfe. Multifokallinsen bündeln Licht in mehreren Brennpunkten, wodurch Objekte in unterschiedlichen Abständen scharf auf der Retina abgebildet werden können. Sie ermöglichen daher im Idealfall eine Brillenunabhängigkeit des Patienten. Das Gehirn des Patienten muss lernen, die Aufmerksamkeit jeweils auf das schärfste Bild zu lenken bzw. die Abbildungen der nicht genutzten Brennpunkte auszublenden. ${ }^{29}$ Das nicht-fokussierte Bild wird als "out of focus image" bezeichnet und von Patienten aufgrund der geringen Lichtintensität normalerweise nicht als störend empfunden. ${ }^{59}$ Bifokale Linsen haben zwei Brennpunkte, einen im Nahbereich bei etwa $40 \mathrm{~cm}$ und einen in der Ferne. Trifokale Linsen generieren einen weiteren Brennpunkt im Zwischenbereich. Die Einführung von Multifokallinsen hat die Kataraktchirurgie revolutioniert. Die neueste Entwicklung ist eine quadrifokale Linse der Firma Alcon, die sich funktionell wie eine trifokale verhält (vergleiche 5.3, Seite 45). Der Brennpunkt im Intermediärbereich liegt im Gegensatz zu anderen Trifokallinsen bei $60 \mathrm{~cm}$ statt bei $80 \mathrm{~cm}$. Da dieser Abstand etwa einer Armlänge entspricht, ist die quadrifokale AcrySof IQ PanOptix TFNT00 (Alcon Laboratories Inc., Fort Worth, Texas, US) für die Arbeit im Nah- und Zwischenbereich optimal geeignet. ${ }^{61,62}$ Sie wurde im Juli 2015 von Professor Dr. Thomas Kohnen weltweit erstmalig eingesetzt. Nachteile multifokaler Linsen sind das gehäufte Auftreten von photischen Phänomenen, wie Glares und Halos, weshalb sie nicht für Personen geeignet sind, die nachts Autofahren müssen. ${ }^{43,62}$ Die Angaben bezüglich Halos nach einer Implantation der PanOptix schwanken laut einer Metaanalyse zwischen weniger als $1 \%$ und $89 \%$, wobei die meisten Patienten sich dadurch nicht in ihrer Lebensqualität beeinträchtigt fühlen. ${ }^{63} \mathrm{Da}$ 
die Kontrastsensitivität geringer ist als mit monofokalen Linsen, sollte für Multifokallinsen ein asphärisches Design gewählt werden. ${ }^{54,57}$

Multifokallinsen haben entweder eine refraktive oder eine diffraktive Optik. Refraktion bezeichnet die Brechung von Lichtstrahlen an einer Grenzfläche zwischen zwei Medien mit unterschiedlichen Brechungsindizes. ${ }^{19}$ Ein Teil des Lichts wird beim Übertritt vom einen in das andere Medium reflektiert. Die refraktiven Linsen besitzen auf der Vorderfläche zwei oder mehr konzentrische Zonen unterschiedlicher Refraktion. ${ }^{19,64}$ Bei Miosis wird daher vor allem der zentrale Nahteil der Linse genutzt, wohingegen durch die Mydriasis beim Blick in die Ferne der periphere Fernteil zur Verfügung steht. ${ }^{13,54}$ Unter dem Prinzip der Diffraktion, was im 18. Jahrhundert von Thomas Young beschrieben wurde, versteht man die Beugung von Lichtstrahlen an einem Hindernis. Diffraktive Linsen besitzen neben einer refraktiven auch eine diffraktive Oberflächenstruktur. ${ }^{64}$ Das Licht wird durch konstruktive Interferenzerscheinungen auf mehrere Brennpunkte verteilt. Die diffraktive Oberfläche besteht aus zirkulären Stufen mit einer Höhe von etwa $2 \mu \mathrm{m}$ auf der Linsenvorder- oder -rückfläche. ${ }^{59}$ Durch Lichtverluste höherer Ordnungen können etwa $20 \%$ des einfallenden Lichts nicht genutzt werden. ${ }^{13,59}$ Bei IOL mit apodisierter diffraktiver Optik nimmt die Stufenhöhe graduell fortlaufend zur Peripherie hin ab, wodurch die Lichtintensität in der Linsenperipherie reduziert und die Abbildungsqualität insgesamt verbessert wird. Der Patient profitiert klinisch von einer Steigerung des Kontrastsehens und einer Reduktion von störenden optischen Phänomenen. ${ }^{19}$ Im Gegensatz zu rein refraktiven Linsen ist das Sehvermögen mit diffraktiven IOL unabhängig vom Pupillendurchmesser und der Zentrierung der Linse. ${ }^{54,64}$ Mit ihnen wird ein besserer Nah- und Intermediärvisus erreicht, wohingegen refraktive Linsen für die Fernsicht besser geeignet sind. ${ }^{49,65}$ Beim Prinzip des „Mix and Match" wird eine ferndominante Linse in das dominante Auge implantiert und eine nahdominante Linse in das kontralaterale Auge. ${ }^{49}$

Falls bei einem Patienten eine Hornhautverkrümmung vorliegt, gibt es verschiedene Möglichkeiten diese operativ zu korrigieren. Ein Astigmatismus bis zu 1,5 dpt kann durch die Hornhautinzision ausgeglichen werden. Da die Ergebnisse dieser Methode nur begrenzt vorhersagbar und beständig sind, sollten bei größeren Werten torische Linsen verwendet werden, die neben der 
sphärischen auch eine torische Komponente besitzen. ${ }^{66}$ Intraoperativ muss unbedingt auf die korrekte Achslage einer solchen Linse geachtet werden. ${ }^{54}$ Eine Abweichung von 30 Grad führt zu einem kompletten Wirkungsverlust der torischen Komponente. ${ }^{29}$ Torische Multifokallinsen liefern ausgezeichnete Ergebnisse hinsichtlich Nah-, Intermediär- und Fernvisus sowie Rotationsstabilität und Vorhersagbarkeit. ${ }^{67}$

Akkommodative Linsen werden aufgrund einer schlechten Vorhersagbarkeit des postoperativen Ergebnisses bisher selten eingesetzt. Ihr Prinzip beruht darauf, dass die physiologische Akkommodationsfähigkeit der Linse imitiert wird, um im Nah-, Intermediär- und Fernbereich scharf zu sehen. ${ }^{13}$ Im Gegensatz zu den Multifokallinsen haben die akkommodativen IOL nur einen Brennpunkt. Die Pseudoakkommodation wird erreicht, indem die Kunstlinse durch die Kontraktion des Ziliarmuskels nach vorne gezogen wird, wodurch die Brechkraft des optischen Systems zunimmt. ${ }^{13,29} \mathrm{Im}$ Vergleich zu monofokalen Linsen führen akkommodative Linsen zu einem besseren Nahvisus und höheren Raten an Brillenfreiheit. ${ }^{68}$ Eine Überlegenheit gegenüber Multifokallinsen konnte bisher nicht gezeigt werden. ${ }^{69}$ Weitere Entwicklungen sind nötig, um diesen Linsentyp zu etablieren. ${ }^{70}$

\subsubsection{Patientenselektion und Aufklärung}

Patienten und Chirurgen können aus einem breiten Spektrum von Kunstlinsen aus verschiedenen Materialien, mit verschiedenen Haptiken und optischen Eigenschaften wählen. Aufgrund der Vielzahl der verfügbaren Modelle ist es wichtig, den Patienten präoperativ ausführlich zu beraten, um gemeinsam mit ihm die optimale Linse auszuwählen. Im Rahmen des Gesprächs müssen die Erwartungen des Patienten eruiert werden. Es sollten konkrete Alltagsaktivitäten, wie Beruf, Freizeitaktivitäten, Computernutzung oder der individuelle Leseabstand, angesprochen werden. ${ }^{13}$ Multifokallinsen sind beispielsweise für Fernfahrer ungeeignet, da diese auch bei Nacht auf einen exzellenten Fernvisus angewiesen sind. 49

Vor allem bei Patienten mit okularen Pathologien sollte vor der Implantation einer Multifokallinse abgewogen werden, ob der Patient geeignet ist und von den 
Vorteilen profitieren kann. ${ }^{49,71}$ Bestimmte Pathologien, wie z. B. ein Keratokonus, eine altersbedingte Makuladegeneration oder eine vorangegangene Amotio retinae, sind Ausschlusskriterien für den Einsatz von Multifokallinsen. ${ }^{49}$

Die Pupille sollte unter mesopischen Bedingungen etwa 3,5-7,0 mm weit sein. Bei zu enger Pupille ist das Sehvermögen mit refraktiven IOL bei schlechten Lichtverhältnissen kompromittiert. Bei diffraktiven Linsen ist eine geringe Pupillenweite mit einer Einschränkung des Fernvisus und des Kontrastsehens assoziiert. Durch eine zu weite Pupille können eine vermehrte Blendempfindlichkeit und Halos nach der Implantation von akkommodativen und multifokalen Linsen auftreten. ${ }^{49}$

Um Enttäuschungen zu vermeiden, muss der Patient darüber informiert werden, welche seiner Wünsche in der konkreten Situation realistisch und umsetzbar sind und welche Erfolgsaussichten bestehen. ${ }^{13}$ Man sollte versuchen, den Patienten nach dem Prinzip „underpromise and overperform“B zu beraten. ${ }^{72}$ Gerade dann, wenn sich der Patient für eine Sonderlinse entscheidet, die von der Krankenkasse nicht übernommen wird, ist eine gründliche Aufklärung mitsamt Dokumentation nötig. Zusammen mit dem Patienten wird entschieden, welche Linse den gewünschten Anforderungen am besten gerecht werden kann. ${ }^{49,54}$ Dem Patienten sollte erklärt werden, dass die optischen Phänomene aufgrund der Neuroadaptation im Verlauf geringer werden, aber dass dieser Prozess einige Zeit in Anspruch nimmt. Er muss außerdem auf die Möglichkeit hingewiesen werden, dass störende Phänomene dauerhaft vorhanden bleiben. ${ }^{73}$

\subsubsection{Präoperative Vermessung der Augen}

Exakte biometrische Messungen sind eine Grundvoraussetzung für den späteren Operationserfolg. Entsprechend des "garbage-in garbage-out"-Prinzips ${ }^{\mathrm{C}}$ können ohne präzise präoperative Ausmessungen der optischen Strukturen keine guten postoperativen Ergebnisse erwartet werden. ${ }^{74}$ Um die Stärke der Linse zu

\footnotetext{
в Übersetzung: weniger versprechen und mehr leisten

c Übersetzung: Unsinn rein - Unsinn raus
} 
berechnen, die implantiert werden soll, werden je nach verwendeter Formel unterschiedliche Eingabedaten benötigt (siehe Abbildung 3).

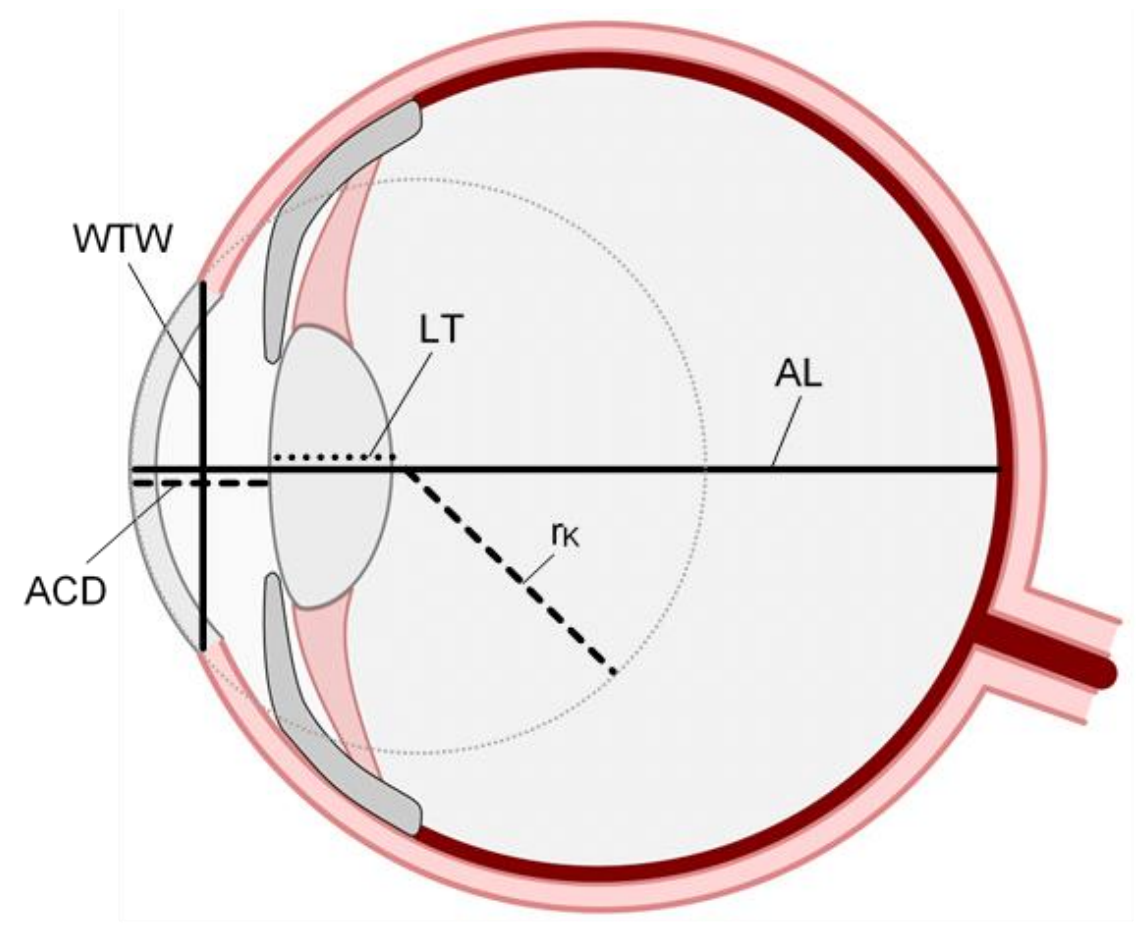

Abbildung 3: Darstellung der Messparameter

(Abbildung verändert nach Grotz ${ }^{75} ; A C D=$ Vorderkammertiefe, $A L=$ Achsenlänge, $L T=$ Linsendicke, $r_{K}=$ Hornhautradius, $W T W=$ Hornhautdurchmesser)

Sowohl der IOLMaster (Carl Zeiss Meditec AG, Jena, Deutschland) als auch der Lenstar LS 900 (Haag-Streit AG, Köniz, Schweiz) bestimmen alle erforderlichen Parameter zur IOL-Kalkulation. Bei der Messung der Achsenlänge $(A L)$ wurde der A-Scan-Ultraschall weitgehend von kontaktfreien, optischen Biometern wie dem IOLMaster oder dem Lenstar abgelöst. ${ }^{76,77}$ Das Prinzip des IOLMasters basiert auf Teilkohärenzinterferometrie mit einer $780 \mathrm{~nm}$ Infrarotlaserdiode, das des Lenstars auf optischer niedrig-kohärenter Reflektometrie (optical low coherence reflectometry, OLCR) mit einer $820 \mathrm{~nm}$ Superlumineszenzdiode. ${ }^{78,79}$ OLCR wird vom Lenstar zusätzlich zur Bestimmung der zentralen Hornhautdicke (central corneal thickness, CCT), der Vorderkammertiefe (anterior chamber depth, ACD), der Linsendicke (lens thickness, LT) und der Dicke der Retina genutzt. ${ }^{78}$ Die ACD wird vom IOLMaster bestimmt, indem ein Lichtspalt in die Vorderkammer geschickt wird und die reflektierten Strahlen auf der 
gegenüberliegenden Seite von einer Kamera registriert werden. ${ }^{80}$ IOLMaster und Lenstar betrachten die Hornhaut als dünne Linse, weshalb nur die Krümmung der Vorderfläche beachtet wird. Die Zwei-Zonen-Keratometrie des Lenstars nutzt 32 Messpunkte, wohingegen die Keratometriewerte (K-Werte) des IOLMasters 500 aus der Analyse von sechs Lichtreflexen in einer optischen 2,3 mm-Zone entstammen. ${ }^{78,79}$ Die Messwerte beider Biometriegeräte unterscheiden sich leicht, wohingegen die Ergebnisse der IOL-Kalkulation vergleichbar sind. ${ }^{81,82}$

Die Pentacam (OCULUS Optikgeräte $\mathrm{GmbH}$, Wetzlar, Deutschland) beruht auf dem Scheimpflug-Prinzip. Neben der Vorderfläche wird auch die Hornhautrückfläche vermessen und die K-Werte basieren auf dem Dicke-Linsen-Modell der Kornea. Die exakte Visualisierung der Hornhaut ermöglicht eine frühzeitige Erkennung und Klassifikation von Pathologien, beispielsweise eines Keratokonus. Durch eventuell veränderte Streueigenschaften werden auch Hornhautund Linsentrübungen detektiert. ${ }^{83}$ Die Pentacam liefert allerdings ausschließlich Daten des vorderen Augenabschnitts und ist daher nicht als alleiniges Messgerät für die IOL-Kalkulation geeignet.

Da die Hornhautbrechkraft nicht direkt gemessen werden kann, wird zuerst der Hornhautradius in zwei Achsen bestimmt. Aus den gemessenen Hornhautradien werden die K-Werte wie folgt von den Keratometern berechnet ${ }^{84}$ :

$$
K=\frac{n_{K}-n_{L}}{r_{K}}
$$

Formel 1: Berechnung der Hornhautbrechkraft ( $K=$ Hornhautbrechkraft, $n_{K}=$ Brechungsindex der Hornhaut, $n_{L}=$ Brechungsindex der Luft, $r_{K}=$ Hornhautradius)

Je nach Biometriegerät werden unterschiedliche Brechungsindizes der Hornhaut verwendet. Die meisten IOL-Formeln, wie die Holladay 1, Sanders-Retzlaff-Kraff/ theoretical (SRK/T), Hoffer Q und Holladay 2, gehen davon aus, dass der Hornhautbrechungsindex, mit dem die Messgeräte die Brechkraft berechnet haben, 1,3375 beträgt. Der Lenstar und die Pentacam nutzen diesen Index von 1,3375 zur Berechnung der K-Werte. Im Gegensatz dazu berechnet der IOLMaster die K-Werte mit einem Index von 1,332. ${ }^{85}$ Bei der Kalkulation der 
Stärke einer Kunstlinse ist daher eine genaue Beachtung der von den Biometern verwendeten Indizes und gegebenenfalls eine Umrechnung essenziell.

Die biometrischen Messungen sollten rechtzeitig vor der Operation durchgeführt werden, um die Möglichkeiten der IOL-Implantation zu besprechen und dem Patienten ausreichend Bedenkzeit zu geben. Damit die Messungen nicht verfälscht werden, sollte der Patient ein bis zwei Wochen vorher auf das Tragen von weichen sowie zwei bis vier Wochen vorher auf das Tragen von formstabilen, harten Kontaktlinsen verzichten. ${ }^{52,72}$

\subsubsection{Formeln zur Kalkulation der Stärke der Intraokularlinse}

Für die Berechnung der Stärke der einzusetzenden IOL können verschiedene Linsenformeln verwendet werden. Einige sind auf den gängigen Biometriegeräten wie dem IOLMaster oder dem Lenstar vorinstalliert.

Alle Formeln benötigen mindestens die Hornhautbrechkraft bzw. den -radius und die AL. Andere Parameter, wie z. B. die ACD, tragen zur Vorhersage der effektiven Linsenposition (ELP) bei. ${ }^{76}$ Die ELP ist die Position der Hauptebene der IOL im Verhältnis zum gesamten Auge und hängt z. B. von der Größe der Kapsulorhexis ab. Bei einer größeren Kapsulorhexis schrumpft die verbliebene Kapsel postoperativ mehr als bei einer kleineren Kapsulorhexis, wodurch die Kunstlinse letztlich weiter vorne im Auge positioniert wird. Die Position der Linsenhauptebene innerhalb der IOL ist abhängig vom optischen Linsendesign. ${ }^{55}$

Die optische ACD ist definiert als der Abstand von der Vorderseite des Hornhautepithels zur vorderen Linsenkapsel (siehe Abbildung 4). ${ }^{55,86}$ Dieser Wert ist abzugrenzen von der anatomischen ACD, dem Abstand zwischen Hornhautendothel und Linsenvorderfläche. ${ }^{78}$ Die Hornhautdicke wird bei optischen Betrachtungen mit einbezogen, da die Hauptebene der Hornhaut im Bereich der Vorderfläche liegt. ${ }^{74}$ Die Hornhauthöhe ist aus optischer Sicht der Abstand vom epithelialen Scheitelpunkt der Hornhaut zur vorderen Irisebene. Die ELP ist der Abstand von der Vorderseite der Hornhaut zur Hauptebene der Kunstlinse, wobei diese als dünne Linse, d. h. ohne nennenswerte Dicke, angesehen wird. Da die $\mathrm{IOL}$ allerdings eine gewisse Dicke hat, ist dieser Wert rein fiktiv und entspricht 
nicht der postoperativen optischen ACD. Die postoperative ACD ist $u$. a. abhängig vom optischen Design der Kunstlinse. ${ }^{55}$

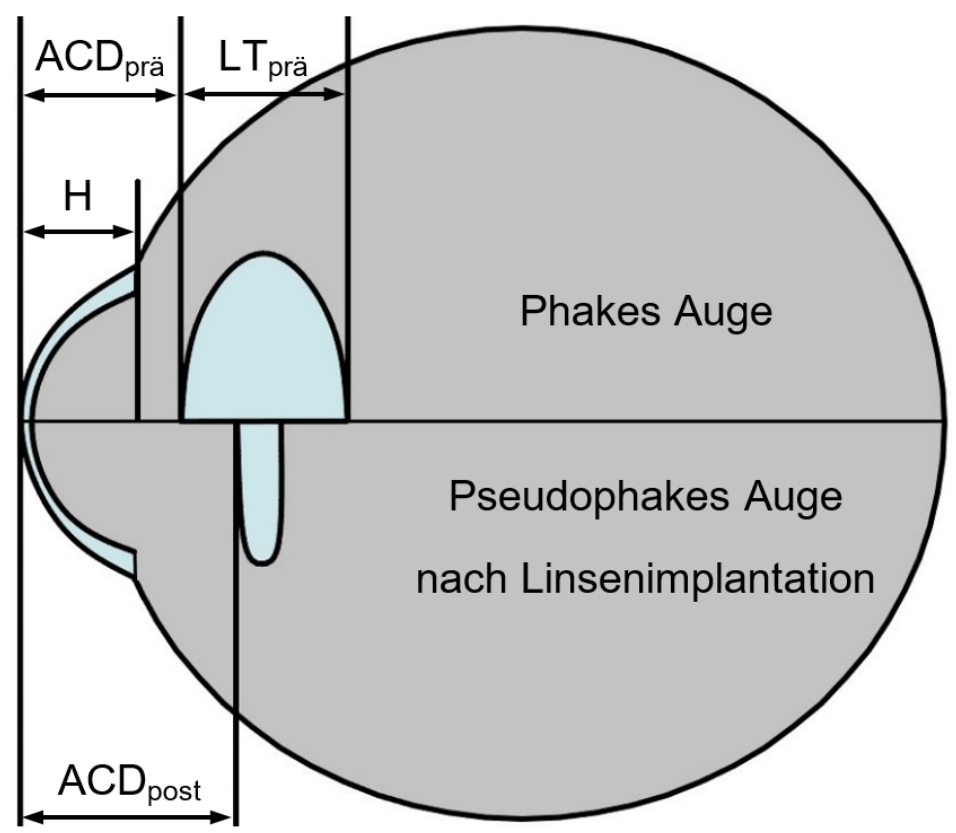

Abbildung 4: Abmessungen des Vorderabschnitts im phaken und pseudophaken Auge (Abbildung verändert nach Olsen ${ }^{55} ; A C D_{\text {prä }}=$ präoperative optische Vorderkammertiefe, $A C D_{\text {post }}=$ postoperative optische Vorderkammertiefe, $H=$ Hornhauthöhe, $L T_{\text {prä }}=$ präoperative Linsendicke)

Der Vollständigkeit halber soll erwähnt werden, dass in älteren Formeln die Hornhauthöhe mit der anatomischen ACD, dem Abstand vom endothelialen Scheitelpunkt der Kornea zur vorderen Irisebene, gleichgesetzt wird. ${ }^{87,88}$ Jede Formel benötigt eine Konstante, die primär abhängig vom optischen Design des implantierten Linsenmodells ist. ${ }^{89}$ Unter dieser Konstanten können aber prinzipiell jegliche systematische Fehler subsumiert werden, die im Prozess der Messung, Berechnung und Operation auftreten, weshalb sie von Olsen treffend als „black box" bezeichnet wurde. Sie ist außerdem abhängig von der Population, in der die Linsenberechnung durchgeführt wird. ${ }^{55}$ Die vom Hersteller angegebene Konstante kann zwar als Ausgangspunkt genutzt werden, führt aber gegebenenfalls zu unzufriedenstellenden Resultaten. ${ }^{50}$ Um optimale Ergebnisse zu erreichen und systematische Fehler $\mathrm{zu}$ minimieren, ist es unbedingt empfehlenswert, die Konstanten der Formeln für jeden Ophthalmochirurgen zu 
optimieren. ${ }^{90,91}$ Durch eine Anpassung der IOL-Konstanten liegt die vorhergesagte Refraktion in $96 \%$ der Fälle um weniger als $1 \mathrm{dpt}$ von der tatsächlichen Refraktion entfernt. Ohne Anpassung ist dies nur in 50\% der Fall. ${ }^{76}$

Zu den theoretischen Formeln der ersten Generation gehören die Colenbrander, Binkhorst, Fyodorov und Hoffer. ${ }^{92-95}$ Sie basieren auf drei Parametern: der AL, der Hornhautbrechkraft und einem konstanten Wert für die ACD. Die SandersRetzlaff-Kraff (SRK) I-Regressionsformel nutzt statt der ACD eine A-Konstante, die vom jeweiligen Linsenmodell abhängt. ${ }^{96}$ Regressionsformeln basieren auf einer empirischen Herangehensweise, nicht auf optischen Modellen. Durch die Analyse bestehender Datensätze werden Regressionskoeffizienten bestimmt, mit denen ein vorhandenes Modell korrigiert wird. Problematisch ist diese Vorgehensweise, wenn der Datensatz, auf dem die Korrektur beruht, nicht repräsentativ und nicht vergleichbar mit den Datensätzen ist, auf die die korrigierte Formel angewendet werden soll. Beispielsweise werden mit dem IOLMaster grundsätzlich längere AL ermittelt als mit der Ultraschallbiometrie, da der IOLMaster den Abstand von der Hornhautvorderfläche zum retinalen Pigmentepithel misst, wohingegen die Ultraschallbiometrie nur den Abstand zur inneren Grenzmembran bestimmt. Eine Regressionsformel, die auf einem Datensatz basiert, bei dem die AL mittels Ultraschall gemessen wurde, kann daher bei der Anwendung auf einen mit dem IOLMaster erhobenen Datensatz nicht zufriedenstellende Ergebnisse liefern. ${ }^{55}$

Die konstante ACD wurde in Formeln der zweiten Generation durch eine von der jeweiligen $A L$ abhängigen $A C D$ ersetzt. ${ }^{55,88}$ Die Binkhorst II sagt die postoperative ACD unter Verwendung einer ACD-Konstanten voraus, die die mittlere ACD bei Implantation eines bestimmten Linsentyps repräsentiert. ${ }^{55}$ Die SRK IIRegressionsformel berechnet eine optimierte A-Konstante unter Beachtung der AL. ${ }^{97}$

Die erste Formel der dritten Generation war die Holladay $1 .{ }^{88}$ Die SRKRegressionsformel wurde überarbeitet und als theoretische SRK/T publiziert. ${ }^{87}$ Die T2 ist eine Weiterentwicklung der SRK/T. ${ }^{98}$ Auch die Hoffer wurde überarbeitet und ist als Hoffer $Q$ verfügbar. ${ }^{48}$

Die Holladay 2 und Haigis sind Formeln der vierten Generation. ${ }^{99,100}$ 
Die Formeln der dritten und vierten Generation haben eine Dünne-Linsen-Formel als Grundlage. ${ }^{101,102}$ Sie sind theoretische, optische Formeln (englisch: vergence formulas) und basieren auf der paraxialen Gaußschen Optik. ${ }^{99,101}$ Sowohl Kornea als auch Linse werden als dünne Linsen betrachtet, die einen Abstand haben, der als optische ACD bezeichnet wird. ${ }^{103}$

Eine Einteilung der neueren Formeln in Generationen wird als nicht mehr angemessen angesehen. ${ }^{99}$

Die Barrett Universal II und die ursprüngliche Olsen werden als Dicke-LinsenFormeln bezeichnet. ${ }^{104-108} \mathrm{Im}$ Gegensatz zu den Dünne-Linsen-Formeln beachten sie die Dicke der Hornhaut und der Linse sowie die unterschiedlichen Krümmungen der Vorder- und Rückflächen. ${ }^{55,102}$ Es werden allerdings nur Strahlen betrachtet, deren optische Achse parallel zur Linsenachse liegt, was man als paraxiales Raytracing bezeichnet. ${ }^{55}$ Die Barrett Universal II wird in der neuen Einteilung zur Gruppe der optischen Formeln gerechnet, wohingegen die neue Variante der Olsen eine eigene Gruppe der exakten Raytracing-Formeln bildet. Sowohl die theoretischen, optischen Formeln als auch die exakten Raytracing-Formeln basieren in geringem Maße auf Regressionsanalysen. ${ }^{99}$

Unter Raytracing versteht man Verfahren zur Strahlenverlaufsberechnung. Im Gegensatz zu Modellen von dünnen und dicken Linsen, d. h. paraxialem Raytracing, können Strahlengänge beim Prinzip des exakten Raytracings auch dann berechnet werden, wenn das einfallende Licht nicht parallel zur Linsenachse auf die Linse auftritt. ${ }^{109}$ Es wird dazu das Snellius-Brechungsgesetz genutzt. ${ }^{74}$ Bezüglich der Vorhersagefähigkeit sind exakte Raytracing-Verfahren besser geeignet, da diese im Gegensatz zu Dicke-Linsen-Formeln zusätzlich Abbildungsfehler wie sphärische Aberrationen der Hornhaut und der Linse beachten. ${ }^{110}$ Sie sollten daher bei der IOL-Kalkulation asphärischer Linsen bevorzugt werden. ${ }^{55}$ Wenn die Brechkraft der Kornea beispielsweise überschätzt wird, wird die Brechkraft der Linse unterschätzt und somit wird die Linsenposition als tiefer angesehen, als es eigentlich der Fall ist. Die vom Hersteller angegebene Linsenstärke entspricht der paraxialen Brechkraft. Es wird davon ausgegangen, dass der Winkel zwischen der optischen Achse und der Linsenachse annähernd null ist. Durch sphärische Aberrationen entspricht diese paraxiale Brechkraft nicht 
unbedingt der wirklichen, effektiven Brechkraft. Beispielsweise ist die effektive Brechkraft einer sphärischen Linse durch Abbildungsfehler höher, wenn die Pupille erweitert ist, und niedriger in Miosis. ${ }^{110}$

Die Olsen-Formel ist in zwei Varianten verfügbar: die OLCR-Version, die nur auf dem Lenstar vorinstalliert ist sowie die auf achsenparallelem und exaktem Raytracing basierte Form, die als Computerprogramm erworben werden kann. ${ }^{111}$ Cooke und Cooke berichten von präziseren Ergebnissen mit dem Computerprogramm als mit der OLCR-Version. ${ }^{112}$ Die OLCR-Version nutzt nur die LT und ACD zur Vorhersage der postoperativen Linsenposition, wohingegen die Raytracing-Form zusätzlich AL und K-Werte verwenden kann. ${ }^{111,113}$

Sehr neue und noch wenig evaluierte Formeln sind die Hill-radiale Basisfunktion (RBF), die Kane und die Emmetropia Verifying Optical-Formula (EVOF). Die Kane basiert auf theoretischer Optik, Regression und künstlicher Intelligenz. Sie soll besonders für Augen geeignet sein, die von den Standardabmessungen abweichen. Zum Zeitpunkt der Studiendurchführung konnte die Kane nur für die Berechnung von sechs Linsenmodellen angewendet werden. ${ }^{114}$ Die EVOF ist eine Dicke-Linsen-Formel basierend auf der Theorie der Emmetropisierung. ${ }^{115}$ Für Berechnungen mit der Kane und der EVOF werden die AL, K-Werte und die ACD benötigt. Bei der Kane muss zusätzlich das Geschlecht angegeben werden. Optional können die LT und die CCT eingesetzt werden. Es wird empfohlen, die A-Konstante zu verwenden, die auch für die SRK/T genutzt wird. ${ }^{114,115}$

Zur Berechnung der Stärke von torischen Linsen sowie bei Augen, an denen bereits ein refraktiver Eingriff vorgenommen wurde, sind spezielle Berechnungsmethoden nötig. ${ }^{76}$ Für torische Linsen sind z. B. der Barrett Toric Calculator oder die Abulafia-Koch-Formel geeignet. ${ }^{116}$ Die Barrett True-K, Masket oder Haigis-L ermöglichen die Berechnung der Linsenstärke nach refraktiven Eingriffen. ${ }^{117}$ Das Problem bei postrefraktiven Augen ist, dass das ursprüngliche Verhältnis zwischen Hornhautvorder- und -rückfläche beeinflusst wurde, wodurch zum einen die K-Werte verändert wurden und zum anderen eine präzise Vorhersage der ELP nicht mehr möglich ist. ${ }^{113}$ Die American Society of Cataract and Refractive Surgery stellt auf ihrer Internetseite Onlinekalkulatoren für 
postrefraktive Berechnungen zur Verfügung. ${ }^{118}$ Ebenso bietet sie einen Rechner für torische Linsen an. ${ }^{119}$

Das Einsetzen einer Kunstlinse mit einer optimalen Stärke ist entscheidend für das postoperative Sehvermögen. Die Auswahl der richtigen Formel spielt daher eine überaus wichtige Rolle. Mehrere Studien haben die Ergebnisse bei Verwendung verschiedener Formeln für Mono- und Bifokallinsen verglichen. ${ }^{12,120,121}$ Es existiert allerdings noch keine Studie, die die verschiedenen Formeln in Zusammenhang mit der quadrifokalen PanOptix evaluiert hat.

\subsection{Zielsetzung der Arbeit}

Die Intraokularlinsenchirurgie bietet viele Möglichkeiten, um den Patienten zu einem optimalen Sehvermögen zu verhelfen. Der erste Schritt ist die Auswahl einer passenden Kunstlinse. Mit der quadrifokalen PanOptix steht eine Linse zur Verfügung, die optimal für Kataraktpatienten geeignet ist, die im Alltag nicht auf eine Sehhilfe angewiesen sein möchten. Ein weiterer wichtiger Schritt ist die präoperative Berechnung der Stärke der einzusetzenden Linse. Eine unpassende Linsenstärke führt zu postoperativen Refraktionsfehlern und gegebenenfalls zur Unzufriedenheit des Patienten. Da die Patientenzufriedenheit die höchste Priorität hat, ist es äußerst relevant die präoperative Berechnung zu optimieren. Wie bereits erläutert stehen hierfür verschiedene Formeln zur Verfügung. In der vorliegenden Studie werden die folgenden neun modernen Formeln evaluiert: Holladay 1, SRK/T, Hoffer Q, T2, Holladay 2, Haigis, Barrett Universal II, Olsen und eine neue Formel, die Hill-RBF. Bis jetzt wurde keine Studie publiziert, die die Präzision der Formeln für die Kalkulation von Tri- oder Quadrifokallinsen untersucht hat. Das Ziel der vorliegenden Studie war es daher, die Genauigkeit dieser Formeln zu bewerten und eine Aussage darüber zu treffen, welche Formel für die Berechnung der quadrifokalen PanOptix am besten geeignet ist. 


\section{Material und Methoden}

\subsection{Patientenkollektiv}

\subsubsection{Studiendesign und Studienpopulation}

Bei der vorliegenden Studie handelt es sich um eine retrospektive konsekutive Fallserie, in deren Rahmen die Daten von Patientenaugen verwendet wurden, die sich einer Femtosekundenlaser-assistierten Operation mit Implantation einer quadrifokalen AcrySof IQ PanOptix TFNT00 unterzogen. Indikationen waren sowohl eine Katarakt als auch der Wunsch nach einem refraktiven Linsenaustausch. Die Patienten wurden an der Klinik für Augenheilkunde der Johann Wolfgang Goethe-Universität Frankfurt am Main operiert. Der Beobachtungszeitraum erstreckte sich über drei Monate nach dem Eingriff.

\subsubsection{Einschluss- und Ausschlusskriterien}

Es wurden retrospektiv alle Datensätze von Patienten ausgewählt, bei denen in der Zeit zwischen November 2015 und Januar 2017 eine Femtosekundenlaserassistierte PanOptix-Implantation durchgeführt wurde. Ausschlusskriterien waren Minderjährigkeit oder eine vorausgegangene Operation der Augen. Patientenaugen mit relevanten okulären Pathologien wurden ebenfalls von der Studie ausgeschlossen. Dazu zählten ein Tumor des Auges, eine zentrale Hornhautnarbe, eine Hornhautendotheldystrophie, eine Cornea guttata oder eine Optikusatrophie. Ein schlechtes bzw. unerwartetes postoperatives visuelles Ergebnis war kein Ausschlussgrund.

\subsubsection{Fallzahlberechnung}

Für die Berechnung der Effektstärke wurden die Werte von Reitblat et al übernommen. ${ }^{82}$ Ein mittlerer absoluter Vorhersagefehler (mean absolute error, MAE) von 0,125 dpt und eine Standardabweichung (standard deviation, SD) von 0,3 dpt wurden als klinisch signifikant angesehen. Um einen relevanten Unterschied zwischen zwei Formeln auf einem Signifikanzniveau von $\alpha=0,05$ und mit einer Teststärke von 0,8 zu detektieren, waren 39 Augen notwendig. 


\subsection{Ablauf der Therapie}

\subsubsection{Präoperative Untersuchungen und Messungen}

Präoperativ wurde eine ausführliche Anamnese durchgeführt. Schwerpunkte lagen hierbei auf dem zeitlichen Verlauf und dem subjektiven Ausmaß der Sehverschlechterung. Es wurde gezielt nach systemischen und okulären Vorerkrankungen, Medikamenteneinnahmen und bereits erfolgten operativen Eingriffen an den Augen gefragt. Abhängig von der Vorgeschichte und dem Patientenwunsch wurde die Anästhesie gewählt.

Die ophthalmologische Untersuchung umfasste eine Inspektion der Augen und ihrer Adnexe, eine Spaltlampenuntersuchung des Vorderabschnitts, eine Funduskopie in Mydriasis sowie eine Messung des Augeninnendrucks mittels Applanationstonometrie nach Goldmann.

Zuerst wurde mit einem Autorefraktometer die objektive Refraktion gemessen. Ausgehend von diesen Werten wurde die subjektive Refraktion durch Refraktionieren mittels eines Phoropters bestimmt. Das sphärische Äquivalent (spherical equivalent, SE) kann gemäß der folgenden Formel aus der gemessenen sphärischen und zylindrischen Refraktion berechnet werden:

$$
\text { sphärisches Äquivalent }=\text { Sphäre }+0,5 * \text { Zylinder }
$$

Formel 2: Berechnung des sphärischen Äquivalents

Die Visusbestimmung umfasste den unkorrigierten und korrigierten Fern- (4 m), Intermediär- $(60 \mathrm{~cm}$ und $80 \mathrm{~cm})$ und Nahvisus $(40 \mathrm{~cm})$ und wurde bei standardisierter Beleuchtung mit 85 Candela/ m² gemäß DIN 58220 durchgeführt. Der Visus wurde mithilfe von Early Treatment Diabetic Retinopathy Study-Tafeln erhoben und in logarithm of the minimum angle of resolution (logMAR) angegeben.

Die Befunde wurden gemeinsam mit dem Patienten besprochen. Anschließend wurde er ausführlich über verschiedene Therapiemöglichkeiten aufgeklärt. Alle in dieser Studie eingeschlossenen Patienten entschieden sich für die Implantation einer quadrifokalen Kunstlinse. 
Die biometrische Vermessung und Keratometrie erfolgte mit dem IOLMaster 500 (Softwareversion 7.1, Carl Zeiss Meditec AG, Jena, Deutschland). Folgende Parameter wurden erfasst: K-Werte, die AL, die ACD und der Hornhautdurchmesser (white to white distance, WTW). Das Scheimpflug-System Pentacam (OCULUS Optikgeräte $\mathrm{GmbH}$, Wetzlar, Deutschland) diente zur Bestimmung der CCT. Zur Kalkulation der Stärke der zu implantierenden IOL wurde die auf dem IOLMaster vorinstallierte Haigis-Formel verwendet. Es wurde die Linsenstärke ausgewählt, bei der die vorhergesagte Refraktion der gewünschten Zielrefraktion am nächsten lag. Bei allen Patienten wurde eine Emmetropie angestrebt.

\subsubsection{Operationsablauf}

Alle Operationen wurden von einem erfahrenen Operateur durchgeführt. Die Eingriffe erfolgten in Tropfanästhesie mit Oxybuprocainhydrochlorid (Conjuncain EDO, 4 mg/ ml Ampullen, Dr. Mann Pharma, Berlin, Deutschland). Für die Hornhautinzision, die Kapsulotomie und die Linsenfragmentation wurde der LenSxFemtosekundenlaser (Alcon Laboratories Inc., Fort Worth, Texas, US) genutzt. Der LenSx ist ein $50 \mathrm{kHz}$-Infrarotlaser mit einer Pulsdauer von 600 bis 800 Femtosekunden, einer zentralen Wellenlänge von $1030 \mathrm{~nm}$ und einer maximalen Pulsenergie von $15 \mu \mathrm{J} .^{122}$ Für den Applanationsvorgang wurde das SoftFit Interface (Alcon Laboratories Inc., Fort Worth, Texas, US) eingesetzt, welches aus einer weichen Kontaktlinse und einer Applanationslinse besteht. Die Kontaktlinse, die zwischen Hornhaut und rigider Applanationslinse aufgebracht wurde, sorgte für eine optimierte Laserstrahlfokussierung. Unter Videokontrolle wurde das Interface an das Auge angesaugt. Der Sog führte gleichzeitig zu einer Fixierung des Auges. Die Planung der Lokalisation und Größe der Schnitte erfolgte mithilfe der Livebilder der integrierten optischen Kohärenztomographie. Zusätzlich zur Clear-Cornea-Hauptinzision wurden zwei Arbeitszugänge für Hilfsinstrumente geschaffen. Der Durchmesser der Kapsulotomie betrug $5 \mathrm{~mm}$. Das Muster der Linsenfragmentierung ist in Abbildung 5 dargestellt. 


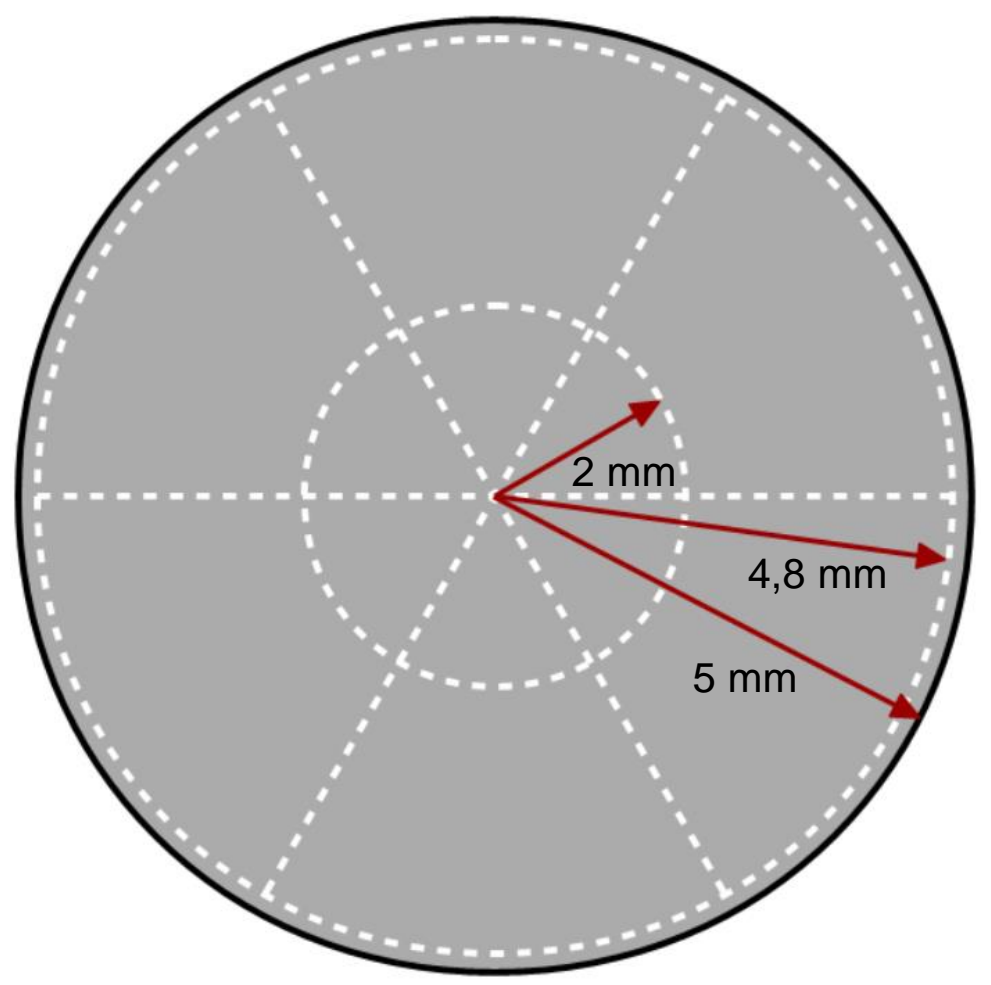

Abbildung 5: Schnittführung der Linsenfragmentierung

In Tabelle 1 sind die für die Operation genutzten Einstellungen des Lasersystems aufgeführt.

Tabelle 1: Einstellungen des Femtosekundenlasers

\begin{tabular}{|l|l|r|}
\hline \multirow{5}{*}{ Korneale Inzision } & Parameter & Wert \\
\hline \multirow{5}{*}{ Kapsulotomie } & Inzisionsweite & $2,2 \mathrm{~mm}$ \\
\cline { 2 - 3 } & Pulsenergie & $7 \mu \mathrm{J}$ \\
\cline { 2 - 3 } & Spot-Separation & $4 \mu \mathrm{m}$ \\
\hline & Schicht-Separation & $4 \mu \mathrm{m}$ \\
\hline & Kapsulotomiedurchmesser & $5 \mathrm{~mm}$ \\
\hline & Pulsenergie & $5 \mu \mathrm{J}$ \\
\hline & Spot-Separation & $4 \mu \mathrm{m}$ \\
\hline & Schicht-Separation & $3 \mu \mathrm{m}$ \\
\hline \multirow{3}{*}{ Linsenfragmentierung } & Anzahl der tangentialen Schnitte & $3,8 \mathrm{~mm}$ \\
\hline & Chop-Durchmesser & 1 \\
\cline { 2 - 3 } & Anzahl der Zylinder & $2 \mathrm{~mm}$ \\
\cline { 2 - 3 } & Zylinderdurchmesser & $5 \mu \mathrm{J}$ \\
\cline { 2 - 3 } & Pulsenergie & $10 \mu \mathrm{m}$ \\
\cline { 2 - 3 } & Spot-Separation & $12 \mu \mathrm{m}$ \\
\cline { 2 - 3 } & Schicht-Separation & \\
\hline
\end{tabular}


Nach Abschluss des Laservorgangs wurde zum Schutz des Hornhautendothels eine viskoelastische Substanz (ProVisc, 1\% Natriumhyaluronat, Alcon Pharma $\mathrm{GmbH}$, Freiburg, Deutschland) in die Vorderkammer injiziert. Es folgte die Hydrodissektion zur Lösung des Linsenkörpers von der Kapsel. Die Ultraschallphakoemulsifikation wurde mit der INFINITY-Phakomaschine (INFINITY Vision System, Alcon Laboratories Inc., Fort Worth, Texas, US) durchgeführt. Nach der Irrigation und Aspiration des Linsenmaterials folgte das erneute Einbringen von viskoelastischer Substanz, um den Kapselsack darzustellen. Mittels eines Injektors (Monarch III Injektor, Alcon Pharma GmbH, Freiburg, Deutschland) wurde die Kunstlinse (AcrySof IQ PanOptix TFNT00, Alcon Laboratories Inc., Fort Worth, Texas, US) durch die Hauptinzision in den Kapselsack eingebracht. Nach dem Absaugen der viskoelastischen Substanz verschlossen sich die Hauptinzision und die Parazentesen selbstständig. Ein Augenverband wurde angelegt.

\subsubsection{Postoperative Untersuchungen und Messungen}

Den Patienten wurden Dexamethason-Neomycinsulfat-Polymyxin-B-sulfatAugentropfen für zwei Wochen und Ketorolac-Augentropfen für sechs Wochen verordnet. Abhängig vom Operationsverlauf fanden in den ersten Tagen mehrfache postoperative Kontrollen statt. Eine eventuell benötigte Sehhilfe wurde frühestens nach drei Wochen verordnet. Nach einem und drei Monaten erfolgte eine Refraktionsbestimmung und eine Visuskontrolle. Zur frühzeitigen Erkennung eines Nachstars wurden jährliche Kontrollen empfohlen.

\subsection{Details der implantierten Intraokularlinse}

Alle Patienten dieser Studie bekamen eine Presbyopie-korrigierende AcrySof IQ PanOptix TFNTO0 implantiert. Der schematische Aufbau ist in Abbildung 6 dargestellt. Die Kunstlinse besteht aus einem hydrophoben Acrylat-MethacrylatCopolymer und filtert ultraviolettes und blaues Licht. Aufgebaut ist die einteilige, faltbare Hinterkammerlinse aus einer zentralen Optik mit einem Durchmesser von $6 \mathrm{~mm}$ und zwei open-loop-Haptiken. Der Gesamtdurchmesser beträgt $13 \mathrm{~mm}$. Die bikonvexe Optik hat eine nicht-apodisierte diffraktiv-refraktive 
Vorderseite und eine sphärische Rückseite. ${ }^{62,123}$ Auf der Vorderfläche befinden sich diffraktive Ringstrukturen innerhalb der zentralen 4,5 mm-Zone, womit vier Fokusse erzeugt werden. Der äußere Ring der Vorderseite ist refraktiv. Die Vorderfläche der Linse führt zu einer negativen sphärischen Aberration von $-0,1 \mu \mathrm{m}$, wodurch die positive Aberration der Hornhaut kompensiert wird. 62,124

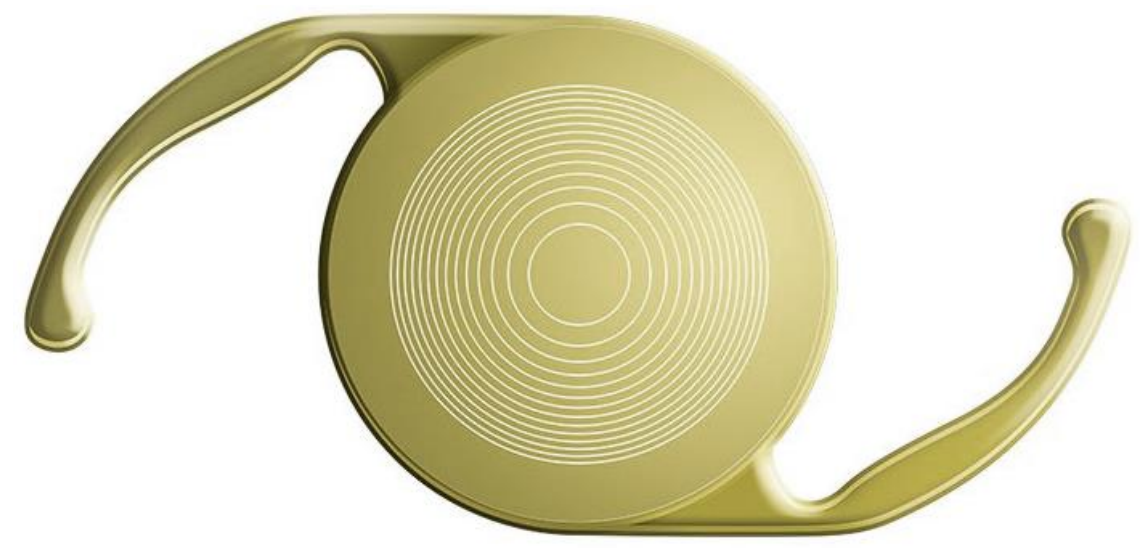

Abbildung 6: PanOptix-Intraokularlinse

(Quelle: Eyefox $U G^{123}$ )

Die Intermediäraddition beträgt $+2,17 \mathrm{dpt}$, die Nahaddition $+3,25 \mathrm{dpt}^{62}$ In einem Bereich von 40 bis $80 \mathrm{~cm}$ zeigt die Defokuskurve ein ausgeprägtes Visusplateau. ${ }^{123,124}$ Der Fokus für den Nahbereich befindet sich bei $42 \mathrm{~cm}$. Der funktionelle Brennpunkt im Intermediärbereich liegt im Gegensatz zu Trifokallinsen bei $60 \mathrm{~cm}$ und nicht bei $80 \mathrm{~cm} .{ }^{124}$ Da dieser Abstand etwa einer Armlänge entspricht, ist die Kunstlinse für die Arbeit im Nah- und Zwischenbereich, z. B. für Computerarbeit oder Kochen, optimal geeignet. Der vierte Brennpunkt bei $120 \mathrm{~cm}$ ist nicht direkt vom Patienten nutzbar. Das Licht der ersten Beugungsordnung fällt mit der nullten, d. h. der Ferne, zusammen. ${ }^{62}$ Es wird also auf den Brennpunkt in der Ferne umverteilt, wie in Abbildung 7 dargestellt. ${ }^{61}$ Letztlich ergibt sich eine trifokale Optik, wodurch die Lichtausbeute und das Sehen im Allgemeinen verbessert werden. ${ }^{61,62}$ Das neue Linsenprinzip wurde von der Firma Alcon ENLIGHTEN genannt, was für "Enhanced Light Energy" steht. Bei einer Pupillenweite von $3 \mathrm{~mm}$ erreichen durch diese Technologie $88 \%$ des Lichts die Retina. ${ }^{62}$ 

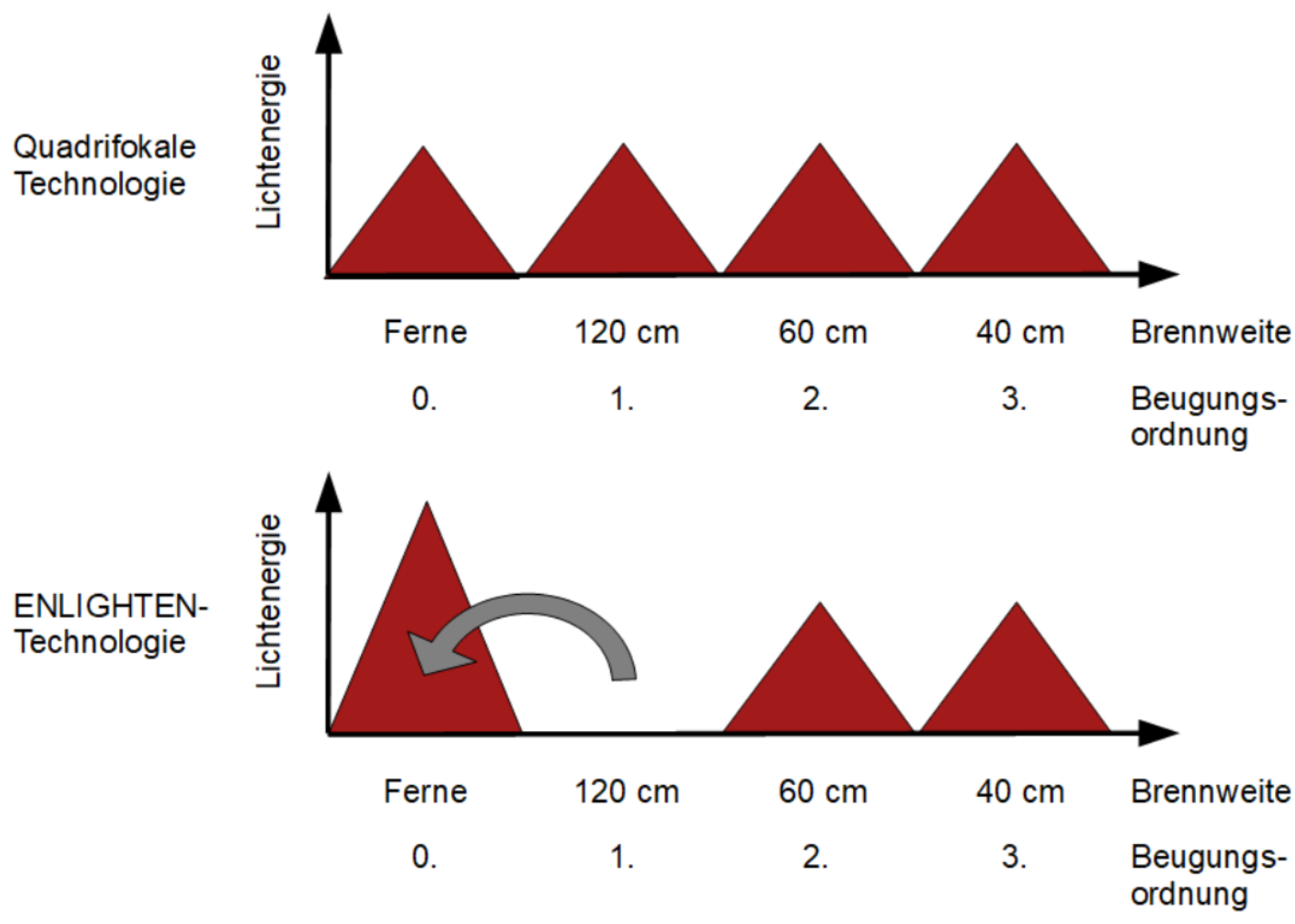

Abbildung 7: Vergleich der klassischen quadrifokalen Technologie mit der ENLIGHTENTechnologie der PanOptix

(Abbildung verändert nach Kohnen ${ }^{125}$ )

Die Verteilung des Lichts auf den Nah-, Intermediär- und Fernbereich in Abhängigkeit von der Pupillenweite ist in Abbildung 8 dargestellt. Ungefähr die Hälfte des Lichts entfällt auf den Fernpunkt, der Rest teilt sich etwa gleichmäßig zwischen Nah- und Intermediärbereich auf.

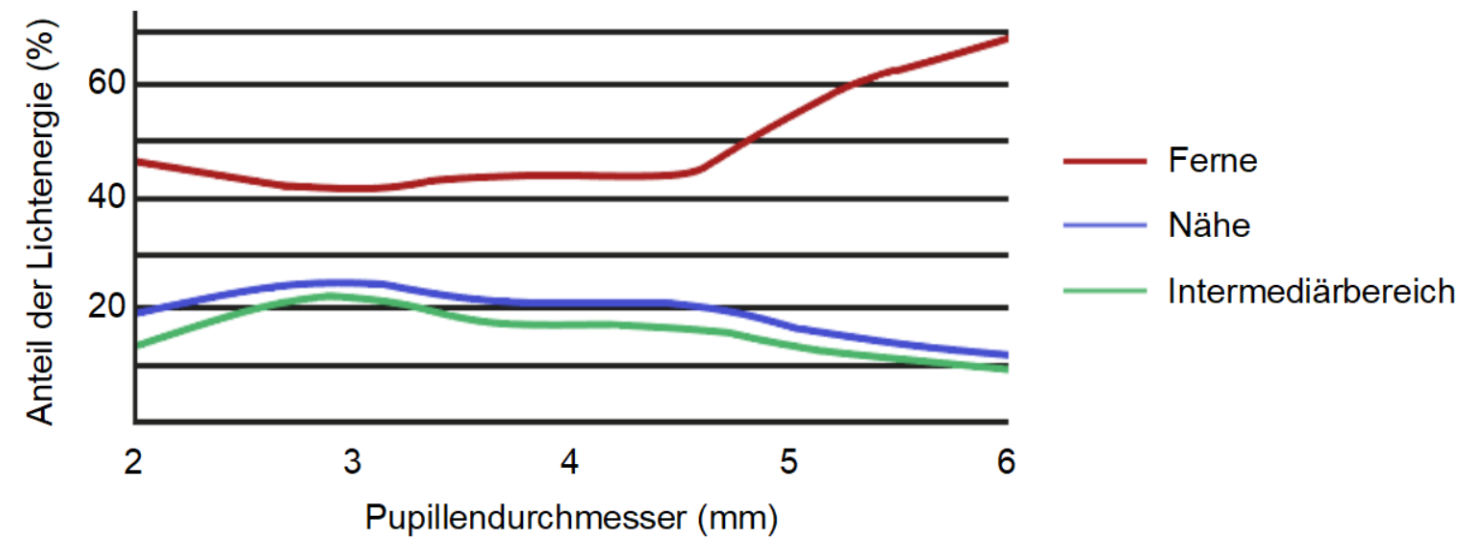

Abbildung 8: Verteilung der Lichtenergie der PanOptix in Abhängigkeit von der Pupillenweite (Abbildung verändert nach Alcon Laboratories Inc.62) 
Durch die nicht-apodisierte Optik ist die Verteilung der Lichtenergie nur geringfügig von der Pupillenweite abhängig. ${ }^{124}$ Als Nachteil bringt dies verstärkte optische Phänomene mit sich, welche die meisten Patienten aber nicht nennenswert einschränken. ${ }^{63,124}$

\subsection{Eigenschaften und Verfügbarkeit der Kalkulationsformeln}

Im Rahmen der vorliegenden Studie wurden die folgenden neun Formel evaluiert: Holladay 1, SRK/T, Hoffer Q, T2, Holladay 2, Haigis, Barrett Universal II, Olsen und Hill-RBF.

Für einen Überblick über die Formeln sei auf Kapitel 4.3.3 ab Seite 35 verwiesen. Die genauen Formeln finden sich soweit verfügbar im Anhang ab Seite 118.

Tabelle 2 zeigt die Parameter, die jeweils eingesetzt werden sollen bzw. können.

Tabelle 2: Einzusetzende Parameter für die Linsenkalkulationsformeln $(A C D=$ Vorderkammertiefe, $A L=$ Achsenlänge, $C C T=$ zentrale Hornhautdicke, $K=$ Hornhaut brechkraft, $L T=$ Linsendicke, $r=$ Hornhautradius, $W T W=$ Hornhautdurchmesser)

\begin{tabular}{|l|l|l|}
\hline Holladay 1 & AL, K & Fakultative Parameter \\
\hline SRK/T & AL, K & \\
\hline Hoffer Q & AL, K & \\
\hline T2 & AL, K & \\
\hline Holladay 2 & AL, K, Alter & ACD, WTW, LT, Refraktion \\
\hline Haigis & AL, r, ACD & \\
\hline Barrett & AL, K, ACD & WTW, LT \\
\hline Olsen & AL, r/ K, ACD & LT, CCT, Pupillendurchmesser \\
\hline Hill-RBF & AL, K, ACD & WTW, LT, CCT \\
\hline
\end{tabular}


Eine Umrechnung der Konstanten der Drittgenerationenformeln, d. h. der Holladay 1 , SRK/T und Hoffer $Q$, ist folgendermaßen möglich ${ }^{88}$ :

$$
\begin{gathered}
\text { Surgeon Factor }=A * 0,5663-65,60 \\
\text { personalisierte Vorderkammertiefe }=A * 0,5836-63,8963
\end{gathered}
$$

Formel 3: Umrechnung der A-Konstanten in Surgeon Factor und personalisierte Vorderkammertiefe $(A=A$-Konstante $)$

Folgende Beziehung gilt zwischen dem Lens Factor der Barrett Universal II und der A-Konstanten des Herstellers ${ }^{105}$ :

$$
\text { Lens Factor }=A * 0,5825-67,6627
$$

Formel 4: Umrechnung der A-Konstanten in Lens Factor ( $A=$ A-Konstante $)$

\subsubsection{Holladay 1}

Die Holladay 1 ist die erste Formel der dritten Generation und berücksichtigt im Vergleich zu vorherigen Generationen die Schätzung der postoperativen Position der Kunstlinse. Die Hornhauthöhe ist definiert als der Abstand des endothelialen Scheitelpunkts der Kornea zur vorderen Irisebene. Sie wird mit der AL korreliert. Zur Berechnung der ELP wird die Summe aus Hornhauthöhe und Abstand der vorderen Irisebene zur Hauptebene der IOL gebildet. Letzterer wird auch als "Surgeon Factor" (SF) bezeichnet und ist die Konstante dieser Formel. Obwohl der SF eine prinzipiell messbare Größe darstellt, sollte er als abstrakte Konstante gesehen werden, da in inn jegliche systematische Fehler einfließen. Unter anderem wird einkalkuliert, dass zur Hornhauthöhe noch die Hornhautdicke hinzugerechnet werden muss. Der SF ist von folgenden Faktoren abhängig: IOLTyp und -Hersteller, Biometriegerät, Keratometer, Platzierung der Linse, Wundverschluss, Operationstechnik und postoperative Gabe von Steroiden. ${ }^{88}$ Die Holladay 1 nutzt den periodischen Wert von $4 / 3$ als Hornhautbrechungsindex. ${ }^{100}$ 


\subsubsection{Sanders-Retzlaff-Kraff/theoretical}

Die SRK/T ähnelt in Großteilen der Holladay 1. Die Vorhersage der postoperativen ACD erfolgt ebenfalls in Abhängigkeit von der AL. Der Abstand der Irisebene zur Linsenebene wird als "Offset" statt als SF bezeichnet. Die A-Konstante ist die Linsenkonstante. Der Einfachheit halber wird die Dezimalzahl 1,333 für den Hornhautbrechungsindex angenommen. Entwickelt wurde die SRK/T auf Basis von theoretischen, optischen Formeln. Mit empirischen Methoden und Regressionsanalysen wurde sie optimiert.87,126 Die initiale Publikation enthielt Fehler, die drei Jahre nach der erstmaligen Veröffentlichung korrigiert wurden. ${ }^{127}$

\subsection{3. $\quad \underline{\text { Hoffer } Q}$}

Die erste Veröffentlichung der Hoffer Q stammt aus dem Jahr 1993.48 Die originale Formel enthält Fehler, die 1994 und 2007 korrigiert wurden. ${ }^{128,129}$ Die Konstante der Hoffer $Q$ ist die personalisierte ACD (personalized anterior chamber depth, pACD). Auch sie sollte als abstrakte Konstante betrachtet werden, obwohl der Begriff "Vorderkammertiefe" einen messbaren Parameter nahelegt. ${ }^{48}$ Als Hornhautbrechungsindex wird der Wert 1,336 genutzt. ${ }^{100}$

Die Holladay 1, SRK/T und Hoffer Q wurden für die vorliegende Analyse in Excel (Version 1802, Microsoft Corporation, Redmond, Washington, US) programmiert. Die Korrektheit der erstellten Excel-Datenblätter wurde überprüft, indem die Berechnungen von zehn Probedatensätzen mit dem IOLMaster 500 wiederholt wurden. Die Ausgabewerte der Excel-Datei stimmten mit denen des IOLMasters überein.

\subsection{4. $\quad \underline{\mathrm{T}}$}

Bei der genaueren Evaluation der SRK/T sind zwei kritische Aspekte aufgefallen. Zum einen ist die Berechnung der korrigierten $A L$ bei einer $A L>36,2 \mathrm{~mm}$ problematisch. An diesem Punkt erreicht die korrigierte AL ihr Maximum von 27,62 mm. Bei einer $A L>36,2 \mathrm{~mm}$ nimmt die korrigierte $A L$ aufgrund einer Quadrierung innerhalb der Formel wieder ab, was aus physiologischer Sicht unlogisch ist 
(vergleiche Formel 13, Seite 120). Zum anderen bleibt die berechnete Hornhauthöhe ab einer bestimmten -brechkraft konstant. Ab einem Hornhautdurchmesser, der dem Doppelten des -radius entspricht, wird der Wert unter der Wurzel gleich null gesetzt (vergleiche Formel 14, Seite 120). Somit soll vermieden werden, dass aus einem negativen Wert die Wurzel gezogen werden muss. Bei steigender Hornhautbrechkraft, d. h. bei abnehmendem Hornhautradius, nimmt die Hornhauthöhe aus diesem Grund nicht mehr weiter zu. Für bestimmte Kombinationen aus AL und K-Werten ist der praktische Einsatz der SRK/T für die IOL-Kalkulation ebenfalls problematisch. Eine Weiterentwicklung war daher angebracht und ist als T2-Formel im Jahr 2010 erschienen. Das erste Problem der AL-Berechnung für lange Augen wurde der Vollständigkeit halber gelöst, indem für alle Augen mit einer $A L>36,2 \mathrm{~mm}$ die korrigierte $A L$ auf $27,62 \mathrm{~mm}$ festgesetzt wurde. Die Berechnung der Hornhauthöhe wurde durch multiple lineare Regression verbessert. Die AL wird dabei direkt eingesetzt. Da keine Berechnung einer korrigierten AL mehr nötig ist, hat sich auch ersteres Problem erübrigt. ${ }^{98}$

Richard Sheard, der Autor der T2, stellt auf seiner Website eine Excel-Datei zur Verfügung, mit der die IOL-Kalkulation durchgeführt werden kann. ${ }^{130}$ Da die Formel allerdings komplett publiziert ist, wurde sie für die vorliegenden Analyse in Excel programmiert, um die A-Konstante einfacher optimieren zu können. Die Korrektheit des erstellten Excel-Datenblatts wurde überprüft, indem die Berechnungen von zehn Probedatensätzen mit der verfügbaren Datei wiederholt wurden. Die Ausgabewerte des Datenblatts stimmten mit denen der veröffentlichten Datei von Sheard überein.

\subsubsection{Holladay 2}

Die Holladay 2 ist bislang nicht publiziert. Aus diesem Grund wurde das Computerprogramm Holladay IOL Consultant Software \& Surgical Outcomes Assessment (HicSoap, Version 2018.0120, Holladay Consulting, Bellaire, Texas, US) genutzt. ${ }^{131}$ Die Standardeinstellungen wurden übernommen. Fakultative Parameter, die in der vorliegenden Studie eingesetzt wurden, sind die ACD, die WTW und die präoperative Refraktion. Das Einsetzen der LT ist bei Augen mit einer $\mathrm{AL} \leq 22 \mathrm{~mm}$ empfohlen. ${ }^{132}$ Aufgrund der fehlenden Messungen wurde die 
LT in der vorliegenden Studie nicht berücksichtigt. Das Programm bietet eine große Auswahl verschiedener Linsen, sodass der Nutzer keine Kenntnisse über die Details der implantierten IOL benötigt.

\subsubsection{Haigis}

Wie bei allen anderen theoretischen Formeln auch, ist die Basis der Haigis die grundlegende IOL-Formel für dünne Linsen. ${ }^{103} \mathrm{Im}$ Vergleich zu den bisher vorgestellten Formeln weist sie allerdings einige Besonderheiten auf. Zum einen wird die postoperative optische $A C D$ auf Basis von $A L$ und präoperativer $A C D$ berechnet. Im Gegensatz zu den anderen Formeln werden dafür keine K-Werte benötigt (vergleiche Formel 26, Seite 124). Zum anderen beinhaltet die Haigis die drei Konstanten a0, a1 und a2. Für die Konstanten a1 und a2 sind folgende Standardwerte empfohlen, die auch in dieser Dissertation verwendet wurden: $\mathrm{a} 1=0,4$ und $\mathrm{a} 2=0,1$. Bei großen Fallzahlen besteht die Möglichkeit, neben a0 auch diese beiden Konstanten per doppelter Regressionsanalyse zu optimieren. ${ }^{100,133}$ Eine weitere Besonderheit ist, dass der Hornhautradius anstatt der Hornhautbrechkraft eingesetzt wird. Dadurch wird vermieden, dass sich Ungenauigkeiten aufgrund der von den Biometriegeräten genutzten Hornhautbrechungsindizes ergeben. Der Brechungsindex der Kornea wurde von Haigis auf 1,3315 festgelegt. ${ }^{133}$

Die Haigis wurde ebenso wie die Formeln der dritten Generation in Excel programmiert.

\subsubsection{Barrett Universal II}

Bei der Barrett Universal II handelt es sich im Gegensatz zu den meisten anderen theoretischen Formeln um eine auf paraxialem Raytracing und Gaußscher Optik basierende Formel für dicke Linsen. ${ }^{113}$ Grundlage ist ein theoretisches Modellauge, bei dem ein Zusammenhang der ACD mit der AL und den K-Werten vorausgesetzt wird. ${ }^{89}$ Die ursprüngliche Version, die Barrett Universal, erforderte die genaue Kenntnis über die Dicke der Kunstlinse, den Brechungsindex und die Lokalisation der primären und sekundären optischen Hauptebene. ${ }^{104}$ In die 
überarbeitete Form müssen die genannten Parameter nicht eingesetzt werden. Der Nutzer benötigt daher keine Angaben bezüglich des Materials und der genauen Konstruktion der IOL. Der Abstand der sekundären Linsenhauptebene von der Irisebene ist abhängig von den Eigenschaften der Linse. Er wird "Lens Factor" genannt und ist die Linsenkonstante der Formel. Die Barrett Universal II wird als "universell“ bezeichnet, da sie laut ihrer Autoren für verschiedene Linsentypen und die gesamte Breite der AL verwendet werden kann. ${ }^{105}$

Die genauen Berechnungsschritte der Barrett Universal II sind nicht publiziert. Daher wurde der IOL-Kalkulator auf der Website der Asia-Pacific Association of Cataract and Refractive Surgeons (Version 1.05) für die Berechnungen genutzt. ${ }^{134}$

\subsubsection{Olsen}

Die Berechnungsschritte der Olsen sind ebenfalls nicht publiziert. Für die Kalkulation wurde das Computerprogramm PhacoOptics IOL power calculation software (Version 1.10.100.2032, IOL Innovations ApS, Aarhus, Dänemark) verwendet. ${ }^{135}$ Das Programm nutzt das Prinzip des achsenparallelen und exakten Raytracings. Das paraxiale Raytracing basiert auf einer Dicke-LinsenFormel und Gaußscher Optik. Das exakte Raytracing bezieht das SnelliusBrechungsgesetz mit ein. Eine große Auswahl an Linsentypen ist in das Programm integriert, sodass die genauen Eigenschaften der implantierten Linse, d. h. Brechungsindex, Dicke und Konfiguration, nicht vom Nutzer eingegeben werden müssen. ${ }^{74}$ Die ACD-Konstante ist die mittlere postoperative ACD für den jeweils gewählten Linsentyp. ${ }^{106}$ Der Wert ist definiert als der Abstand des Scheitels der Hornhaut von der Vorderfläche der Linse. Im Benutzerhandbuch wird hervorgehoben, dass dieser rein anatomische Wert nicht der ELP entspricht. ${ }^{74}$ Ebenso wie bei der Haigis wurden statt der K-Werte direkt die Hornhautradien eingegeben. Die Olsen nutzt einen Hornhautbrechungsindex von $1,376 . .^{74}$ 


\subsubsection{Hill-radiale Basisfunktion}

Der Kalkulator der Hill-RBF ist online verfügbar (Version 2.0). ${ }^{136} \mathrm{Im}$ Gegensatz zu allen anderen Formeln basiert die Hill-RBF auf Mustererkennung und Dateninterpolation statt auf optischen Prinzipien. Sie nutzt damit einen völlig neuen Ansatz im Vergleich zu den herkömmlichen Formeln. Auf Grundlage der $A L$, der ACD und den K-Werten werden Muster erkannt und anschließend Vorhersagen bezüglich der zu implantierenden Linsenstärke getroffen. Um eine größtmögliche Präzision und Sicherheit bei der Berechnung zu gewährleisten, ist der Kalkulator mit einem validierten Grenzmodell kombiniert. Bei bestimmten Kombinationen aus eingetragenen Parametern erscheint eine Warnmeldung, dass eine zuverlässige Vorhersage nur mit einer begrenzten Wahrscheinlichkeit möglich ist. Die Basis der Mustererkennung stellen Datensätze von Patienten dar, deren Messungen mit dem Lenstar erfolgten und die eine monofokale AcrySof IQ SN60WF IOL (Alcon Laboratories Inc., Fort Worth, Texas, US) implantiert bekamen. Es wird angegeben, dass die Formel in dieser Kombination die besten Ergebnisse liefert, aber auch für andere Biometer und IOL angewendet werden kann. ${ }^{137}$

\section{5. $\quad$ Auswertung der Daten}

\subsubsection{Einsetzen der Parameter in die Kalkulationsformeln}

Das Alter der Patienten konnte den Patientenakten entnommen werden. Die übrigen notwendigen Parameter wurden mit dem IOLMaster 500 und der Pentacam erfasst. Der IOLMaster arbeitet mit einem Hornhautbrechungsindex von 1,332. Die Hornhautbrechkraft wird ausgehend vom gemessenen Hornhautradius berechnet und vom Gerät ausgegeben. Bei der IOL-Kalkulation muss darauf geachtet werden, dass einige Kalkulationsformeln von Keratometriemessungen basierend auf einem Index von 1,3375 ausgehen. Bei Berechnungen mit der Barrett Universal II und der Hill-RBF wurden die direkten Ausgabewerte des IOLMasters eingesetzt, da angegeben werden konnte, dass bei der Messung ein Brechungsindex von 1,332 verwendet wurde. Für die Formeln der dritten Generation, die T2 und die Holladay 2 wurden die K-Werte derartig umgerechnet, 
als hätten sie auf einem Index von 1,3375 beruht. In die Haigis und die Olsen wurden die Hornhautradien eingesetzt.

Normalerweise werden die Kalkulationsformeln präoperativ verwendet, um die benötigte Linsenstärke für eine gewünschte Zielrefraktion zu berechnen. In der vorliegenden Studie war jedoch die implantierte Linsenstärke bekannt und es sollte berechnet werden, welche postoperative Refraktion mit dieser Linse erreicht werden würde.

Aus den präoperativ erhobenen Parametern und der implantierten Linsenstärke wurde, unter Verwendung der von der User Group for Laser Interference Biometry (ULIB) empfohlenen Linsenkonstanten (siehe Tabelle 3), für jedes Auge die postoperative Refraktion berechnet.

Tabelle 3: Empfohlene Linsenkonstanten für die PanOptix

(Quellen: Haigis ${ }^{138}$, HicSoap ${ }^{131}$, PhacoOptics ${ }^{135} ; A C D=$ Vorderkammertiefe, $p A C D=$ personalisierte Vorderkammertiefe)

\begin{tabular}{|l|l|r|}
\hline & Linsenkonstante & Empfohlener Wert \\
\hline Holladay 1 & Surgeon Factor & 1,83 \\
\hline Hoffer Q & A-Konstante & 119,1 \\
\hline T2 & pACD & 5,63 \\
\hline Holladay 2 & A-Konstante & 119,1 \\
\hline Haigis & ACD & 5,37 \\
\hline Barrett & a0; a1; a2 & 1,94 \\
\hline Olsen & Lens Factor & 4,65 \\
\hline Hill-RBF & ACD & 119,1 \\
\hline & A-Konstante & 0,$40 ; 0,10$ \\
\hline
\end{tabular}

Der Vorhersagefehler (prediction error, PE) ist definiert als die Differenz zwischen dem tatsächlich erreichten SE und dem vorhergesagten postoperativen SE. Aus den absoluten Werten der PE jedes Auges wurden die MAE der jeweiligen Formeln ermittelt. 


\subsubsection{Optimierung der Linsenkonstanten}

Eine Optimierung der Linsenkonstanten wird empfohlen, um systematische Fehler von der Bewertung der Formel auszuschließen. ${ }^{139}$ Das im Folgenden beschriebene und in dieser Studie genutzte Prozedere ist eine etablierte Vorgehensweise für die Evaluation von IOL-Kalkulationsformeln. 50,120,133 Das Prinzip der Optimierung besteht darin, dass für jede Formel eine entsprechende Linsenkonstante bestimmt wird, deren Verwendung in einem möglichst geringen mittleren Vorhersagefehler (mean error, ME) resultiert. Der genaue Ablauf war jeweils abhängig von der Formel.

Zuerst wurde für jedes Auge der individuelle Wert der Konstanten ermittelt, bei dem der PE null betrug. Da die Holladay 1, SRK/T, Hoffer Q, T2 und Haigis in Excel-Datenblätter programmiert wurden, konnte die Was-wäre-wenn-Analyse mit Zielwertanalyse durchgeführt werden. Die Zielzelle war der PE, der Zielwert war 0,0 und die veränderbare Zelle war die Linsenkonstante. Die Konstanten der übrigen Formeln wurden iterativ, d. h. durch Ausprobieren, bestimmt.

Aus den individuell optimierten Linsenkonstanten der einzelnen Augen wurde der Mittelwert gebildet. Anschließend wurden die Werte bestimmt, die mehr als zwei SD vom Mittelwert abwichen. Der optimierte Wert der Linsenkonstanten einer jeweiligen Formel war der Mittelwert der individuell optimierten Linsenkonstanten der einzelnen Augen mit Ausnahme der mehr als zwei SD vom ursprünglichen Mittelwert abweichenden Konstanten.

Im letzten Schritt wurden alle Berechnungen ein zweites Mal unter Verwendung der optimierten Linsenkonstanten einer jeweiligen Formel durchgeführt.

Zur zuverlässigen Optimierung aller drei Konstanten der Haigis sind mehr als 200 Augen erforderlich. ${ }^{133}$ Aus diesem Grund wurde nur a0 optimiert. Für a1 und a2 wurden die Standardwerte von 0,4 und 0,1 verwendet. 


\subsubsection{Evaluation der Formeln}

Der PE errechnet sich aus dem gemessenen postoperativen SE minus des vorhergesagten SE. Ein positiver PE bedeutet, dass der Patient nach der Operation stärker hyperop ist als vorhergesagt. ${ }^{139}$

Der primär relevante Wert dieser Dissertation ist der MAE. Zusätzlich wurden die SD und das 95\% Konfidenzintervall des MAE, der mediane absolute Vorhersagefehler (median absolute error, MedAE) und der maximale absolute Vorhersagefehler (maximum absolute error, MaxAE) erhoben. Es wurden die Prozentzahlen der Augen bestimmt, deren PE im Bereich von \pm 0,25 dpt, \pm 0,5 dpt, $\pm 1,0 \mathrm{dpt}$ und $\pm 2,0$ dpt lagen. Die primäre Aufgabe der Studie war es, die einzelnen Formeln anhand der genannten Werte miteinander zu vergleichen.

\subsubsection{Statistische Analyse}

Zuerst wurden die absoluten Vorhersagefehler (absolute error, AE) einer jeden Formel mit dem Shapiro-Wilk-Test auf Normalverteilung getestet. Zur Prüfung der Signifikanz der Unterschiede in den $A E$ zwischen den Formeln diente der Friedman-Test. Unterschiede zwischen jeweils zwei Formeln wurden mit dem Wilcoxon-Vorzeichen-Rang-Test für nicht normalverteilte Daten analysiert. Die Bonferroni-Methode wurde zur Korrektur der Alphafehler-Kumulierung für mehrfache paarweise Vergleiche angewendet. Diese statistischen Auswertungen wurden mit der Stata-Software (Version 13, StataCorp LLC, College Station, Texas, US) vorgenommen.

Unterschiede in den Prozentzahlen der Augen, die einen bestimmten Vorhersagefehler aufwiesen, wurden mittels des Cochran Q-Tests auf Signifikanz geprüft. Mehrfache paarweise Vergleiche erfolgten mit der Critical differenceProzedur. Diese Auswertung wurde mithilfe der Excel-Erweiterung XLSTAT (Version 2019.2.3, Addinsoft, Boston, Massachusetts, US) ausgeführt.

Das gewählte Signifikanzniveau betrug $p<0,05$.

Die statistische Auswertung wurde zusätzlich für Subgruppen entsprechend der $\mathrm{AL}$ wiederholt. Die Gruppe der kurzen Augen umfasste Augen mit einer $\mathrm{AL}$ 
$<22,5 \mathrm{~mm}$, die Gruppe der langen Augen solche mit einer AL > 25,5 mm. Die Einteilung erfolgte entsprechend der Studie von Melles et al. ${ }^{140}$ Für die Subgruppenanalyse wurden die Linsenkonstanten nicht erneut optimiert.

\subsection{Ethik und Datenschutz}

Die Zustimmung der Ethik-Kommission des Fachbereichs Medizin der Johann Wolfgang Goethe-Universität Frankfurt am Main lag vor (Antragsnummer 116/16) und die Deklaration von Helsinki wurde beachtet. Die Auswertung der Patientendaten erfolgte anonymisiert. 


\section{Ergebnisse}

\section{1. $\quad$ Charakteristik der Studienpopulation}

75 Augen von 38 Patienten erfüllten die Einschlusskriterien. Das Durchschnittsalter betrug 63,6 \pm 9,2 Jahre (Spannweite: 39 bis 81 Jahre). 21 Patienten waren weiblich (54,7\% der Augen), 17 Patienten männlich (45,3\% der Augen). Acht Patienten (21,3\% der Augen) unterzogen sich einem refraktiven Linsenaustausch. Bei den übrigen Patienten wurde die Operationsindikation aufgrund einer Katarakt gestellt. Die weiteren Charakteristika und präoperativen Werte der Studienpopulation sind Tabelle 4 zu entnehmen. 14 Augen (18,7\%) hatten eine flache Hornhaut $(K<42 \mathrm{dpt})$ und sechs Augen $(8,0 \%)$ eine steile $(K>46 \mathrm{dpt})$. Jeweils neun Augen (12,0\%) waren kurz $(A L<22,5 \mathrm{~mm})$ bzw. lang ( $A L$ $>25,5 \mathrm{~mm})$.

Tabelle 4: Präoperative Werte der Studienpopulation

( $d p t=$ Dioptrien, $m m H g=$ Millimeter Quecksilbersäule, $S D=$ Standardabweichung)

\begin{tabular}{|l|r|r|}
\hline & Mittelwert \pm SD & \multicolumn{1}{|c|}{ Spannweite } \\
\hline Sphäre [dpt] & $0,00 \pm 2,41$ & $-7,00$ bis 6,75 \\
\hline Zylinder [dpt] & $-0,63 \pm 0,54$ & $-2,25$ bis 0,00 \\
\hline Sphärisches Äquivalent [dpt] & $-0,33 \pm 2,43$ & $-7,25$ bis 5,93 \\
\hline Augeninnendruck [mmHg] & $16,16 \pm 3,22$ & 12 bis 24 \\
\hline Hornhautbrechkraft [dpt] & $42,60 \pm 1,79$ & 38,74 bis 46,50 \\
\hline Hornhautdurchmesser [mm] & $12,03 \pm 0,42$ & 11,2 bis 12,9 \\
\hline Zentrale Hornhautdicke $[\mu \mathrm{m}]$ & $555,23 \pm 34,45$ & 441 bis 620 \\
\hline Vorderkammertiefe $[\mathrm{mm}]$ & $3,29 \pm 0,43$ & 2,39 bis 4,29 \\
\hline Achsenlänge $[\mathrm{mm}]$ & $23,80 \pm 1,27$ & 21,57 bis 27,47 \\
\hline
\end{tabular}

Die implantierte Linsenstärke betrug 21,19 \pm 3,32 dpt (Spannweite: 13,5 bis $28,5 \mathrm{dpt})$.

Tabelle 5 zeigt die Ergebnisse der Refraktions- und Visusmessungen drei Monate nach der Operation. 
Tabelle 5: Postoperative Werte der Studienpopulation nach drei Monaten

(CDVA = korrigierter Fernvisus, $C I V A=$ fernkorrigierter Intermediärvisus, $C N V A=$ fernkorrigierter Nahvisus, $d p t=$ Dioptrien, logMAR = logarithm of the minimum angle of resolution, $S D=$ Standardabweichung, UDVA = unkorrigierter Fernvisus, UIVA = unkorrigierter Intermediärvisus, UNVA = unkorrigierter Nahvisus)

\begin{tabular}{|l|r|r|}
\hline & Mittelwert \pm SD & \multicolumn{1}{|c|}{ Spannweite } \\
\hline Sphäre [dpt] & $0,14 \pm 0,37$ & $-0,50$ bis 1,25 \\
\hline Zylinder [dpt] & $-0,30 \pm 0,37$ & $-2,50$ bis 0,00 \\
\hline Sphärisches Äquivalent [dpt] & $-0,02 \pm 0,35$ & $-1,00$ bis 1,13 \\
\hline UDVA [logMAR] & $0,05 \pm 0,13$ & $-0,2$ bis 0,5 \\
\hline UIVA bei 80 cm [logMAR] & $0,15 \pm 0,13$ & 0,0 bis 0,6 \\
\hline UIVA bei $\mathbf{6 0}$ cm [logMAR] & $0,08 \pm 0,12$ & $-0,2$ bis 0,3 \\
\hline UNVA [logMAR] & $0,07 \pm 0,10$ & $-0,2$ bis 0,1 \\
\hline CDVA [logMAR] & $-0,04 \pm 0,08$ & $-0,2$ bis 0,1 \\
\hline CIVA bei 80 cm [logMAR] & $0,14 \pm 0,14$ & $-0,1$ bis 0,6 \\
\hline CIVA bei $\mathbf{6 0}$ cm [logMAR] & $0,06 \pm 0,12$ & $-0,2$ bis 0,3 \\
\hline CNVA [logMAR] & $0,06 \pm 0,09$ & $-0,1$ bis 0,3 \\
\hline
\end{tabular}

Die optimierten Linsenkonstanten können Tabelle 6 entnommen werden. Für die in Excel programmierten Formeln wurden die Werte auf drei Nachkommastellen gerundet. Die Programme bzw. Internetseiten der übrigen Formeln ermöglichten nur eine Rundung auf zwei Nachkommastellen. Im Vergleich zu den empfohlenen Konstanten (vergleiche Tabelle 3, Seite 55) wurden in der vorliegenden Analyse höhere Werte ermittelt. Für die Haigis wurde nur a0 optimiert.

Tabelle 6: Optimierte Linsenkonstanten für die PanOptix $(A C D=$ Vorderkammertiefe, $p A C D=$ personalisierte Vorderkammertiefe $)$

\begin{tabular}{|l|l|r|}
\hline & Linsenkonstante & Optimierter Wert \\
\hline Holladay 1 & Surgeon Factor & 2,042 \\
\hline SRK/T & A-Konstante & 119,408 \\
\hline Hoffer Q & pACD & 5,800 \\
\hline T2 & A-Konstante & 119,345 \\
\hline Holladay 2 & ACD & 5,67 \\
\hline Haigis & a0 (a1; a2) & $1,562(0,4 ; 0,1)$ \\
\hline Barrett & Lens Factor & 2,10 \\
\hline Olsen & ACD & 4,81 \\
\hline Hill-RBF & A-Konstante & 119,33 \\
\hline
\end{tabular}




\subsection{Gesamtanalyse}

\subsubsection{Mittlerer, medianer und maximaler absoluter Vorhersagefehler}

Die folgenden Vorhersagefehler der Berechnungen sind in Tabelle 7 dargestellt: mittlerer PE, mittlerer absoluter PE mit Standardabweichung und 95\% Konfidenzintervall, medianer und maximaler absoluter $\mathrm{PE}$.

Tabelle 7: Vorhersagefehler aller Formeln

$(d p t=$ Dioptrien, $K I=$ Konfidenzintervall, $M A E=$ mittlerer absoluter Vorhersagefehler, MaxAE = maximaler absoluter Vorhersagefehler $M E=$ mittlerer Vorhersagefehler, MedAE $=$ medianer absoluter Vorhersagefehler, $S D=$ Standardabweichung)

\begin{tabular}{|c|c|c|c|c|c|c|}
\hline & $\begin{array}{l}\text { ME } \\
{[\mathrm{dpt}]}\end{array}$ & $\begin{array}{l}\text { MAE } \\
\text { [dpt] }\end{array}$ & $\begin{array}{l}\text { SD } \\
\text { [dpt] }\end{array}$ & $\begin{array}{l}\mathbf{9 5 \%} \mathbf{K I} \\
{[\mathrm{dpt}]}\end{array}$ & $\begin{array}{l}\text { MedAE } \\
\text { [dpt] }\end{array}$ & $\begin{array}{l}\text { MaxAE } \\
\text { [dpt] }\end{array}$ \\
\hline Holladay 1 & 0,03 & 0,38 & 0,39 & 0,$29 ; 0,47$ & 0,27 & 2,21 \\
\hline SRK/T & 0,04 & 0,39 & 0,35 & 0,$31 ; 0,47$ & 0,26 & 1,80 \\
\hline Hoffer $\mathbf{Q}$ & 0,02 & 0,41 & 0,42 & 0,$31 ; 0,51$ & 0,33 & 2,51 \\
\hline T2 & 0,01 & 0,35 & 0,38 & 0,$26 ; 0,44$ & 0,25 & 2,19 \\
\hline Holladay 2 & 0,05 & 0,40 & 0,46 & 0,$29 ; 0,50$ & 0,23 & 2,62 \\
\hline Haigis & 0,01 & 0,38 & 0,51 & 0,$26 ; 0,50$ & 0,21 & 2,96 \\
\hline Barrett & $-0,05$ & 0,29 & 0,31 & 0,$22 ; 0,37$ & 0,20 & 1,30 \\
\hline Olsen & $-0,02$ & 0,34 & 0,45 & 0,$23 ; 0,44$ & 0,19 & 2,62 \\
\hline Hill-RBF & 0,05 & 0,33 & 0,40 & 0,$24 ; 0,42$ & 0,21 & 2,38 \\
\hline
\end{tabular}

Der niedrigste MAE konnte mit der Barrett Universal II erreicht werden, gefolgt von der Hill-RBF. Die Hoffer Q und Holladay 2 führten zu den höchsten MAE. Die geringste SD wies wiederum die Berechnung mit der Barrett Universal II auf. Die höchste SD ergab sich mit der Haigis. Die Olsen, Barrett Universal II und Hill-RBF hatten den geringsten MedAE, die Hoffer $Q$ den höchsten. Der MaxAE war bei Kalkulationen mit der Barrett Universal II mit Abstand am kleinsten. Es folgte die $\mathrm{SRK} / \mathrm{T}$ mit einem um 0,5 dpt höheren MaxAE. Den höchsten MaxAE wies die Haigis auf. 
In Abbildung 9 sind die Verteilungen der absoluten Vorhersagefehler als BoxPlots dargestellt. Das Kreuz repräsentiert den Mittelwert und der horizontale Strich innerhalb der Box den Median. Die Grenzen der Box werden durch den Interquartilsabstand (IQA) festgelegt. Die Antennen reichen maximal bis zum 1,5fachen des IQA. Weiße Punkte stellen milde Ausreißer dar, die zwischen dem 1,5- und 3-fachen des IQA liegen. Schwarze Punkte sind extreme Ausreißer.

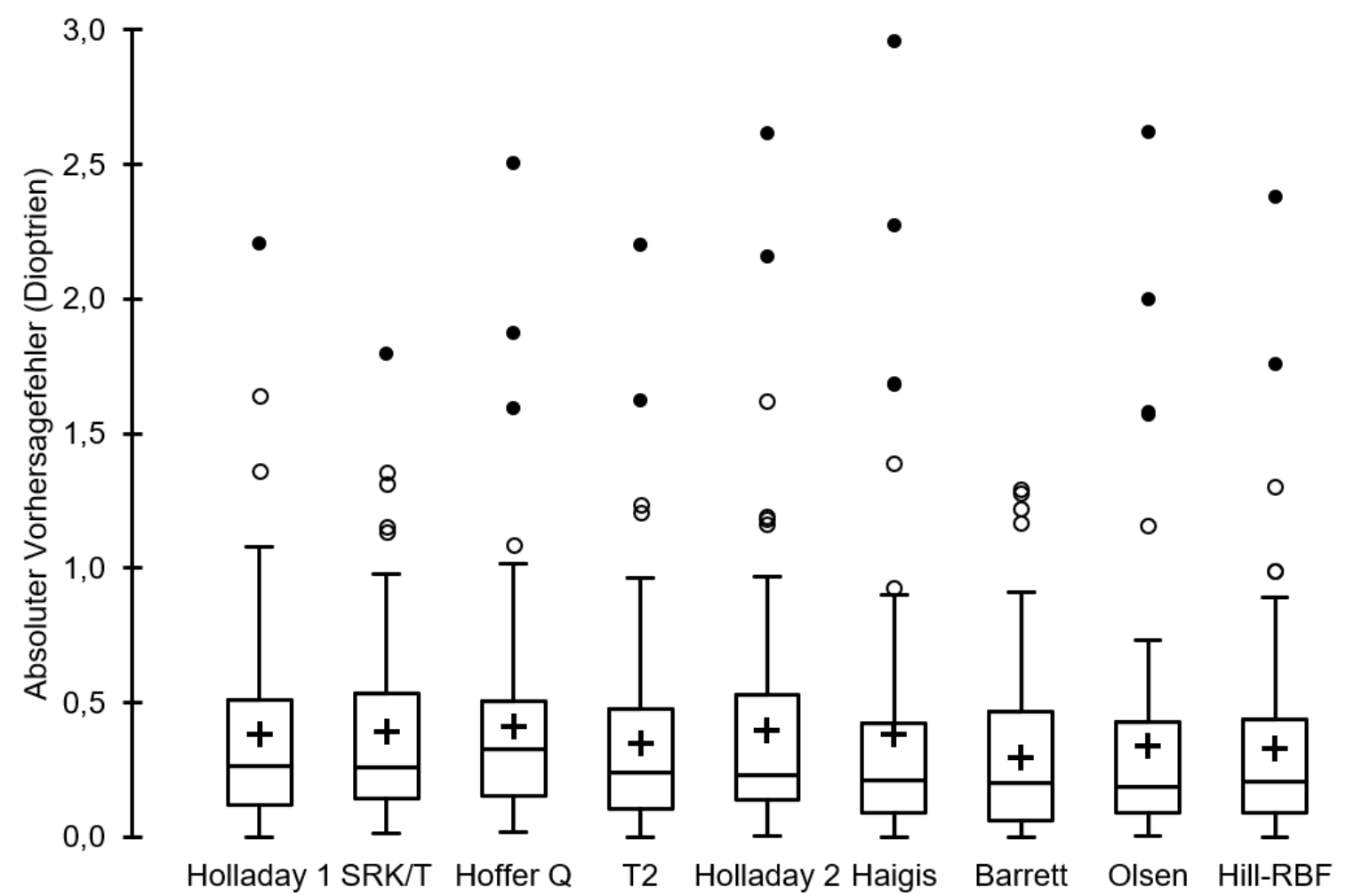

Abbildung 9: Absolute Vorhersagefehler

Insgesamt wurde ein statistisch signifikanter Unterschied zwischen den AE der Formeln gefunden (Friedman-Test: $X^{2}[8]=481,67, p<0,001$ ). Tabelle 8 zeigt die $p$-Werte der Vergleiche von jeweils zwei Formeln mittels des WilcoxonVorzeichen-Rang-Tests. Das Signifikanzniveau lag nach der BonferroniKorrektur bei $p<0,0063$. 
Tabelle 8: P-Werte der Vergleiche von jeweils zwei Formeln

$($ Bar $=$ Barrett Universal II, Hill $=$ Hill-RBF, Hoffer $=$ Hoffer $Q$, Holl $1=$ Holladay 1, Holl $2=$ Holladay 2, * = signifikant nach Bonferroni-Korrektur)

\begin{tabular}{|c|c|c|c|c|c|c|c|c|c|}
\hline & Holl 1 & SRK/T & Hoffer & T2 & Holl 2 & Haigis & Bar & Olsen & Hill \\
\hline Holl 1 & - & 0,447 & 0,031 & 0,178 & 0,812 & 0,401 & 0,026 & 0,092 & $0,006^{*}$ \\
\hline $\mathrm{SRK} / \mathrm{T}$ & 0,447 & - & 0,248 & 0,025 & 0,878 & 0,318 & $0,000^{*}$ & 0,017 & $0,000^{*}$ \\
\hline Hoffer & 0,031 & 0,248 & - & $0,001^{*}$ & 0,358 & 0,070 & $0,002^{*}$ & $0,003^{*}$ & $0,001^{*}$ \\
\hline T2 & 0,178 & 0,025 & $0,001^{*}$ & - & 0,095 & 0,739 & 0,154 & 0,270 & 0,134 \\
\hline Holl 2 & 0,812 & 0,878 & 0,358 & 0,095 & - & 0,620 & $0,003^{*}$ & $0,003^{*}$ & $0,003^{*}$ \\
\hline Haigis & 0,401 & 0,318 & 0,070 & 0,739 & 0,620 & - & 0,108 & $0,004^{*}$ & 0,215 \\
\hline Bar & 0,026 & $0,000^{*}$ & $0,002^{*}$ & 0,154 & $0,003^{*}$ & 0,108 & - & 0,970 & 0,932 \\
\hline Olsen & 0,092 & 0,017 & $0,003^{*}$ & 0,270 & $0,003^{*}$ & $0,004^{*}$ & 0,970 & - & 0,664 \\
\hline Hill & $0,006^{*}$ & $0,000^{*}$ & $0,001^{*}$ & 0,134 & $0,003^{*}$ & 0,215 & 0,932 & 0,664 & 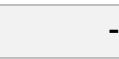 \\
\hline
\end{tabular}

Die Vorhersagegenauigkeit der Hoffer $Q$ war schlechter als die der T2. Die Barrett Universal II schnitt im Vergleich zur SRK/T, Hoffer $Q$ und Holladay 2 besser ab. Die Vorhersagen der Olsen waren exakter als die der Hoffer $Q$, Holladay 2 und Haigis. Die Hill-RBF führte zu statistisch signifikant geringeren AE als die Holladay 1, SRK/T, Hoffer Q und Holladay 2.

\subsubsection{Anteile der Augen innerhalb bestimmter Vorhersagefehler}

In Abbildung 10 sind die Prozentzahlen der Augen visualisiert, deren PE im Bereich von $\pm 0,25 \mathrm{dpt}, \pm 0,5 \mathrm{dpt}, \pm 1,0 \mathrm{dpt}$ und $\pm 2,0 \mathrm{dpt}$ lagen. Die Ergebnisse der Signifikanzprüfungen mittels Cochran $Q$-Tests für $P E$ von $\pm 0,25 \mathrm{dpt}$ und $\pm 0,5 \mathrm{dpt}$ finden sich in den Tabellen 13 und $14 \mathrm{im}$ Anhang auf Seite 126 . 


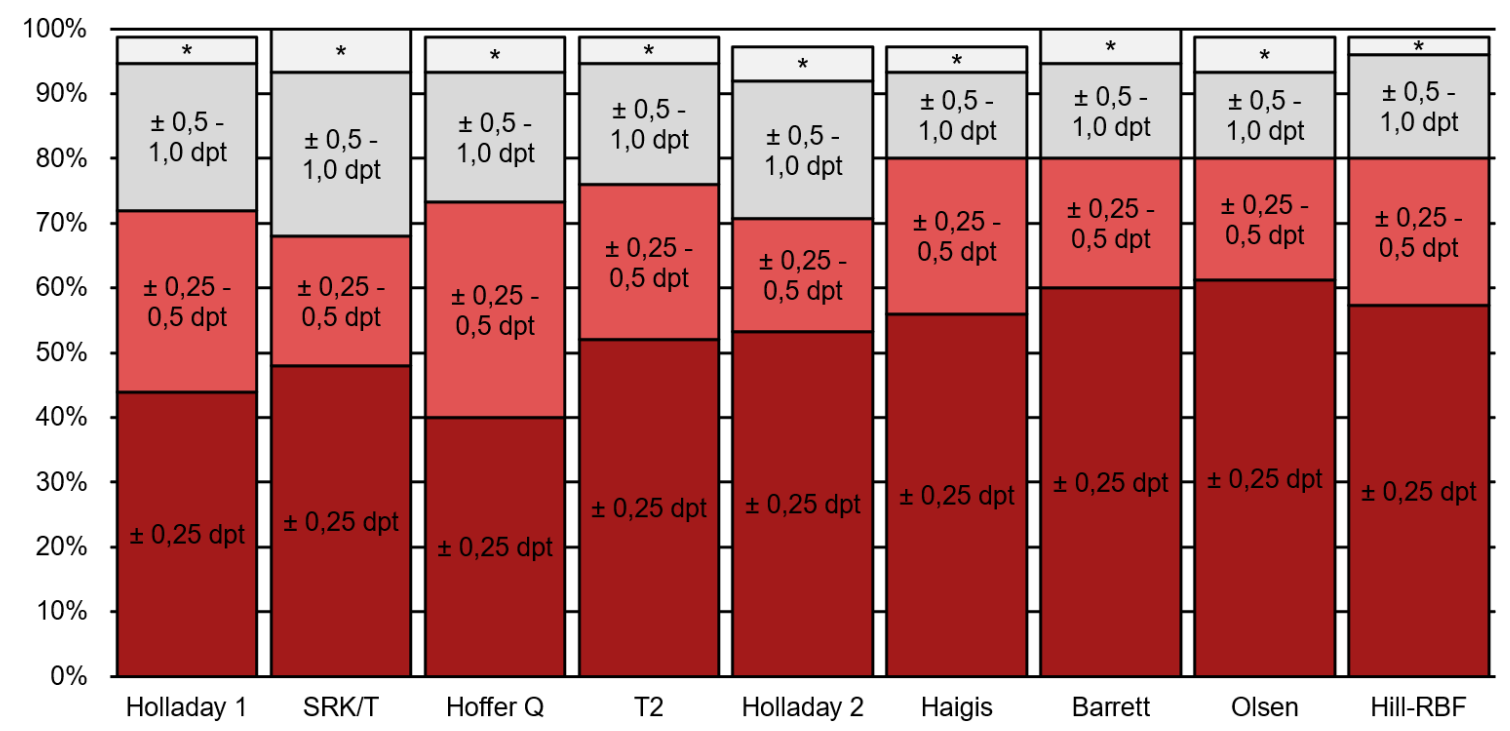

Abbildung 10: Anteile der Augen innerhalb bestimmter Vorhersagefehler $\left(d p t=\right.$ Dioptrien, $\left.{ }^{*}= \pm 1,0-2,0 d p t\right)$

Mit der Olsen und Barrett Universal II hatten mindestens 60\% der Augen einen PE von maximal $\pm 0,25$ dpt. Mit den Formeln der dritten Generation wurden mit unter $50 \%$ niedrigere Prozentsätze erreicht als mit den übrigen Formeln. Die Hoffer Q (40,0\%) war allen Formeln, mit Ausnahme der Holladay 1 (44,0\%) und $\mathrm{SRK} / \mathrm{T}(48,0 \%)$, signifikant unterlegen. Es zeigte sich kein signifikanter Unterschied zwischen den neueren Formeln ab der T2.

Bei Berechnungen mit der Haigis, Barrett Universal II, Olsen und Hill-RBF lagen $80,0 \%$ der Augen innerhalb eines PE von $\pm 0,5$ dpt. Die Verwendung der SRK/T $(68,0 \%)$ und Holladay $2(70,7 \%)$ führte zu einem signifikant geringeren Anteil innerhalb dieses PE im Vergleich zur Haigis, Barrett Universal II, Olsen und HillRBF. Die übrigen Unterschiede waren nicht signifikant.

Für die Anteile der Augen mit einem PE innerhalb von $\pm 1,0 \mathrm{dpt}$ bzw. $\pm 2,0 \mathrm{dpt}$ zeigten sich in den Cochran Q-Tests keine statistisch signifikanten Differenzen zwischen den Formeln. Alle Formeln lagen jeweils bei mindestens $92 \%$ bzw. $97 \%$. 


\subsection{Subgruppenanalyse}

Es wurden zwei Subgruppen entsprechend der AL analysiert. In jeder Subgruppe waren neun Augen, d. h. 24,0\% aller Augen hatten extreme AL. Die Gruppen setzen sich aus Augen mit einer $A L<22,5 \mathrm{~mm}$ bzw. $>25,5 \mathrm{~mm}$ zusammen.

\subsubsection{Mittlerer, medianer und maximaler absoluter Vorhersagefehler}

Die PE der Subgruppenanalyse können den Tabellen 9 und 10 entnommen werden.

Tabelle 9: Vorhersagefehler aller Formeln (Subgruppe mit Achsenlänge $<22,5 \mathrm{~mm}$ ) $(d p t=$ Dioptrien, $K I=$ Konfidenzintervall, $M A E=$ mittlerer absoluter Vorhersagefehler, MaxAE = maximaler absoluter Vorhersagefehler $M E=$ mittlerer Vorhersagefehler, MedAE $=$ medianer absoluter Vorhersagefehler, $S D=$ Standardabweichung)

\begin{tabular}{|c|c|c|c|c|c|c|}
\hline & $\begin{array}{l}\text { ME } \\
{[\mathrm{dpt}]}\end{array}$ & $\begin{array}{l}\text { MAE } \\
\text { [dpt] }\end{array}$ & $\begin{array}{l}\text { SD } \\
\text { [dpt] }\end{array}$ & $\begin{array}{l}\mathbf{9 5 \%} \mathbf{K I} \\
{[\mathrm{dpt}]}\end{array}$ & $\begin{array}{l}\text { MedAE } \\
\text { [dpt] }\end{array}$ & $\begin{array}{l}\text { MaxAE } \\
\text { [dpt] }\end{array}$ \\
\hline Holladay 1 & $-0,36$ & 0,46 & 0,33 & 0,$20 ; 0,71$ & 0,36 & 0,96 \\
\hline SRK/T & $-0,35$ & 0,44 & 0,33 & 0,$19 ; 0,70$ & 0,37 & 0,95 \\
\hline Hoffer Q & $-0,53$ & 0,59 & 0,35 & 0,$32 ; 0,86$ & 0,57 & 1,11 \\
\hline T2 & $-0,38$ & 0,46 & 0,35 & 0,$18 ; 0,73$ & 0,33 & 0,99 \\
\hline Holladay 2 & $-0,51$ & 0,53 & 0,43 & 0,$20 ; 0,86$ & 0,50 & 1,19 \\
\hline Haigis & $-0,67$ & 0,68 & 0,65 & 0,$18 ; 1,19$ & 0,53 & 1,75 \\
\hline Barrett & $-0,34$ & 0,50 & 0,47 & 0,$14 ; 0,86$ & 0,33 & 1,28 \\
\hline Olsen & $-0,53$ & 0,57 & 0,59 & 0,$11 ; 1,02$ & 0,34 & 1,58 \\
\hline Hill-RBF & $-0,24$ & 0,40 & 0,34 & 0,$14 ; 0,66$ & 0,27 & 0,99 \\
\hline
\end{tabular}

In der Subgruppe der kurzen Augen wurde der niedrigste MAE mit der Hill-RBF erreicht, der mit Abstand höchste mit der Haigis. Die SRK/T, Holladay 1, T2 und Hill-RBF hatten einen MaxAE von unter 1,0 dpt. Die höchsten MaxAE lieferten die Haigis und Olsen. 
Tabelle 10: Vorhersagefehler aller Formeln (Subgruppe mit Achsenlänge $>25,5 \mathrm{~mm}$ )

$(d p t=$ Dioptrien, $K I=$ Konfidenzintervall, $M A E=$ mittlerer absoluter Vorhersagefehler, MaxAE = maximaler absoluter Vorhersagefehler $M E=$ mittlerer Vorhersagefehler, MedAE $=$ medianer absoluter Vorhersagefehler, $S D=$ Standardabweichung)

\begin{tabular}{|c|c|c|c|c|c|c|}
\hline & $\begin{array}{l}\text { ME } \\
\text { [dpt] }\end{array}$ & $\begin{array}{l}\text { MAE } \\
\text { [dpt] }\end{array}$ & $\begin{array}{l}\text { SD } \\
\text { [dpt] }\end{array}$ & $\begin{array}{l}95 \% \text { KI } \\
\text { [dpt] }\end{array}$ & $\begin{array}{l}\text { MedAE } \\
\text { [dpt] }\end{array}$ & $\begin{array}{l}\text { MaxAE } \\
\text { [dpt] }\end{array}$ \\
\hline Holladay 1 & 0,38 & 0,44 & 0,25 & 0,$25 ; 0,64$ & 0,39 & 0,87 \\
\hline SRK/T & 0,33 & 0,42 & 0,19 & 0,$28 ; 0,57$ & 0,45 & 0,72 \\
\hline Hoffer Q & 0,21 & 0,24 & 0,25 & 0,$05 ; 0,44$ & 0,12 & 0,76 \\
\hline T2 & 0,07 & 0,21 & 0,16 & 0,$09 ; 0,33$ & 0,18 & 0,52 \\
\hline Holladay 2 & 0,17 & 0,29 & 0,21 & 0,$13 ; 0,45$ & 0,19 & 0,77 \\
\hline Haigis & 0,22 & 0,23 & 0,29 & 0,$01 ; 0,46$ & 0,16 & 0,91 \\
\hline Barrett & 0,09 & 0,20 & 0,18 & 0,$06 ; 0,33$ & 0,12 & 0,62 \\
\hline Olsen & 0,06 & 0,17 & 0,21 & 0,$01 ; 0,34$ & 0,11 & 0,66 \\
\hline Hill-RBF & 0,11 & 0,23 & 0,16 & 0,$12 ; 0,36$ & 0,20 & 0,62 \\
\hline
\end{tabular}

In der Subgruppe der langen Augen wies die Olsen den niedrigsten MAE auf. Die Holladay 1 und SRK/T hatten die höchsten MAE. Bei Verwendung der T2 war der MaxAE deutlich kleiner als bei den anderen Formeln. Der MaxAE war am größten bei Berechnungen mit der Haigis oder Holladay 1.

In den Abbildungen 11 und 12 sind die Verteilungen der AE als Box-Plots dargestellt. Das Kreuz repräsentiert den Mittelwert und der horizontale Strich innerhalb der Box den Median. Die Grenzen der Box werden durch den Interquartilsabstand (IQA) festgelegt. Die Antennen reichen maximal bis zum 1,5fachen des IQA. Weiße Punkte stellen milde Ausreißer dar, die zwischen dem 1,5- und 3-fachen des IQA liegen. Schwarze Punkte sind extreme Ausreißer. 


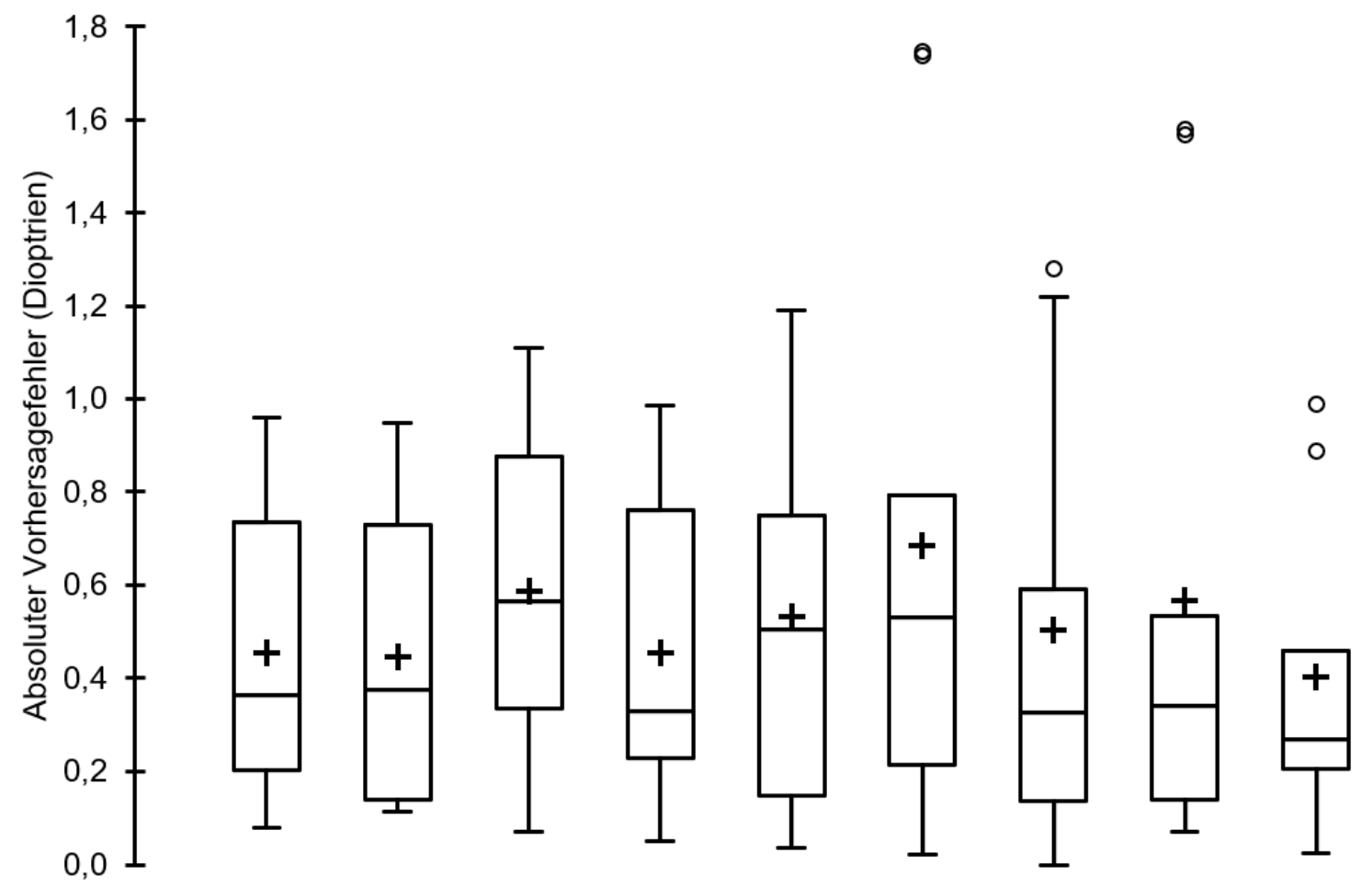

Holladay $1 \mathrm{SRK} / \mathrm{T}$ Hoffer Q T2 Holladay 2 Haigis Barrett Olsen Hill-RBF

Abbildung 11: Absolute Vorhersagefehler (Subgruppe mit Achsenlänge $<22,5 \mathrm{~mm}$ )

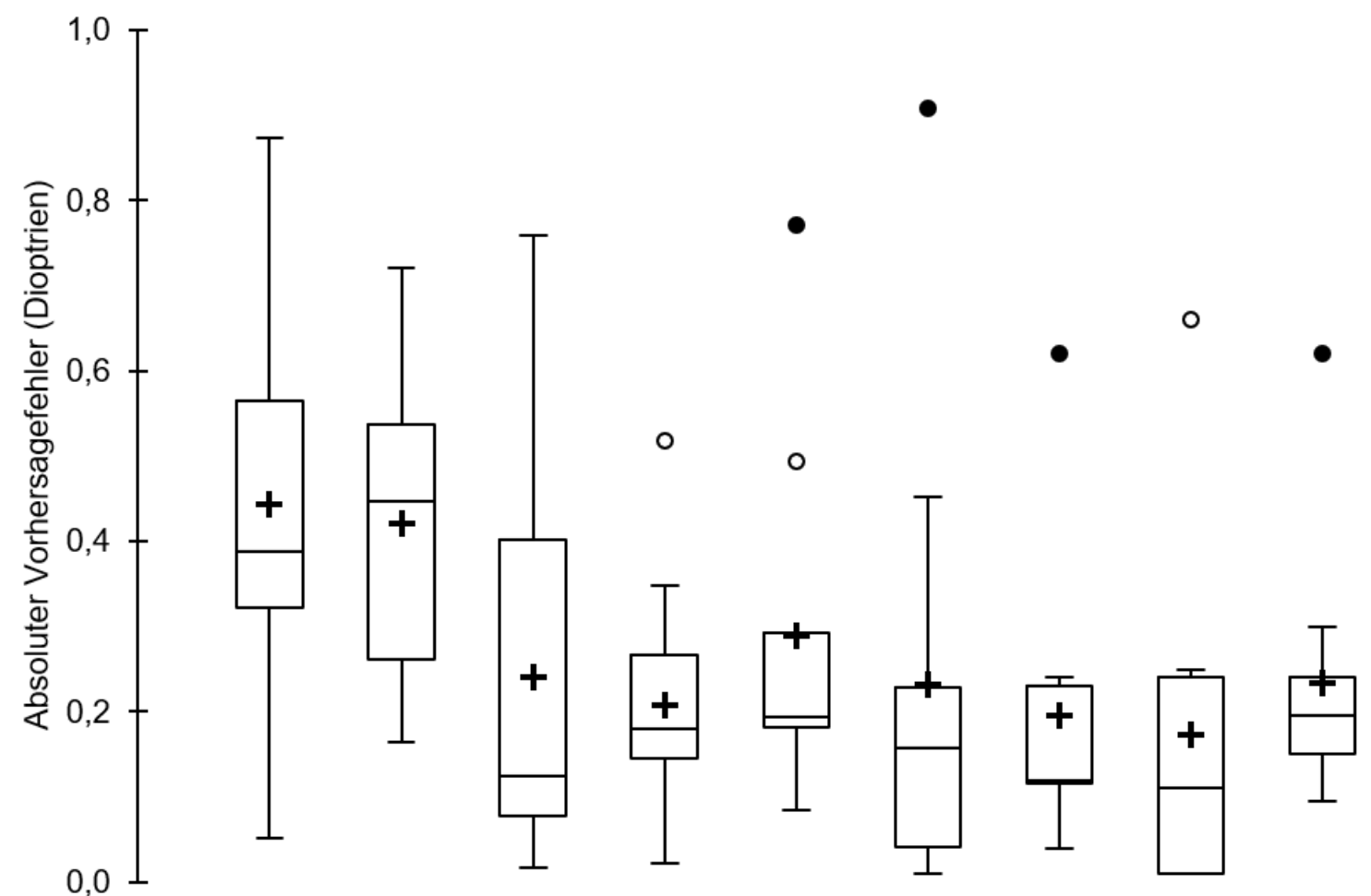

Holladay $1 \mathrm{SRK} / \mathrm{T}$ Hoffer Q T2 Holladay 2 Haigis Barrett Olsen Hill-RBF

Abbildung 12: Absolute Vorhersagefehler (Subgruppe mit Achsenlänge $>25,5 \mathrm{~mm}$ ) 
In den Subgruppen der kurzen (Friedman-Test: $X^{2}[8]=55,14, p<0,001$ ) und langen Augen (Friedman-Test: $X^{2}[8]=31,40, p<0,001$ ) wurden statistisch signifikante Unterschiede zwischen den AE der Formeln gefunden. Der Übersicht halber finden sich die p-Werte der Vergleiche von jeweils zwei Formeln mittels des Wilcoxon-Vorzeichen-Rang-Tests in den Tabellen 15 und 16 im Anhang auf Seite 127. Das Signifikanzniveau lag nach der Bonferroni-Korrektur bei $p<0,0063$. Sowohl für die kurzen als auch für die langen Augen wurden nach der Bonferroni-Korrektur keine Unterschiede bei den Vergleichen von jeweils zwei Formeln gefunden.

\subsubsection{Anteile der Augen innerhalb bestimmter Vorhersagefehler}

In den Abbildungen 13 und 14 sind die Prozentzahlen der Augen dargestellt, deren PE im Bereich von $\pm 0,25 \mathrm{dpt}, \pm 0,5 \mathrm{dpt}, \pm 1,0 \mathrm{dpt}$ und $\pm 2,0 \mathrm{dpt}$ lagen.

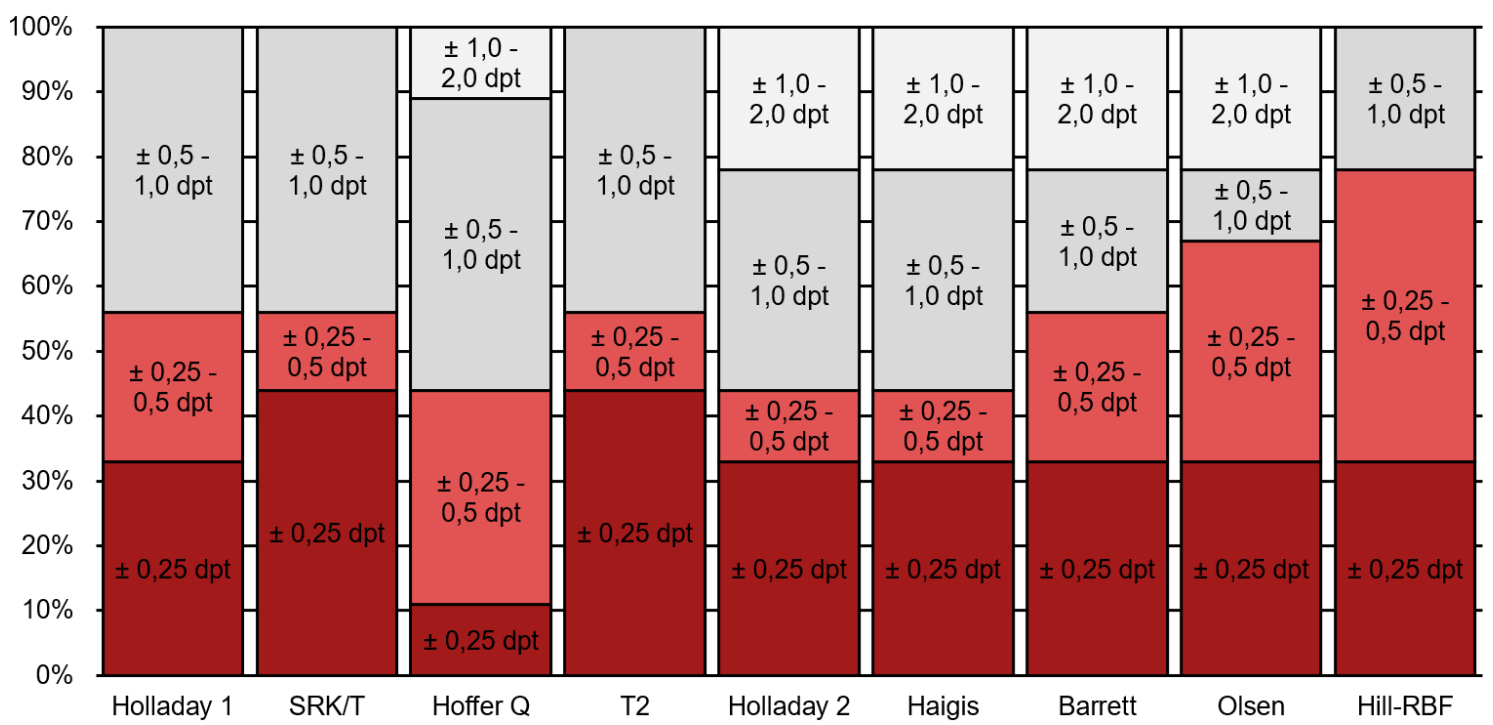

Abbildung 13: Anteile der Augen innerhalb bestimmter Vorhersagefehler (Subgruppe mit Achsenlänge $<22,5 \mathrm{~mm}$ )

$(d p t=$ Dioptrien) 
In der Subgruppe der kurzen Augen wurde die geringste Prozentzahl innerhalb eines PE von $\pm 0,25$ dpt mit der Hoffer $Q(11,1 \%)$, die höchste Prozentzahl mit der SRK/T und T2 (jeweils 44,4\%) erreicht. Bei der Signifikanzprüfung mittels des Cochran Q-Tests stellte sich dieser Unterschied als signifikant heraus. Die HillRBF hatte mit $77,8 \%$ den größten Anteil an Augen innerhalb eines PE von $\pm 0,5 \mathrm{dpt}$, gefolgt von der Olsen mit 66,7\%. Die Unterschiede zwischen den Formeln bezüglich der $P E$ von $\pm 0,5 \mathrm{dpt}$ und $\pm 1,0 \mathrm{dpt}$ waren nicht signifikant. Alle Augen lagen innerhalb eines PE von $\pm 2,0 \mathrm{dpt}$.

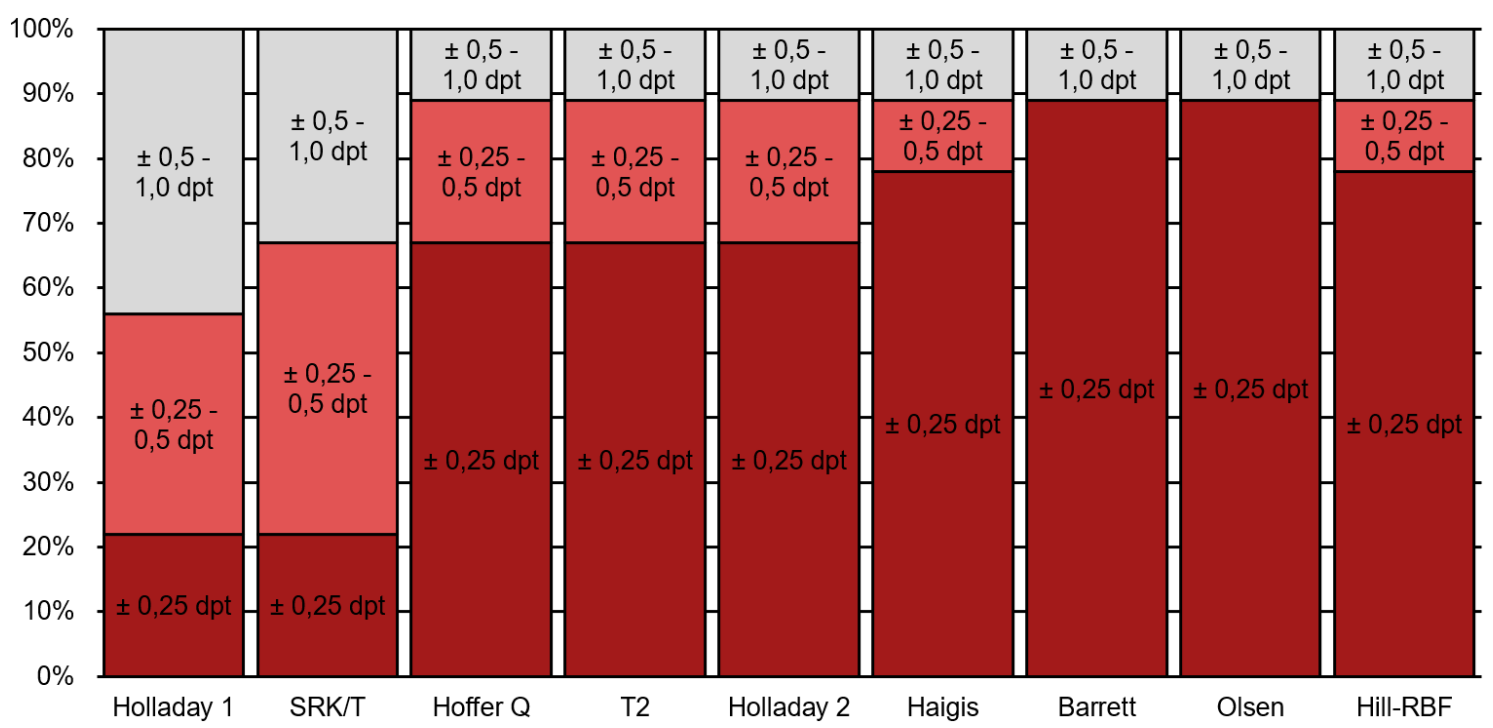

Abbildung 14: Anteile der Augen innerhalb bestimmter Vorhersagefehler (Subgruppe mit Achsenlänge $>25,5 \mathrm{~mm}$ )

(dpt = Dioptrien)

Im Vergleich zu den anderen Formeln lagen in der Subgruppe der langen Augen bei der Verwendung der Holladay 1 und SRK/T mit jeweils 22,2\% deutlich weniger Augen innerhalb eines PE von $\pm 0,25 \mathrm{dpt}$. Dieser Prozentsatz war verglichen mit der Barrett Universal II und Olsen (jeweils 88,9\%) signifikant kleiner. Die Vergleiche zwischen den anderen Formeln zeigten keine statistische Signifikanz im Cochran Q-Test. Mit Ausnahme der Holladay 1 (55,6\%) und SRK/T (66,7\%) hatten 88,9\% der Augen mit allen Formeln einen PE innerhalb von $\pm 0,5 \mathrm{dpt}$. Die Unterschiede waren nicht signifikant. Alle Augen lagen innerhalb eines $P E$ von $\pm 1,0$ dpt. 


\section{Diskussion}

Ich kann lesen, am Computer arbeiten, Auto fahren - alles ohne Brille.

(Ursula Badnjevac, 2015) ${ }^{141}$

Frau Badnjevac wurde der Wunsch vieler Kataraktpatienten erfüllt. Mit der erstmaligen Implantation der quadrifokalen PanOptix durch Professor Dr. Thomas Kohnen im Jahr 2015 wurde ein weiterer Meilenstein in der Kataraktchirurgie erreicht. Weitere Entwicklungen der Operationstechniken, Linsentypen, Messmethoden und IOL-Berechnungen werden den Linsentausch noch sicherer und effektiver machen.

Die vorliegende Studie ist die erste, die verschiedene Formeln zur Berechnung der Linsenstärke der quadrifokalen IOL untersucht hat. Zudem ist bisher keine Arbeit publiziert, die verschiedene Formeln explizit für trifokale Linsen getestet hat.

\subsection{Material und Methoden}

\subsubsection{Vorgaben für Studien zum Vergleich von Kalkulationsformeln}

2015 publizierten Hoffer et al Empfehlungen für Studien, in denen die Genauigkeit verschiedener IOL-Kalkulationsformeln verglichen wird. ${ }^{142}$ In den folgenden Punkten erfüllt die vorliegende Studie diese Anforderungen:

- Eine Beschreibung der Studienpopulation findet sich zu Beginn des Ergebnisteils.

- Alle Patienten bekamen das gleiche Linsenmodell implantiert.

- Die präoperativen Messungen wurden mit einem optischen Biometriegerät vorgenommen. Eine Beschreibung des Keratometriemessverfahrens liegt vor.

- Die ACD wurde definiert. 
- Die postoperative Refraktionsmessung fand nach drei Monaten statt.

- Veraltete Formeln, wie z. B. die SRK I oder II, wurden in die Analyse nicht eingeschlossen.

- Es wird empfohlen, die Formeln nach dem Alphabet zu sortieren, sofern die Reihenfolge keinem anderen Prinzip folgen soll. In der vorliegenden Studie wurden die Formeln nach Aktualität geordnet.

- Eine Überprüfung der Rechenwege der im Rahmen dieser Dissertation programmierten Excel-Datenblätter erfolgte, indem Berechnungen von Testdatensätzen zusätzlich mit dem IOLMaster durchgeführt wurden.

- Die Versionen der Computersoftwares und Websites wurden angegeben.

- Der Vorhersagefehler wurde korrekt als Differenz zwischen dem tatsächlich erreichten postoperativen SE und dem vorhergesagten postoperativen SE berechnet.

- Eine Optimierung der Linsenkonstanten wurde durchgeführt, um den ME zu minimieren.

- Die untersuchten Parameter sind der MAE mit 95\% Konfidenzintervall, der MedAE, der MaxAE und die Anteile der Augen, die einen PE innerhalb von $\pm 0,5 \mathrm{dpt}, \pm 1,0 \mathrm{dpt}$ und $\pm 2,0 \mathrm{dpt}$ erreichten.

In zwei Aspekten weicht die Vorgehensweise dieser Dissertation von den Empfehlungen von Hoffer et al ab. Zum einen wurde nicht nur ein Auge eines Patienten eingeschlossen. Da zum Zeitpunkt der Auswertung wenige Patienten eine Quadrifokallinse implantiert bekommen hatten, wurden beide Augen der Patienten in die Analyse miteinbezogen. Zum anderen basieren die Schlussfolgerungen auf Vergleichen der MAE. Die MedAE werden weniger von Ausreißern beeinflusst als die MAE und sollten daher laut den Autoren zur Bewertung der Formeln herangezogen werden. ${ }^{142}$ In einer Antwort auf das Editorial von Hoffer et al wurden hingegen die MAE als aussagekräftigere Werte postuliert. ${ }^{143} \mathrm{Da}$ in den meisten Studien die MAE zum Vergleich der Formeln herangezogen werden, orientiert sich die vorliegende Studie an letztgenannten Empfehlungen. 
2017 sind neue Vorgaben erschienen. Diese sind im Wesentlichen mit denen von Hoffer et al identisch. Ein Unterschied ist jedoch, dass den MAE mehr Bedeutung zugemessen wird als den MedAE. ${ }^{139}$ Somit stimmt die vorliegende Studie abgesehen von einem Aspekt in allen Punkten mit den Empfehlungen von Wang et al überein. Obwohl die Daten beider Augen eines jeweiligen Patienten verwendet wurden, fand keine Anpassung der statistischen Auswertung statt. Die umstrittene Optimierung der Linsenkonstanten speziell für kurze oder lange Augen hätte in dieser Studie keinen Mehrgewinn gebracht, da zu wenige Augen mit extremen Achsenlängen eingeschlossen waren.

Die Anteile der Augen, die einen PE innerhalb von $\pm 0,25 \mathrm{dpt}, \pm 0,5 \mathrm{dpt}, \pm 1,0 \mathrm{dpt}$ und $\pm 2,0$ dpt erreichten, wurden wie empfohlen in Histogrammen dargestellt. ${ }^{144}$

\subsubsection{Messung der Hornhautbrechkraft}

Die exakte präoperative Vermessung der Hornhaut ist von höchster Relevanz, da die Brechkraft ein entscheidender Faktor bei der Berechnung der Linsenstärke ist.

Die korneale Brechkraft kann nicht direkt gemessen werden. Die Keratometriegeräte können lediglich den Hornhautradius bestimmen. Aus dem Radius wird mittels eines, je nach Keratometriegerät unterschiedlichen, Brechungsindex die Brechkraft berechnet (vergleiche Formel 1, Seite 34). Die meisten Formeln gehen davon aus, dass die Keratometer mit einem Hornhautbrechungsindex von 1,3375 arbeiten. Der Lenstar nutzt diesen Index von 1,3375 zur Berechnung der K-Werte, obwohl Olsen schon 1986 zeigte, dass der wahre Brechungsindex eher bei 1,3315 liegt. ${ }^{145}$ Der IOLMaster rechnet hingegen mit einem Index von 1,332. ${ }^{85}$ In der vorliegenden Studie wurde besonders darauf geachtet, den richtigen Hornhautindex einzustellen oder die gemessenen Werte wenn nötig umzurechnen (vergleiche 5.5.1, Seite 54).

Bei der Keratometrie wird nicht direkt der zentrale Bereich ausgemessen, sondern eine Zone mit einem Durchmesser von etwa 2-3 mm. Bei einer unregelmäßigen Hornhautoberfläche ist es daher möglich, dass die gemessenen K-Werte nicht der zentralen Brechkraft entsprechen. ${ }^{55}$ Besonders bei Patienten, 
bei denen bereits ein Eingriff an der Kornea durchgeführt wurde, stellt die korrekte Linsenberechnung daher oft ein Problem dar. Der Grund dafür ist zum einen die unregelmäßige Hornhautoberfläche nach der Operation. Bei einer keratorefraktiven Korrektur einer Myopie wird durch Stromaabtrag beispielsweise der zentrale Teil der Kornea abgeflacht. Wird nun der periphere Teil vermessen, so wird die Hornhaut als steiler betrachtet, als sie es tatsächlich zentral ist. Dadurch, dass von einer stärkeren kornealen Brechkraft ausgegangen wird, wird die Linsenstärke zu gering gewählt und der Patient ist nach der Operation hyperop. ${ }^{55,72}$ Zum anderen ist relevant, dass bei der Messung des IOLMasters nur die Beschaffenheit der Hornhautvorderfläche beachtet wird. Ein refraktiver Eingriff ändert das als konstant vorausgesetzte Verhältnis der Krümmung von Vorder- und Rückfläche der Kornea, sodass bei der Vernachlässigung der Rückseite falsche Werte ausgegeben werden. ${ }^{146}$ Patienten, bei denen bereits eine Augenoperation durchgeführt worden war, wurden jedoch in der vorliegenden Studie ausgeschlossen, um ungenaue Berechnungen und falsche Schlussfolgerungen aufgrund von präoperativen Messfehlern zu vermeiden.

Durch die bei der Operation vorgenommene Hornhautinzision wird ein geringfügiger Astigmatismus induziert. ${ }^{147}$ Das Ausmaß dieses Astigmatismus kann bei der präoperativen Messung und Berechnung der Linsenstärke nicht beachtet werden und daher zu unerwarteten Visusergebnissen führen. ${ }^{145}$

\subsubsection{Vorhersage der postoperativen Linsenposition}

Heutzutage ist die ungenaue Vorhersage der postoperativen Linsenposition der Hauptgrund für postoperative Refraktionsfehler. Die Optimierung dieser Voraussage ist daher seit längerer Zeit Gegenstand der Forschung. ${ }^{77}$

Die Formeln der dritten Generation, d. h. die Hoffer Q, SRK/T und Holladay 1, und die T2 berechnen die phake ACD und somit die Implantatposition basierend auf der AL und der Keratometrie. Die Annahme, dass ein kurzes Auge grundsätzlich eine geringe $A C D$ und eine flache Hornhaut aufweist, konnte jedoch widerlegt werden. ${ }^{113}$ Ein ähnliches Problem tritt auf, wenn die AL des Auges z. B. durch Buckelchirurgie „verkürzt“ wurde und die anatomischen Verhältnisse somit verändert wurden. ${ }^{55}$ Vor allem bei kurzen Augen kann es zu einer unpräzisen 
Berechnung der Linsenstärke kommen, wenn die präoperative ACD nicht berücksichtigt wird. ${ }^{113,148} \mathrm{Da}$ kein proportionaler Zusammenhang zwischen präoperativer AL und ACD besteht, war zu erwarten, dass die Formeln, die auf mindestens drei Parametern basieren, besser abschneiden. Für die Formeln der dritten Generation hat sich diese Annahme bestätigt. Die T2 lieferte entgegen der Erwartungen gute Ergebnisse, ohne dass die ACD eingesetzt wurde. Aus den genannten Gründen empfiehlt sich nichtsdestotrotz die Verwendung von Formeln, bei denen die ACD berücksichtigt wird. Die schlechten Ergebnisse der Holladay 2 widersprachen der prinzipiellen Annahme, dass solche Formeln, die mehr Parameter zur Berechnung verwenden, die ELP besser vorhersagen können und somit exaktere Ergebnisse liefern.

Die prä- und postoperativen Werte der ACD stimmen nur selten überein. Im Alter verschiebt die zunehmend dickere kristalline Linse die vordere Linsenkapsel nach vorne, wodurch die Vorderkammer flacher wird. ${ }^{74}$ Es ist daher schwierig, die postoperative ACD auf Basis der präoperativ gemessenen ACD exakt vorherzusagen. ${ }^{88}$ Eine kürzlich von Ning et al publizierte Möglichkeit wurde bisher nicht im Rahmen der IOL-Kalkulation getestet. Die Arbeitsgruppe entwickelte zwei Regressionsformeln zur Vorhersage der postoperativen ACD. Eine dieser Formeln bezieht neben der präoperativen ACD zusätzlich die LT mit ein. ${ }^{149}$ Goto et al publizierten eine Regressionsformel, bei der die postoperative ACD aus der $A L$, der präoperativen $A C D$ und dem Abstand der Hornhautrückfläche zur Verbindungslinie zwischen den Kammerwinkeln berechnet wurde. ${ }^{150}$ Shammas und Shammas entwickelten eine neue IOL-Kalkulationsformel, welche statt der ACD und der LT die Dicke des Linsenkerns und den Abstand der Hornhautvorderfläche zur Vorderseite des Linsenkerns nutzte. ${ }^{151}$ Die Vorschläge von Goto et al sowie Shammas und Shammas haben sich im klinischen Alltag ebenfalls noch nicht etabliert. Ein Fehler bei der Ausmessung der ACD und somit eine falsche Berechnung der IOL-Stärke wirkt sich bei kürzeren Augen stärker auf den postoperativen Refraktionsfehler aus als bei langen Augen. ${ }^{55}$

Das Einsetzen der ACD zusammen mit der LT ermöglicht eine bessere Vorhersage der Implantatposition. ${ }^{106,113}$ Statt der typischen ACD-Konstanten kann die IOL-Kalkulation mit dem PhacoOptics-Computerprogramm mit einer C-Konstanten durchgeführt werden. In diesem Fall erfolgt die Berechnung der 
Position des Linsenzentrums entsprechend des folgenden Zusammenhangs ${ }^{106,110}$ :

$$
I O L_{Z}=A C D_{p r a ̈}+C * L T
$$

Formel 5: Berechnung der Position des Linsenzentrums $\left(A C D_{\text {prä }}=\right.$ präoperative Vorderkammertiefe, $C=C$-Konstante eines bestimmten Linsentyps, $I O L z=$ Position des Linsenzentrums, $L T=$ Linsendicke)

Die C-Konstante eines bestimmten Linsentyps kann rückwirkend bestimmt werden, indem Testlinsen implantiert und die anderen drei Variablen gemessen werden. Die ermittelte C-Konstante kann anschließend für die präoperative Vorhersage der Position des IOL-Zentrums genutzt werden. Eine C-Konstante von 0,5 bedeutet, dass sich die Mitte der Kunstlinse an der gleichen Stelle befindet wie das Zentrum der kristallinen Linse vor der Operation. Das C-Konstante-Prinzip wurde in der vorliegenden Studie nicht angewandt, da die LT nicht gemessen wurde. Der große Vorteil des Prinzips besteht darin, dass die Berechnung der postoperativen Linsenposition unabhängig von den K-Werten ist, was vor allem bei Augen nach einer refraktiven Hornhautchirurgie relevant ist. ${ }^{74}$ Diese Augen wurden jedoch in der vorliegenden Studie ohnehin ausgeschlossen.

Die Wichtigkeit der LT bei der Berechnung der Linsenstärke ist umstritten. Laut Olsen spielt sie für die Vorhersage der postoperativen ACD eine äußerst wichtige Rolle. ${ }^{106}$ Gale et al sehen sie auf dem vorletzten Platz in der Liste der Parameter, die einen Einfluss auf die Genauigkeit der Berechnungen haben. ${ }^{152}$ Iribarren et al fanden einen signifikanten Zusammenhang zwischen dem postoperativen Refraktionsfehler und der LT. Dieser war jedoch geringer als der Zusammenhang zwischen dem Refraktionsfehler und der AL bzw. der K-Werte. ${ }^{153}$ In dieser Dissertation konnte die LT aufgrund der Verwendung des IOLMasters 500 nicht eingesetzt werden. Die Messung ist nur mit dem IOLMaster 700 möglich. In einer Studie von Lam wurde gezeigt, dass bei Berechnungen mit der Holladay 2 in 30\% der Fälle eine andere Linsenstärke implantiert worden wäre, hätte man die LT tatsächlich gemessen, anstatt eine Annährung über die lineare Formel von Hoffer zu verwenden. ${ }^{154}$ Die Studien von Srivannaboon et al und Reitblat et al fanden keinen Unterschied in der Präzision der Holladay 2 und Barrett Universal II mit 
und ohne das Berücksichtigen der LT. ${ }^{82,155} \mathrm{Im}$ HicSoap-Programm selbst wird das Einsetzen der $L T$ nur bei Augen mit einer $A L \leq 22 \mathrm{~mm}$ empfohlen. Für längere Augen ergibt sich kein Vorteil. ${ }^{132}$ Obwohl die LT bei den Berechnungen mit der Olsen und Barrett Universal II nicht verwendet wurde, lieferten diese Formeln in der vorliegenden Analyse gute Ergebnisse. Die Berücksichtigung der LT scheint unter theoretischen Gesichtspunkten relevant, wohingegen die Auswirkungen auf die klinischen Ergebnisse kritisch betrachtet werden können.

Ein postoperativer Refraktionsfehler beruht vermutlich auf der falschen Vorhersage der Linsenposition. Aktuell gibt es verschiedene Ansätze, um das Problem der ELP-Berechnung zu lösen. Da beide Augen einer Person im Regelfall symmetrisch sind, erscheint es zum einen sinnvoll, den Vorhersagefehler des ersten Auges dazu zu nutzen, um die einzusetzende Linsenstärke für das Partnerauge zu korrigieren. ${ }^{156,157}$ Zum anderen kann die postoperative ACD des ersten Auges bei der IOL-Kalkulation des zweiten Auges eingesetzt werden. ${ }^{158}$ Außerdem ist es möglich, für das erste Auge postoperativ eine optimierte Linsenkonstante zu ermitteln und diese für die Kalkulation der Linsenstärke des Partnerauges zu nutzen. ${ }^{157}$ Eine Formel zur Berechnung der postoperativen ELP wurde 2018 ebenfalls vorgeschlagen. ${ }^{159}$ Trotz der vielversprechenden Grundideen existiert bisher keine etablierte und unkomplizierte Möglichkeit, diese Erkenntnisse zur Verbesserung der IOL-Kalkulation umzusetzen. Es ist jedoch zu erwarten, dass diese Ansätze zukünftig in der Praxis Verwendung finden werden.

\subsubsection{Umgang mit den optimierten Linsenkonstanten}

Nur Augen mit einem postoperativen korrigierten Fernvisus von 6/12 nach Snellen bzw. 0,03 logMAR oder besser sollten in die Optimierung der Linsenkonstanten einbezogen werden. ${ }^{50}$ Obwohl alle Augen dieser Studie einen deutlich besseren Fernvisus hatten, sollten die ermittelten optimierten Linsenkonstanten nicht im klinischen Alltag für die Linsenberechnung der PanOptix genutzt werden. Erst ab einem Datensatz von etwa 100 Augen werden die optimierten Linsenkonstanten als zuverlässig betrachtet und sind somit für die praktische Verwendung geeignet. ${ }^{50}$ Dieser Wert variiert zudem je nach Formel. 
Für die Hoffer $Q$ sind laut Aristodemou et al 86 Augen ausreichend, für die SRK/T werden mindestens 243 Augen benötigt. ${ }^{90}$ In der vorliegenden Studie diente die Optimierung ausschließlich dazu, die systematischen Fehler zu eliminieren und somit eine verlässliche Vergleichbarkeit der Formeln zu ermöglichen. Alle im Rahmen dieser Analyse ermittelten Linsenkonstanten waren höher als die, die von der ULIB empfohlen werden. Die Verwendung der niedrigeren vorgeschlagenen Konstanten hätte zu einem niedrigeren vorhergesagten SE und somit zu einem positiveren PE geführt. Ein Grund für die Abweichung von den vorgegebenen Linsenkonstanten könnte die geringe Fallzahl sein. Unter Umständen war das Patientenkollektiv dieser Dissertation nicht vergleichbar mit der Population, auf der die Angaben der ULIB basieren.

\subsection{Ergebnisse}

\subsection{1. $\quad$ Aussagekraft der evaluierten Endpunkte}

Zu Beginn der Diskussion der Ergebnisse wird die Aussagekraft der erhobenen Endpunkte kurz im Einzelnen erläutert, um die Bedeutung der Ergebnisse dieser Analyse besser einordnen zu können.

Der ME gibt an, in welchem Ausmaß die Augen durchschnittlich myoper oder hyperoper waren als vorausgesagt. ${ }^{112} \mathrm{Er}$ ist ein Maß für das Vorliegen systematischer Fehler. ${ }^{139} \mathrm{Um}$ diese systematischen Fehler aus der Interpretation der Ergebnisse auszuschließen, sollten bei der Evaluation von IOL-Kalkulationsformeln die Linsenkonstanten optimiert werden. ${ }^{139,142}$ Dadurch werden ME von annähernd null erreicht. Obwohl eine Optimierung in der vorliegenden Studie durchgeführt wurde, betrugen die ME nicht exakt 0,0 dpt. Der Grund liegt darin, dass bei der Optimierung die Augen ausgeschlossen wurden, deren individuell optimierte Konstanten mehr als zwei SD vom Mittelwert abwichen. Diese Augen wurden aber in der abschließenden Analyse wieder einberechnet.

Der MAE ist ein einfach zu ermittelnder und häufig genutzter Parameter, um IOLKalkulationsformeln zu vergleichen. ${ }^{120} \mathrm{Er}$ wird nur gering von Ausreißern beeinflusst. ${ }^{143}$ Die SD des MAE ist ein Maß für die Präzision der Vorhersage. ${ }^{50,139}$ Sie ist unabhängig von der Optimierung der Linsenkonstanten, wird jedoch stärker 
von Ausreißern beeinflusst. ${ }^{12,143}$ Der Grund dafür ist, dass bei der Berechnung dieses Wertes das Quadrat der Differenz des AE jedes Auges vom MAE miteinbezogen wird. ${ }^{143}$ Die SD ist relevant, da Ausreißer möglichst vermieden werden sollten. ${ }^{139}$

Neben den MAE gibt es die Möglichkeit, Formeln anhand der MedAE zu vergleichen. Bei kleinen Fallzahlen folgt die Verteilung der AE keiner Gaußschen Normalverteilung. ${ }^{139}$ In diesem Fall stellt der MedAE den zentralen AE besser dar als der MAE. Der MedAE berücksichtigt keine Ausreißer, die jedoch für die Bewertung der Formel relevant sind. ${ }^{142}$

Ob die MAE oder die MedAE für die Beurteilung der Ergebnisse der Linsenkalkulationsformeln besser geeignet sind, ist umstritten. Da die meisten Studien die MAE analysieren, wurde sich im Rahmen dieser Dissertation dafür entschieden, die Interpretation primär auf den Vergleich der MAE zu stützen.

Die Anteile der Augen, die innerhalb eines PE von $\pm 0,5$ dpt lagen, sind die relevantesten. Bei einem postoperativen SE von weniger als $\pm 0,5 \mathrm{dpt}$ kann man von Brillenfreiheit ausgehen. ${ }^{160}$ Diese Prozentzahlen können zusammen mit dem MaxAE eine Einschätzung geben, welche Einschränkungen die Patienten haben und ob gegebenenfalls eine zweite Operation zum Linsenaustausch erforderlich ist. ${ }^{112}$

\subsubsection{Relevanz der Gesamtanalyse}

Insgesamt konnten mit allen Formeln gute Ergebnisse erreicht werden. Da allerdings gerade bei Multifokallinsen eine Perfektionierung der Ergebnisse anzustreben ist, sollten auch kleine Unterschiede bei der Wahl einer Formel zur IOL-Kalkulation beachtet werden.

Mit Ausnahme von drei Parametern schnitt die Barrett Universal II in allen untersuchten Endpunkten am besten ab. Die Olsen war ihr in zwei Aspekten überlegen: Sie hatte einen geringeren MedAE und einen größeren Anteil an Augen innerhalb eines PE von $\pm 0,25$ dpt. Mit der Hill-RBF erreichten mehr Augen einen PE innerhalb von $\pm 0,5 \mathrm{dpt}$. Die genannten Differenzen zur Barrett Universal II waren jedoch klinisch nicht relevant. Der MaxAE der Barrett 
Universal II war um mehr als 0,5 dpt geringer als der der anderen Formeln. Zudem wurden wenig Ausreißer verzeichnet (vergleiche Abbildung 9, Seite 62).

Die Haigis lieferte bei der Betrachtung des MedAE gute Ergebnisse. Der MAE sowie die SD waren jedoch verhältnismäßig hoch, sodass man davon ausgehen kann, dass mit dieser Formel einige Ausreißer aufgetreten sind.

Von den vier Formeln mit den niedrigsten MAE hatten die Olsen und Hill-RBF höhere SD als die T2 und Barrett Universal II, was sich dadurch erklären lässt, dass auch mit diesen beiden Formeln mehr Ausreißer vorhanden waren.

Der primäre Endpunkt dieser Dissertation waren die Unterschiede im MAE zwischen den Formeln. Die Barrett Universal II hatte den geringsten MAE. Die Differenzen zu den Ergebnissen der SRK/T, Hoffer $Q$ und Holladay 2 waren signifikant. Die Hill-RBF führte zu signifikant geringeren AE als die Holladay 1 , SRK/T, Hoffer $Q$ und Holladay 2. Die Olsen lieferte bessere Vorhersagen als die Hoffer Q, Holladay 2 und Haigis. Die klinische Relevanz der Unterschiede zwischen den Formeln ist fraglich. Die Spannweite des MAE reicht von 0,29 dpt bis zu 0,41 dpt. Der Unterschied zwischen der besten und schlechtesten Formel beträgt folglich 0,12 dpt. Zum Vergleich sollte man beachten, dass Linsenstärken nur in 0,5 dpt-Schritten angeboten werden. Eine geringfügige Verbesserung der Vorhersage führt folglich, aufgrund der begrenzten Umsetzbarkeit der Erkenntnisse, nicht zwingend zu besseren Ergebnissen, da trotz exakterer Berechnung der nötigen Linsenstärke gegebenenfalls die gleiche Linse eingesetzt werden muss. Zudem kann das postoperative SE nur in 0,125 dpt-Schritten angegeben werden. Die gemessene Refraktion ist daher relativ ungenau im Vergleich zu den Unterschieden zwischen den einzelnen Formeln. Systematische Verzerrungen wurden jedoch durch die Optimierung der Linsenkonstanten ausgeglichen.

Frühestens nach einem Monat kann man erwarten, dass die Refraktion einen stabilen Zustand erreicht hat. ${ }^{50}$ Aus diesem Grund wurde im Rahmen der Dissertation höchstwahrscheinlich die endgültige Refraktion für die IOL-Berechnung verwendet. Aufgrund sinnvoller Patientenselektion ergaben sich wenige refraktive Überraschungen. In der vorliegenden Studie wiesen lediglich 9,3\% der Augen ein postoperatives SE von mehr als $\pm 0,5$ dpt auf. In 90,7\% kann man somit davon ausgehen, dass Brillenfreiheit erzielt wurde. ${ }^{160}$ 
Die vorgegebenen Grenzwerte von Gale et al wurden mit allen Formeln erreicht. Demnach sollten $55 \%$ bzw. $85 \%$ der Augen innerhalb eines PE von $\pm 0,5 \mathrm{dpt}$ bzw. $\pm 1,0$ dpt liegen. ${ }^{152}$

\subsubsection{Relevanz der Subgruppenanalyse}

Die Linsenberechnung bei Augen mit extremen AL stellt häufig ein Problem dar. Um einen Anhaltspunkt zu erhalten, welche Formeln für diese Patienten gut geeignet sind, wurde eine Subgruppenanalyse für kurze und lange Augen durchgeführt.

Der ME repräsentiert systematische Fehler und soll durch Optimierung der Linsenkonstanten möglichst reduziert werden. Aus Gründen der Praktikabilität wurde für die Subgruppenanalyse jedoch keine Optimierung durchgeführt. Im klinischen Alltag ist es wahrscheinlicher, dass für alle Augen, unabhängig von der $\mathrm{AL}$, dieselbe Konstante verwendet wird. Die ME der kurzen Augen waren bei allen Formeln negativ. Insgesamt waren die Patienten folglich stärker myop als vorausgesagt. Für die langen Augen zeigte sich das Gegenteil. Die ME aller Formeln waren positiv, d. h. die Patienten waren stärker hyperop als vorhergesagt. Obwohl grundsätzlich eine Emmetropie anzustreben ist, ist ein myopes Ergebnis eher akzeptabel als ein hyperopes.

In der Subgruppenanalyse der kurzen Augen ist auffällig, dass die Holladay 1 und SRK/T sowie die T2 und Hill-RBF die besten Ergebnisse erzielten. Die beiden erstgenannten Formeln der dritten Generation lieferten in der Gesamtanalyse und der Analyse der langen Augen wesentlich schlechtere Vorhersagen. Der Anteil der Augen, die einen PE innerhalb von $\pm 0,5$ dpt hatten, lag allerdings nur mit der Hill-RBF und Olsen in einem akzeptablen Bereich von über $60 \%$.

Bei den langen Augen zeigten sich insgesamt geringe MaxAE im Vergleich mit der Gesamtanalyse und der Subgruppe der kurzen Augen. Zudem erreichten, mit Ausnahme der Holladay 1 und SRK/T, alle Formeln einen Anteil von knapp 90\% innerhalb eines PE von $\pm 0,5$ dpt. Man muss allerdings beachten, dass die geringen hyperopen Fehler klinisch eher relevant sein können als myope Refraktionsfehler. 
Zusammenfassend ist zu beachten, dass die Unterschiede zwischen den einzelnen Formeln in beiden Subgruppenanalysen nicht signifikant waren. Nichtsdestotrotz scheint die Hill-RBF am besten für die IOL-Kalkulation kurzer Augen geeignet zu sein. Sollte aus Gründen der Praktikabilität eine Formel präferiert werden, die auf den Messgeräten vorinstalliert ist, so empfiehlt sich die Verwendung der Holladay 1 oder SRK/T. In der Subgruppe der langen Augen erwiesen sich die T2, Barrett Universal II und Olsen als am besten geeignet. Prinzipiell sollten die Patienten darüber aufgeklärt werden, dass die Berechnungen bei extremen AL unter Umständen ungenau sind und dass vor allem eine postoperative Hyperopie von alltäglicher Relevanz sein kann.

In jeder Subgruppe waren nur neun Augen eingeschlossen. Weitere Studien, die Formeln zur Berechnung der Linsenstärke von Tri- oder Quadrifokallinsen in extrem kurzen und langen Augen untersuchen, sollten daher durchgeführt werden. Eine Anpassung der Linsenkonstanten an die AL ist in Zukunft ebenfalls wünschenswert.

\subsubsection{Kritische Betrachtung einzelner Patienten}

Die genauen Werte jedes Auges wurden dahingehend geprüft, ob bestimmte Augen einen besonderen Einfluss auf das Resultat einzelner Formeln hatten.

Die höchsten PE bei der Verwendung aller Formeln, mit Ausnahme der Barrett Universal II, ergaben sich bei beiden Augen eines bestimmten Patienten. Das postoperative SE dieses Patienten betrug 0,00 dpt bzw. -0,25 dpt. Der Patient gab an, mit der Linse zufrieden zu sein und keine zusätzliche Korrektur für die Ferne, den Intermediärbereich oder die Nähe zu benötigen. Die Berechnungen aller Formeln lieferten $A E$ von mehr als 1,3 dpt für beide Augen. Lediglich die Barrett Universal II erzielte einen AE von 0,0 dpt bzw. 0,5 dpt. Die präoperativen Ausmessungen zeigten keine Besonderheiten. Daher ist der Grund für die Auffälligkeit bei den Berechnungen dieses Augenpaars unbekannt.

Für die schlechte Vorhersage des SE einiger Augen ließ sich keine Erklärung finden. Lediglich das Augenpaar eines Patienten wies eine Besonderheit auf. Beide Augen hatten eine kurze AL in Kombination mit einer tiefen Vorderkammer. 
In Formeln der dritten Generation wird die ACD nicht direkt eingesetzt. Stattdessen wird davon ausgegangen, dass kurze Augen grundsätzlich eine flache Vorderkammer haben. Da die Annahme bei diesem Patienten nicht erfüllt war, hätte man erwarten können, dass vor allem die Formeln der dritten Generation und die T2 unpräzise Vorhersagen liefern. Dies konnte allerdings nicht bestätigt werden. Im Gegensatz zu den Formeln, die die ACD als Parameter verwenden, wurden mit den genannten Formeln sogar bessere Ergebnisse erzielt. Die einzige Ausnahme stellte die Hill-RBF dar, bei deren Verwendung die Vorhersagen vergleichbar waren.

Kein Auge hatte einen postoperativen korrigierten Fernvisus schlechter als 0,1 logMAR. Es ist daher unwahrscheinlich, dass die Ergebnisse durch optische Beeinträchtigungen verfälscht wurden.

Da das postoperative SE durch Refraktionieren ermittelt wurde und somit eine subjektive Angabe ist, kann nicht ausgeschlossen werden, dass es falsch bestimmt wurde und im Rahmen der Berechnungen scheinbar schlechte Vorhersagen getroffen wurden. Zudem können neben intraoperativen Komplikationen bei jeder Linsenchirurgie refraktive Überraschungen auftreten. Einige Gründe sind in Tabelle 11 aufgeführt.

Tabelle 11: Gründe für refraktive Überraschungen bei der Linsenchirurgie (Tabelle verändert nach $K_{o o k^{72}}$ )

\begin{tabular}{|l|l|}
\hline Präoperativ & Postoperativ \\
\hline Schlechter Ausgangsvisus & $\begin{array}{l}\text { Hornhautödem } \\
\rightarrow \text { hyperoper Fehler }\end{array}$ \\
\hline $\begin{array}{l}\text { Okuläre Komorbiditäten, } \\
\text { z. B. Keratokonus }\end{array}$ & $\begin{array}{l}\text { Leckage/ flache Vorderkammer } \\
\rightarrow \text { hyperoper Fehler }\end{array}$ \\
\hline $\begin{array}{l}\text { Zustand nach kornealer Chirurgie, } \\
\text { z. B. keratorefraktive Chirurgie }\end{array}$ & $\begin{array}{l}\text { Zu große Kapsulorrhexis } \\
\rightarrow \text { myoper Fehler }\end{array}$ \\
\hline $\begin{array}{l}\text { Zustand nach intraokularer Chirurgie, } \\
\text { z. B. Phakovitrektomie }\end{array}$ & $\begin{array}{l}\text { Linsenverkippung } \\
\rightarrow \text { hyperoper Fehler }\end{array}$ \\
\hline $\begin{array}{l}\text { Fehler bei der Biometrie durch Komorbi- } \\
\text { ditäten, z. B. Malfixation durch Amblyopie }\end{array}$ & $\begin{array}{l}\text { Zystoides Makulaödem } \\
\rightarrow \text { hyperoper Fehler }\end{array}$ \\
\hline
\end{tabular}


Präoperative Faktoren wurden in der vorliegenden Studie durch passende Ausschlusskriterien weitgehend eliminiert. Es ist anhand des Datensatzes nicht möglich, eine Aussage zu treffen, aus welchem Grund die Berechnungen bei einigen Augen in dieser Analyse zu einem hohen PE führten.

\subsubsection{Verqleich der Sanders-Retzlaff-Kraff/theoretical mit der T2}

Bereits im Methodenteil wurden Kritikpunkte an der SRK/T aufgeführt (vergleiche 5.4.4, Seite 50). Der erstgenannte Punkt bezüglich der Berechnung der korrigierten $\mathrm{AL}$ ist nur bei extrem langen Augen zu beachten und hat daher im klinischen Altag sowie in dieser Dissertation wenig bzw. keine Relevanz. ${ }^{98}$ Bei bestimmten Konstellationen aus $\mathrm{AL}$ und K-Werten kann es allerdings zu einer Fehlberechnung kommen, die von den Autoren als "SRK/T cusp" bezeichnet wurde. ${ }^{127}$ Das Phänomen tritt bei Augen mit sehr steilen Hornhäuten auf und ist in einem Problem bei der Berechnung der Hornhauthöhe begründet. ${ }^{98}$ Eine detaillierte Tabelle der kritischen Kombinationen aus AL und K-Werten wurde 1993 publiziert. In Abbildung 15 ist ein Beispiel gezeigt. Innerhalb des markierten Bandes $(51,24 \mathrm{dpt}<\mathrm{K}$-Wert $<51,94 \mathrm{dpt})$ werden inadäquat hohe IOL-Stärken von der Formel ausgegeben. In diesem Bereich kann der Verlauf des Graphen als „Höcker" oder „Zacke“ (englisch: cusp) beschrieben werden. ${ }^{127}$

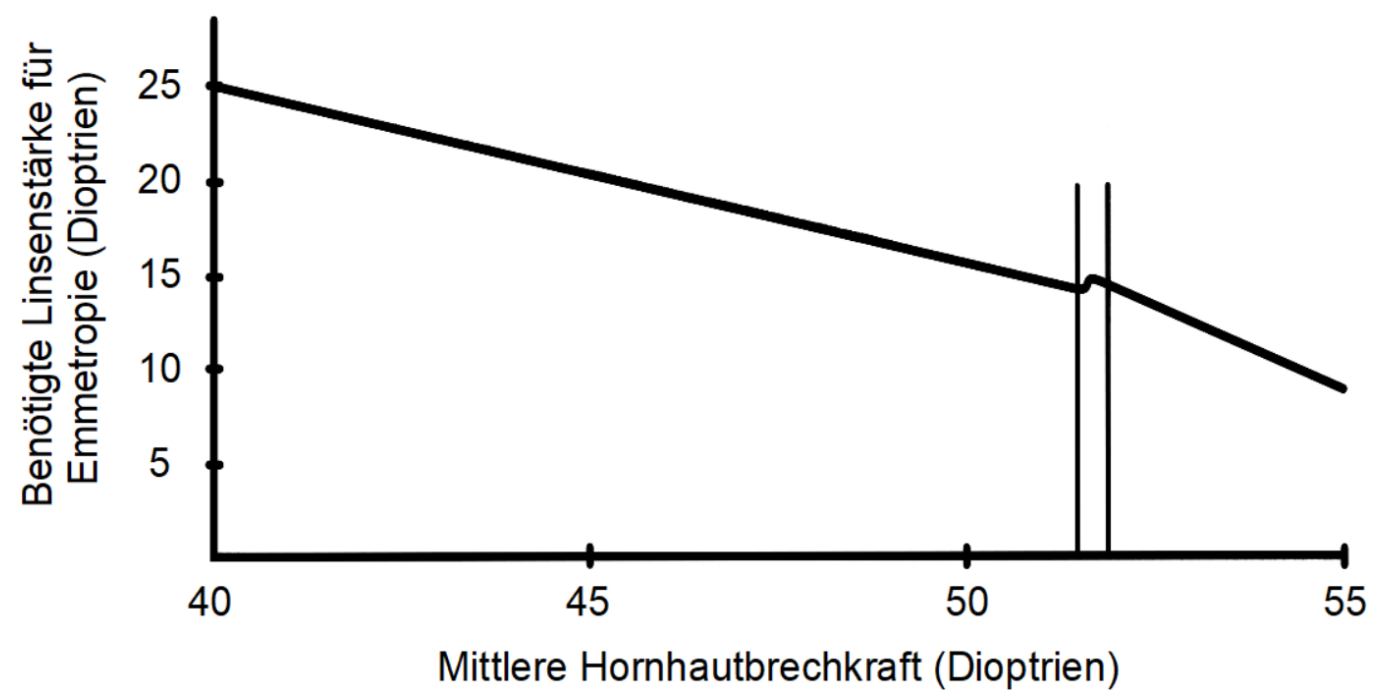

Abbildung 15: "Sanders-Retzlaff-Kraff/ theoretical cusp" bei einer Achsenlänge von $23 \mathrm{~mm}$ (Abbildung verändert nach Haigis ${ }^{127}$ ) 
Kein Auge der vorliegenden Studie wies eine Wertekonstellation auf, die innerhalb des Bandes des „SRK/T cusp“ liegen könnte, sodass alle Ergebnisse der IOL-Kalkulation mit der SKR/T als adäquat betrachtet wurden.

Die Berechnung der Hornhauthöhe wurde mit dem Erscheinen der T2 verbessert. Die Kalkulation wurde optimiert und das Phänomen des "SRK/T cusp“ eliminiert. ${ }^{98}$ Analog zu anderen Studien ergab sich in der vorliegenden Analyse ein geringerer MAE der T2 im Vergleich zur SRK/T. 12,120,121 Der Unterschied war allerdings nach der Bonferroni-Korrektur nicht statistisch signifikant (WilcoxonVorzeichen-Rang-Test: $p=0,03$ ). Die Verwendung der T2 wird dadurch eingeschränkt, dass sie bisher nicht auf gängigen Biometern vorinstalliert ist. Eine Computersoftware existiert ebenfalls noch nicht. Es gibt lediglich ein ExcelDatenblatt, mit dem die Berechnung durchgeführt werden kann. Die Optimierung der A-Konstanten gestaltet sich daher problematisch. Es ist unwahrscheinlich, dass die Vorgehensweise dieser Dissertation, d. h. die Verwendung der Waswäre-wenn-Analyse mit Zielwertanalyse, im klinischen Alltag umgesetzt wird. Die Autoren der T2 deklarieren allerdings, dass eine bereits optimierte A-Konstante der SRK/T problemlos für die T2 verwendet werden kann. ${ }^{98} \mathrm{Um}$ diese These zu überprüfen, wurden die Berechnungen mit der T2 erneut unter Verwendung der für die $\mathrm{SRK} / \mathrm{T}$ optimierten A-Konstanten durchgeführt. Der Unterschied der beiden A-Konstanten betrug 0,064. Der MAE der T2 war mit der optimierten SRK/T-Konstanten nur um 0,002 dpt höher als mit der optimierten T2-Konstanten (Wilcoxon-Vorzeichen-Rang-Test: $p=0,55$ ). Der MedAE war sogar etwas geringer ( 0,23 dpt vs. 0,25 dpt) und der Anteil der Augen mit einem PE innerhalb von $\pm 0,25$ dpt höher (53,3\% vs. 50,7\%). Verglichen mit dem MAE der SRK/T war der MAE der T2 mit der SRK/T-Konstanten geringer (0,35 dpt vs. 0,39 dpt). Dieser Unterschied war jedoch ebenfalls nicht signifikant (Wilcoxon-VorzeichenRang-Test: $p=0,10)$.

Zusammenfassend kann man sagen, dass die T2 der SRK/T vorgezogen werden sollte. Für den Fall, dass die T2-Konstante nicht optimiert werden kann, stellt die Verwendung der SRK/T-Konstanten für Berechnungen mit der T2 eine gute Möglichkeit dar. 


\subsubsection{Besondere Aspekte der Hill-radiale Basisfunktion}

Die Hill-RBF ist eine Formel, die einen völlig neuen Ansatz verfolgt. Im Gegensatz zu den herkömmlichen Formeln basiert sie nicht auf optischen Prinzipien, sondern auf Mustererkennung und Dateninterpolation.

Sie arbeitet mit einem integrierten Grenzmodell, das nur dann zuverlässige Ausgaben produziert, wenn die Augenausmessungen innerhalb eines bestimmten Bereichs liegen. Fünf Augen der vorliegenden Studie wurden als "out of bounds" deklariert. Nichtsdestotrotz wurden sie bei der Analyse nicht ausgeschlossen. Die Hill-RBF hat insgesamt gut abgeschnitten, obwohl Augen eingeschlossen waren, für die gegebenenfalls unzuverlässige Vorhersagen getroffen wurden.

Die Formel wurde basierend auf Datensätzen von Patienten entwickelt, deren Messungen mit dem Lenstar erfolgten und die eine monofokale AcrySof IQ SN60WF IOL implantiert bekamen. Trotzdem soll sie auch für andere Biometer und IOL zuverlässig anwendbar sein. ${ }^{137}$ Die Metaanalyse von Huang et al zeigte, dass die Messwerte des Lenstars mit denen des IOLMasters vergleichbar sind. ${ }^{161}$ Ein signifikanter Unterschied wurde nur bei Messungen des WTW gefunden. Da dieser Wert kein obligater Parameter bei den Berechnungen der Hill-RBF ist, wird vermutet, dass die Nutzung des IOLMasters im Rahmen dieser Dissertation keinen Einfluss auf die Bewertung der Formel hatte.

Mit Ausnahme des MaxAE war die Hill-RBF in jeder Kategorie unter den besten drei Formeln. Da sie auf künstlicher Intelligenz beruht und daher permanent durch die eingegebenen Daten weiterentwickelt wird, ist zu erwarten, dass sich die Ergebnisse mit dieser Formel in Zukunft noch weiter verbessern werden.

\subsection{Einordnung in den aktuellen Wissensstand}

Es existieren bereits zahlreiche, teils sehr große Studien, in denen Kalkulationsformeln für die Berechnung der Linsenstärke von Monofokallinsen evaluiert wurden. ${ }^{112,120,140}$ Beim Vergleich der neuesten Formeln für die Berechnung von Monofokallinsen schnitt die Kane am besten ab, gefolgt von der Olsen, Barrett Universal II, EVOF und Hill-RBF. Schlechtere Ergebnisse wurden mit den 
Standardformeln Holladay 1, SRK/T, Hoffer Q, Holladay 2 und Haigis erzielt. ${ }^{162}$ Dies zeigt, dass es lohnenswert und wichtig ist, weiterhin neue Formeln zu entwickeln, diese mit den bewährten Formeln zu vergleichen und die Vorteile im klinischen Alltag für das Wohl des Patienten zu nutzen.

\subsubsection{Linsenkalkulationsformeln für monofokale Linsen}

Die vorliegende Studie evaluierte explizit verschiedene Formeln im Zusammenhang mit einer Quadrifokallinse. Daher soll die Diskussion der Studien mit monofokalen Linsen auf eine große Publikation von Melles et al beschränkt werden. Die übrigen in diesem Abschnitt diskutierten Arbeiten analysierten Ergebnisse der Hill-RBF. Die Anwendung dieser neuen Formel für Multifokallinsen wurde bislang in keiner Studie untersucht.

Melles et al publizierten 2018 eine Studie, in der über 18.000 Augen eine AcrySof IQ SN60WF- oder SA60AT-Monofokallinse implantiert bekamen. ${ }^{140}$ Mit Ausnahme der T2 und Hill-RBF evaluierten sie die Formeln, die auch in der vorliegenden Analyse eingeschlossen waren. Im Gegensatz zu dieser Dissertation wurden die präoperativen Messungen mit dem Lenstar vorgenommen. Die Vorhersagefähigkeit der IOL-Kalkulationsformeln ist jedoch unter Verwendung der Messwerte des Lenstars und derer des IOLMasters vergleichbar. ${ }^{81,82}$ Das Durchschnittsalter der Patienten war knapp zehn Jahre höher als das in der vorliegenden Arbeit. Die Bestimmung der postoperativen Refraktion erfolgte bereits nach vier Wochen und könnte daher in einigen Fällen nicht den endgültigen Zustand darstellen. Melles et al fanden bessere Ergebnisse bei Berechnungen mit der Barrett Universal II im Vergleich zur Holladay 1, SRK/T, Hoffer Q, Holladay 2, Haigis und Olsen. Alle Unterschiede waren im WilcoxonVorzeichen-Rang-Test nach der Bonferroni-Korrektur signifikant. ${ }^{140}$ Der MAE der Barrett Universal II war mit 0,31 $\pm 0,40 \mathrm{dpt}$ (SN60WF) bzw. 0,32 $\pm 0,42 \mathrm{dpt}$ (SA60AT) etwas höher als in der vorliegenden Analyse (0,29 $\pm 0,31 \mathrm{dpt})$. Die Ergebnisse bei Berechnungen mit der Olsen waren mit einem MAE von 0,33 \pm 0,42 dpt (SN60WF) und 0,34 $\pm 0,44 \mathrm{dpt}$ (SA60AT) vergleichbar mit den Ergebnissen dieser Dissertation $(0,34 \pm 0,45 \mathrm{dpt})$, wobei allerdings die vorinstallierte Version auf dem Lenstar statt des PhacoOptics-Programms genutzt wurde. Die 
Haigis war mit einem MAE von 0,34 $\pm 0,44$ dpt (SN60WF) bzw. 0,35 $\pm 0,45 \mathrm{dpt}$ (SA60AT) die drittbeste Formel in der Analyse von Melles et al. ${ }^{140}$ In der vorliegenden Studie wurde mit ihr mit 0,38 $\pm 0,51 \mathrm{dpt}$ ein höherer MAE als in der genannten Publikation erreicht. Mit Ausnahme der Hoffer $Q$ waren die MedAE aller Formeln höher als die dieser Dissertation. Die Prozentzahlen der Augen, deren $P E$ innerhalb von $\pm 0,50$ dpt lagen, waren vergleichbar. Für die SRK/T, Holladay 2, Haigis, Barrett Universal II und Olsen konnten in der vorliegenden Arbeit größere Anteile an Augen mit einem PE innerhalb von $\pm 0,25 \mathrm{dpt}$ verzeichnet werden, während Prozentzahlen mit einem PE innerhalb von $\pm 1,0$ dpt mit allen Formeln niedriger waren. Insgesamt ist das Resultat von Melles et al hinsichtlich der guten Vorhersagefähigkeit der Barrett Universal II und Olsen mit den Ergebnissen dieser Dissertation vereinbar. Die Haigis schnitt in der vorliegenden Analyse schlechter ab.

Eine Untersuchung von Kane et al an mehr als 3.000 Augen ergab, dass die Berechnungen mit der Hill-RBF signifikant schlechter waren als mit der Holladay 1 und Barrett Universal II. ${ }^{121}$ Dies konnte in der vorliegenden Studie nicht bestätigt werden, obwohl die Augen, die als "out of bounds" deklariert wurden, im Gegensatz zur Analyse von Kane et al, nicht ausgeschlossen wurden und somit tendenziell mehr unzuverlässige Vorhersagen mit der Hill-RBF getroffen wurden. Der MAE war in dieser Dissertation mit 0,33 dpt niedriger als der MAE der genannten Publikation mit 0,41 dpt. Die Ergebnisse der Hill-RBF waren vergleichbar mit denen der Barrett Universal II und besser als die der Holladay 1. Etwa $70 \%$ der Augen hatten in der Studie von Kane et al bei Berechnungen mit der Haigis, Barrett Universal II und Hill-RBF einen PE innerhalb von $\pm 0,5$ dpt. $^{121}$ In der vorliegenden Analyse lag dieser Wert bei 80,0\%.

Bei der Berechnung der Stärke einer Monofokallinse, die im Rahmen einer Femtosekundenlaser-assistierten Kataraktoperation implantiert wurde, fanden Roberts et al keine signifikanten Unterschiede zwischen der SRK/T, Hoffer Q, Holladay 2, Barrett Universal II und Hill-RBF. Die MAE schwankten zwischen $0,30 \mathrm{dpt}$ und 0,34 dpt. Die beiden letztgenannten Formeln lieferten sowohl für kurze als auch für lange Augen gute Ergebnisse. Insgesamt wurden die exaktesten Ergebnisse mit der Barrett Universal II erreicht, was sich mit den Erkenntnissen dieser Dissertation deckt. ${ }^{163}$ In der vorliegenden Arbeit erzielte die 
Hill-RBF allerdings exaktere Vorhersagen im Vergleich mit der SRK/T, Hoffer $Q$ und Holladay 2.

In der vorliegenden Studie, die bereits im August 2018 publiziert wurde, wurde erstmals die Version 2.0 der Hill-RBF evaluiert. Im Jahr 2019 schlossen Melles et al aus den Erkenntnissen von zwei eigenen Untersuchungen, dass die Version $2.0 \mathrm{im}$ Vergleich zur Version 1.0 exaktere Vorhersagen für Monofokallinsen liefert. Im Gegensatz zu dieser Dissertation waren in ihrer Analyse die Resultate der Hill-RBF trotzdem schlechter als die der Barrett Universal II und Olsen. ${ }^{162}$

Wallace et al verglichen die SRK/T, Barrett Universal II und Hill-RBF 2.0. Ebenso wie in der vorliegenden Arbeit wurde der IOLMaster 500 für präoperative Messungen verwendet. Die Unterschiede der AE waren nach drei Monaten nicht signifikant. ${ }^{164}$ Dieses Resultat stimmt nicht mit der vorliegenden Analyse überein, in der die Vorhersagefähigkeit der SRK/T signifikant schlechter war als die der beiden anderen Formeln. Die MAE aller drei Formeln waren wesentlich höher als in der vorliegenden Studie (SRK/T: 0,52 dpt vs. 0,39 dpt; Barrett Universal II: 0,50 dpt vs. 0,29 dpt; Hill-RBF: 0,49 dpt vs. 0,33 dpt). Die Anteile der Augen mit einem PE innerhalb von $\pm 0,25 \mathrm{dpt}, \pm 0,5 \mathrm{dpt}$ und $\pm 1,0 \mathrm{dpt}$ waren deutlich geringer. ${ }^{164}$

Wan et al führten eine Untersuchung an Augen mit einer $A L$ von mindestens 26 mm durch. Die Hill-RBF 2.0 war den Formeln der dritten Generation überlegen und vergleichbar mit der Haigis und Barrett Universal II. ${ }^{165}$ In der vorliegenden Analyse gab es in der Subgruppe der langen Augen keinen signifikanten Unterschied zwischen den Formeln. Die ermittelten SD waren für alle Formeln verglichen mit den Werten von Wan et al niedriger, ebenso wie die MedAE der Hoffer Q, Haigis, Barrett Universal II und Hill-RBF.

Zusammenfassend kann man festhalten, dass die Hill-RBF in den bisher erschienenen Studien an Monofokallinsen nicht überzeugen konnte. In dieser Dissertation war die Vorhersagefähigkeit der neuen Formel besser als die der Holladay 1, SRK/T, Hoffer Q und Holladay 2. Sie war zudem nicht schlechter als die der übrigen Formeln. Die Hill-RBF erwies sich daher für die Linsenkalkulation der Quadrifokallinse als geeignet. 


\subsubsection{Linsenkalkulationsformeln für multifokale Linsen}

Reitblat et al untersuchten die Exaktheit der Berechnungen der Stärke einer Bifokallinse mit der Holladay 1, Hoffer Q, SRK/T, Haigis, Olsen, Holladay 2 und Barrett Universal II. Die präoperativen Messungen der 73 Augen wurden mit dem IOLMaster 500 und dem Lenstar vorgenommen. Die Berechnungen der Olsen erfolgten mit dem Lenstar. Das Durchschnittsalter der Patienten entsprach dem der vorliegenden Studie. Im Gegensatz zu dieser Dissertation wurde die Operation hingegen nicht Femtosekundenlaser-assistiert durchgeführt. Zudem war das postoperative SE mit $-0,33 \pm 0,28$ dpt höher im Vergleich zu dem der vorliegenden Arbeit mit $-0,02 \pm 0,35 \mathrm{dpt}$. Die optimierten Linsenkonstanten aller Formeln waren höher. Keine Formel war bezüglich der Vorhersagefähigkeit besser als andere. Außerdem konnte kein Unterschied zwischen den Messgeräten nachgewiesen werden. Die MAE mit dem IOLMaster reichten von $0,22 \mathrm{dpt}$ mit der Holladay 2 bis 0,25 dpt mit der Hoffer $Q$ und Haigis. Sie waren ebenso wie die SD (Spannweite: 0,16 bis 0,19 dpt) und die MedAE (Spannweite: 0,17 bis $0,21 \mathrm{dpt}$ ) wesentlich geringer als die in der vorliegenden Studie ermittelten Werte. ${ }^{82}$ Lediglich die MedAE der Haigis und Barrett Universal II waren in dieser Dissertation mit 0,21 dpt bzw. 0,20 dpt vergleichbar niedrig. Mit Ausnahme der Barrett Universal II wurden in der Studie von Reitblat et al deutlich höhere Anteile an Augen mit einem PE innerhalb von $\pm 0,25 \mathrm{dpt}$ und $\pm 0,5 \mathrm{dpt}$ erreicht. Keine Formel war jedoch für die Linsenkalkulation der Bifokallinse einer anderen Formel überlegen. ${ }^{82}$

Ramji et al konnten beim Vergleich der Holladay 1, SRK/T, Hoffer Q, Holladay 2 und Haigis an 116 Augen ebenfalls keine Unterschiede zwischen den Formeln feststellen. Die SD bei den Berechnungen der Linsenstärke einer Bifokallinse waren, mit Ausnahme der SRK/T, vergleichbar mit den Ergebnissen der vorliegenden Analyse. Die MaxAE waren für alle Formeln, ebenfalls mit Ausnahme der $\mathrm{SRK} / \mathrm{T}$, wesentlich niedriger. Die Vergleichbarkeit mit dieser Dissertation wurde zum einen dadurch eingeschränkt, dass die MAE und MedAE nicht angegeben wurden, und zum anderen, dass eine Optimierung der Linsenkonstanten nicht für alle Formeln durchgeführt wurde. In der Subgruppe der Augen mit einer AL von weniger als $22 \mathrm{~mm}$ wurde mit einem ME von $-0,33 \pm 0,41 \mathrm{dpt}$ bzw. 0,36 $\pm 0,38 \mathrm{dpt}$ ein signifikanter Unterschied zwischen der SRK/T und Haigis festgestellt. ${ }^{166}$ Aus 
klinischer Sicht ist der myope Fehler bei Berechnungen der SRK/T eher tolerabel. Diese Formel sollte daher der Haigis vorgezogen werden, was durch die Erkenntnisse der vorliegenden Subgruppenanalyse bestätigt wird, in der die SRK/T in allen Bereichen besser als die Haigis abschnitten hat.

Eine 2019 publizierte Studie von Kim et al evaluierte die visuellen Ergebnisse nach Implantation einer bifokalen oder trifokalen Linse. Ein Teil der Untersuchung bestand aus der Beurteilung der folgenden IOL-Kalkulationsformeln: Holladay, SRK/T, Hoffer Q, Haigis und Barrett Universal II. Ob die Holladay 1 oder 2 genutzt wurde, ist der Publikation nicht zu entnehmen. 28 Augen erhielten eines von zwei bifokalen Modellen. 53 Augen bekamen eine Trifokallinse implantiert. ${ }^{167}$ Da keine Optimierung der Linsenkonstanten stattfand, ist ein Vergleich der MAE mit denen der vorliegenden Studie nicht zielführend. Die SD reichten in der Trifokallinsen-Gruppe von 0,20 dpt bis 0,23 dpt und waren somit deutlich niedriger als in dieser Dissertation mit einer minimalen SD von 0,31 dpt bei Verwendung der Barrett Universal II. Gleiches galt für die SD der beiden Bifokallinsen-Gruppen. MaxAE oder Prozentzahlen an Augen mit einem bestimmten PE wurden nicht berichtet. Obwohl in keiner Gruppe ein signifikanter Unterschied zwischen den Formeln nachgewiesen wurde, zeigte sich vor allem für die Bifokallinsen eine geringfügige Überlegenheit der Haigis und Barrett Universal II. ${ }^{167}$ Dies deckt sich mit den vorliegenden Ergebnissen insofern, als dass die Barrett Universal II die Formel ist, die in der Zusammenschau aller Parameter am besten für die Berechnung der Quadrifokallinse geeignet ist.

Insgesamt lassen sich aus den bisherigen Publikationen zu Multifokallinsen keine eindeutigen Schlüsse ziehen, da die Unterschiede zwischen den einzelnen Formeln nicht signifikant waren. Eine konsequente Optimierung der Linsenkonstanten, die zur Reduktion systematischer Fehler erforderlich ist, wurde nur in der Arbeit von Reitblat et al durchgeführt. ${ }^{82}$ Zudem sind die Fallzahlen der bisher publizierten Untersuchungen vergleichsweise gering. Um die Ergebnisse dieser Dissertation zu bestätigen und klare Empfehlungen im Zusammenhang mit Bi- und Trifokallinsen auszusprechen, sind daher größere Studien mit einem einheitlichen Vorgehen, wie es von Wang et al vorgeschlagen wurde, erforderlich. ${ }^{139}$ 


\subsubsection{Problematik der Augen mit extremen Maßen}

Es gibt keine „Universalformel“, die in jeder Situation und bei jedem Patienten hervorragende Ergebnisse liefert. Regressionsformeln sind prinzipiell für extrem kurze oder lange Augen weniger geeignet, da sie auf empirischen Analysen von durchschnittlichen Datensätzen beruhen. ${ }^{55}$

\subsubsection{Extreme Achsenlänge}

Die Grenzwerte für die Klassifizierung der Augen als „extrem kurz“ oder „extrem lang" werden je nach Publikation unterschiedlich gewählt. In dieser Dissertation wurde die Einteilung einer 2018 in Ophthalmology erschienenen Publikation von Melles et al übernommen. ${ }^{140}$ Die Gruppe der kurzen Augen umfasste Augen mit einer $\mathrm{AL}<22,5 \mathrm{~mm}$, die Gruppe der langen Augen solche mit einer $\mathrm{AL}$ $>25,5 \mathrm{~mm}$.

Je nach AL des Auges sind bestimmte Formeln besser geeignet als andere. In einer Leitlinie des National Institute for Health and Care Excellence, die 2017 erschien, wurden auf der Basis von 17 Studien die in Tabelle 12 dargestellten Empfehlungen ausgesprochen. ${ }^{160} \mathrm{Es}$ ist zu beachten, dass in die Bewertung die Holladay 1, T2 und Hill-RBF nicht einbezogen wurden, sowie dass die Empfehlungen unabhängig vom implantierten Linsentyp gelten.

Tabelle 12: Empfohlene Formeln bei unterschiedlichen Achsenlängen (Quelle: National Institute for Health and Care Excellence ${ }^{160}$ )

\begin{tabular}{|l|l|}
\hline Achsenlänge & Empfohlene Formeln \\
\hline$<22 \mathrm{~mm}$ & Haigis oder Hoffer Q \\
\hline $22-26 \mathrm{~mm}$ & Barrett Universal II (alternativ: SRK/T) \\
\hline$>26 \mathrm{~mm}$ & Haigis oder SRK/T \\
\hline
\end{tabular}

Aufgrund der Ergebnisse der vorliegenden Analyse sollten die Angaben der Leitlinie im Zusammenhang mit der PanOptix kritisch gesehen werden. Die Hoffer $Q$ und Haigis lieferten in kurzen Augen schlechtere Vorhersagen als die anderen Formeln. In langen Augen erwies sich die Verwendung der SRK/T oder Haigis ebenfalls als nicht empfehlenswert. 
Formeln mit zwei Variablen, deren ELP-Vorhersagen lediglich auf der AL und den K-Werten beruhen, sind für kurze oder lange Augen nur bedingt geeignet, da sich die ACD häufig nicht - wie von diesen Formeln angenommen - linear zur AL verhält. Aus diesem Grund ist zu erwarten, dass die Formeln, die die präoperative ACD direkt in die Vorhersage der Implantatposition miteinbeziehen, exaktere Vorhersagen liefern. ${ }^{168}$ In dieser Dissertation wurden jedoch auch mit der Holladay 1, SRK/T und T2 gute Ergebnisse bei kurzen Augen erzielt.

Wang et al publizierten zwei Metaanalysen, in denen sie jeweils untersuchten, welche Formeln für extreme $\mathrm{AL}$, unabhängig vom Linsentyp, geeignet sind. Beim Vergleich der Holladay 1, SRK/T, Hoffer Q, Holladay 2 und Haigis stellte sich heraus, dass die Haigis für hyperope Augen mit einer $A L<22 \mathrm{~mm}$ am besten geeignet ist. ${ }^{169}$ Dies konnte in der vorliegenden Studie nicht bestätigt werden. In der Subgruppenanalyse der kurzen Augen schnitt die Haigis am schlechtesten ab. Bei der IOL-Kalkulation für lange Augen mit einer $\mathrm{AL}>24,5 \mathrm{~mm}$ war die Barrett Universal II in der Metaanalyse von Wang et al im Vergleich zu den Formeln der dritten Generation sowie der Holladay 2 und Haigis überlegen. ${ }^{170}$ Diese Erkenntnis stimmt mit den Ergebnissen dieser Dissertation überein.

In einer Studie an 51 myopen Augen mit einer $A L>25 \mathrm{~mm}$ war die Genauigkeit der Hill-RBF besser als die der Holladay 1 und SRK/T. Zwischen der Hill-RBF und Holladay 2 bzw. Barrett Universal II zeigte sich kein Unterschied. ${ }^{171}$ In der vorliegenden Analyse wurden ähnliche Ergebnisse festgestellt. Die Barrett Universal II war bei langen Augen vergleichbar mit der T2 und Olsen.

Melles et al beobachteten die besten Ergebnisse bei der Berechnung für kurze Augen mit der Barrett Universal II und für lange Augen mit der Olsen. Die Hoffer schien für extrem kurze oder lange Augen ungeeignet. Die Barrett Universal II war am wenigsten anfällig für abnorme Augenausmessungen, wie extreme $A L$, K-Werte und ACD. ${ }^{140}$ Aufgrund hervorragender Ergebnisse auch bei außergewöhnlichen Augen empfohlen Melles et al die Verwendung der Kane, Olsen oder Barrett Universal II unabhängig von der AL. ${ }^{162}$ Die Olsen und Barrett Universal II zeigten sich auch in der vorliegenden Analyse als am besten geeignet für die IOL-Kalkulation von myopen Augen. Bei der Berechnung von hyperopen Augen ergaben sich allerdings bessere Ergebnisse mit der Hill-RBF. 
Da es bei der IOL-Kalkulation von langen Augen häufig zu hyperopen Refraktionsfehlern kommt, wurden von Wang et al Möglichkeiten entwickelt, die gemessene $A L$ für Berechnungen mit der Holladay 1, SRK/T, Hoffer $Q$, Holladay 2 und Haigis zu optimieren. ${ }^{172,173}$ In einer Studie von Melles et al wurde diese Wang-Koch-Anpassung kritisch diskutiert, da sie zu einer Überkorrektur in Richtung myoper Fehler führte. ${ }^{140}$ In dieser Dissertation wurde die Anpassung nicht angewandt. Das Ziel war, die ursprünglichen Formeln zu analysieren, wie sie z. B. auch auf den Biometriegeräten verfügbar sind.

Insgesamt bleibt festzuhalten, dass für besonders kurze oder lange Augen keine eindeutigen Empfehlungen vorliegen. Besonders für die Berechnung der Linsenstärke von Multifokallinsen sind daher weitere große Studien notwendig.

\subsubsection{Extreme Keratometriewerte oder Vorderkammertiefen}

Neueste Forschungsansätze beschäftigen sich mit der Auswahl der Formeln abhängig von der Hornhautbrechkraft oder der ACD.

Reitblat et al untersuchten verschiedene Formeln in Augen mit besonders flachen oder steilen Hornhäuten. Zusätzlich zur generellen Empfehlung, in diesen Fällen die Formeln der neueren Generationen zu verwenden, wird eine Anpassung der SRK/T je nach Hornhautbrechkraft vorgeschlagen. ${ }^{102}$ In einer anderen Studie zeigte die SRK/T eine starke Fehleranfälligkeit gegenüber extremen K-Werten. Flache Hornhäute führten zu einem deutlich hyperopen Refraktionsfehler, steile Hornhäute hingegen zu einer Myopie. ${ }^{140}$

Gökce et al evaluierten acht Formeln in Augen mit normaler AL, aber sehr tiefer oder flacher Vorderkammer. ${ }^{168} \mathrm{Da}$ diese Besonderheit sowohl mit einer irregulären Anatomie als auch mit einer verhältnismäßig dicken oder dünnen kristallinen Linse zusammenhängen kann, liegt es nahe, die Formeln zu nutzen, die die Implantatposition unter Einbezug der LT vorhersagen. Nichtsdestotrotz stellte sich die Barrett Universal II bei extremen ACD als die am besten geeignete Formel heraus, obwohl die LT bei der Berechnung nicht beachtet wird. ${ }^{168}$

Aufgrund der geringen Fallzahl der vorliegenden Studie erschien es nicht sinnvoll, Subgruppenanalysen für extreme K-Werte oder ACD durchzuführen. 


\subsection{Verbesserung der visuellen Ergebnisse mit multifokalen Linsen durch die Verwendung geeigneterer Formeln}

Eine Umfrage der European Society of Cataract and Refractive Surgery aus dem Jahr 2017 ergab, dass drei Viertel der über 1.900 Befragten die altbewährte SRK/T präferieren (siehe Abbildung 16). Zwei Drittel gaben an, dass sie bei der Einführung einer neuen Linse ihre persönlichen Linsenkonstanten optimieren. ${ }^{174}$ In der Umfrage wurde nicht differenziert, welche Formeln für welchen Linsentyp verwendet werden. Es liegt jedoch nahe, dass die SRK/T aufgrund der großen Beliebtheit auch für die Kalkulation von Multifokallinsen genutzt wird.

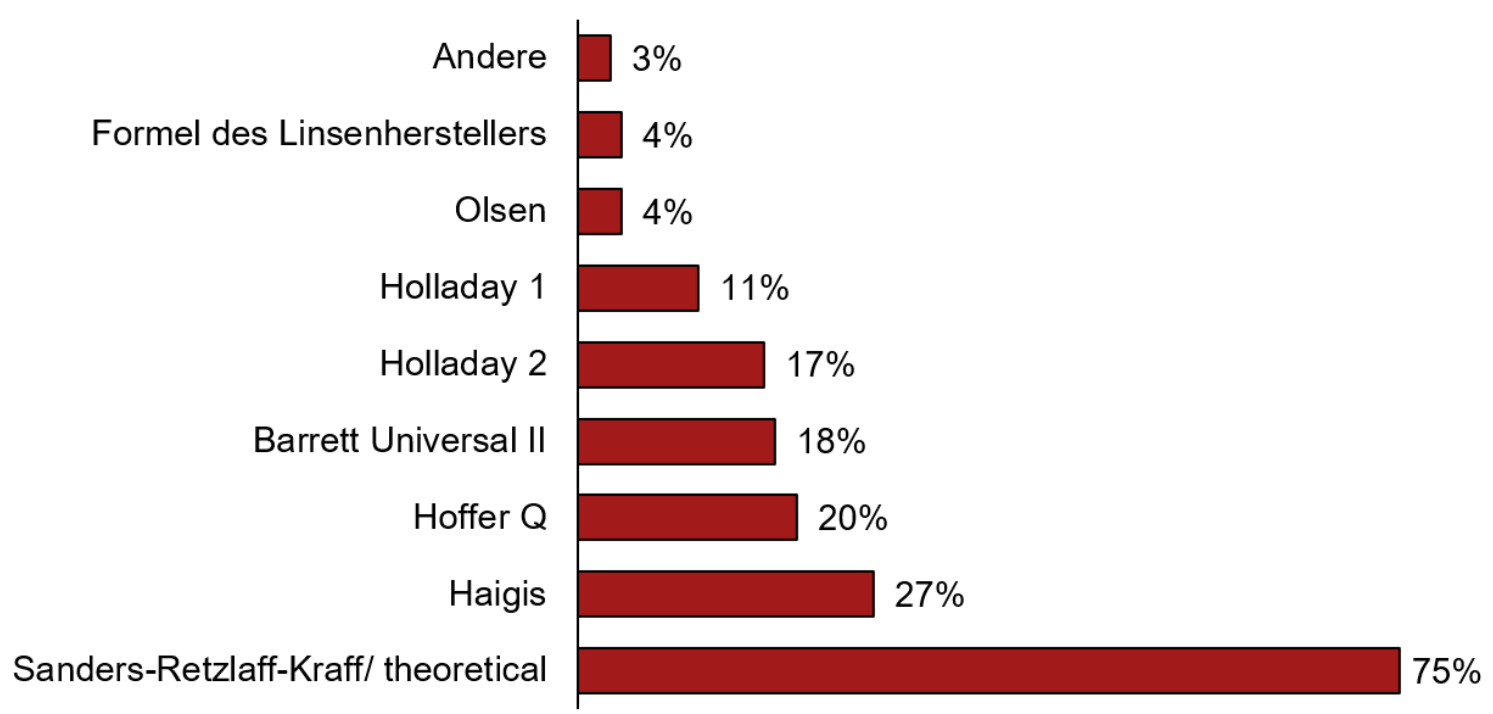

Abbildung 16: Bevorzugt verwendete Formeln laut Umfrage der European Society of Cataract and Refractive Surgery

(Abbildung verändert nach European Society of Cataract and Refractive Surgery ${ }^{174}$ )

Die visuellen Ergebnisse nach Implantation der quadrifokalen PanOptix wurden bereits in einigen Studien untersucht. ${ }^{175-180}$ In keiner der Arbeiten wurden die Linsenkonstanten zuvor optimiert. In der Studie von Mencucci et al wurde in Abhängigkeit von der AL die Hoffer Q oder Holladay 1 verwendet. Die 40 Augen hatten ein postoperatives SE von $-0,20 \pm 0,56$ dpt. $^{179}$ In zwei Arbeiten von Monaco et al und Martínez de Carneros-Llorente et al erfolgte die IOL-Kalkulation von jeweils 40 Augen mit der SRK/T. Der Unterschied zwischen dem erwarteten und erreichten SE der erstgenannten Publikation betrug $-0,21 \pm 0,04$ dpt. ${ }^{176}$ Das 
postoperative SE der zweiten Studie war -0,03 \pm 0,16 dpt (Spannweite: $-0,62$ bis $0,75 \mathrm{dpt}) .{ }^{178}$ Akman et al nutzten die Holladay 2 zur Berechnung der Linsenstärke von 96 Augen. Das postoperative SE wurde mit 0,73 $\pm 0,31 \mathrm{dpt}$ angegeben. ${ }^{177}$ Kohnen et al berechneten die Linsenstärke der PanOptix mit der Haigis. Das erreichte postoperative SE der 54 Augen betrug $-0,04 \pm 0,32 \mathrm{dpt}$ (Spannweite: $-1,00$ bis $1,13 \mathrm{dpt}$ ). Nach drei Monaten hatten $93 \%$ der Augen ein SE innerhalb von $\pm 0,5$ dpt. ${ }^{175}$ In der Studie von Lawless et al wurden die Holladay 2 und Barrett Universal II zur IOL-Kalkulation herangezogen. Es wurde allerdings nicht differenziert, für wie viele Augen welche Formel verwendet wurde. ${ }^{180}$

Mit Ausnahme der letztgenannten Arbeit wurden in allen bisher publizierten Studien Formeln genutzt, die laut der vorliegenden Analyse nicht für die Kalkulation der PanOptix empfohlen werden können. Es ist folglich zu erwarten, dass die Ergebnisse nach der Implantation der quadrifokalen Linse besser sein könnten, wenn statt den altbewährten Formeln geeignetere Formeln mit optimierten Linsenkostanten verwendet werden würden. Besonders das hohe SE in der Studie von Akman et al ist aus klinischer Sicht relevant und lässt sich gegebenenfalls auf die Verwendung der Holladay 2 zurückführen.

\subsection{Limitationen}

Die bereits erörterten Limitationen der vorliegenden Studie sind im Folgenden zusammenfassend genannt.

Die LT konnte mit dem IOLMaster 500 nicht gemessen und somit auch nicht für die Berechnungen verwendet werden (vergleiche 7.1.3, Seite 73).

Die Wang-Koch-Anpassung für lange Augen wurde nicht angewandt (vergleiche 7.3.3.1, Seite 91).

Die Studienpopulation ist relativ klein. Mit Ausnahme eines Patienten wurden daher von allen Patienten beide Augen eingeschlossen. Eine Anpassung der statistischen Auswertung wurde nicht vorgenommen (vergleiche 7.1.1, Seite 70). Aufgrund der geringen Fallzahl ist die Subgruppenanalyse für extreme AL nur begrenzt aussagekräftig (vergleiche 7.2.3, Seite 80). 


\subsection{Schlussfolgerungen und Ausblick}

Experience is simply the name we give our mistakes. ${ }^{D}$

(Oscar Wilde)

Fehler wurden gemacht und Fehler werden weiterhin passieren. Wichtig ist, dass aus diesen Fehlern die entsprechenden Folgerungen abgeleitet werden. Das Hauptziel aller therapeutischen Maßnahmen sollte immer die größtmögliche Patientenzufriedenheit sein. Eine Verwendung ungeeigneter Linsenkalkulationsformeln, die zu unerwünschten postoperativen Ergebnissen führen, sollte daher unbedingt vermieden werden.

Es gibt trotz zahlreicher Studien immer noch ungelöste Probleme, wie beispielsweise die exakte Vorhersage der Linsenposition oder das Auftreten optischer Phänomene nach der Implantation von Multifokallinsen. Für beide Aspekte existieren vielversprechende Ansatzpunkte in der klinischen Forschung, die weiterverfolgt werden sollten. Die Entwicklung der refraktiven Kataraktchirurgie ist noch lange nicht abgeschlossen.

Ausgehend von den Erkenntnissen der vorliegenden Arbeit sollte angestrebt werden, die aktuell verwendeten Linsenformeln kritisch zu hinterfragen und sich gegebenenfalls von altbewährten Formeln zu lösen. Insbesondere im Zusammenhang mit Multifokallinsen scheint es lohnend, die Vorhersageexaktheit der modernen Formeln zu evaluieren und diese in Zukunft für die IOL-Kalkulation in Betracht zu ziehen. Mit der Verwendung neuerer Formeln können exaktere Ergebnisse erzielt werden. Dies ist insbesondere für die Patienten relevant, die sich für eine Premiumlinse entscheiden und hohe Erwartungen haben.

Diese Studie ist die erste, die Linsenkalkulationsformeln für die Berechnung einer Quadrifokallinse untersuchte. Die Barrett Universal II lieferte die präzisesten Vorhersagen der postoperativen Refraktion. Die T2, Olsen und Hill-RBF führten ebenfalls zu niedrigen Vorhersagefehlern. Es wird empfohlen, eine dieser Formeln künftig für die Berechnung der Linsenstärke der quadrifokalen PanOptix

\footnotetext{
D Übersetzung: Erfahrung - so nennen wir einfach unsere Fehler
} 
zu nutzen. Weitere Studien sind notwendig, um die Resultate dieser Dissertation in größeren Patientengruppen zu bestätigen. Zudem sollten sehr neue Formeln, wie z. B. die Kane oder EVOF, im Zusammenhang mit Multifokallinsen evaluiert werden.

Eine wichtige Frage, die die vorliegende Arbeit nicht zuverlässig beantworten konnte, ist, welche Formeln bei extremen Augenmaßen am besten geeignet sind. Dies erfordert Studien mit einer ausreichenden Anzahl an kurzen und langen Augen. Eine Evaluation der Kalkulationsformeln entsprechend der Hornhautbrechkraft und ACD erscheint ebenfalls sinnvoll. 


\section{Zusammenfassung}

Hintergrund und Ziel der Studie:

Steigende Erwartungen der Kataraktpatienten führen zu stetigen Weiterentwicklungen in der Linsenchirurgie. Der Wunsch nach einem perfekten Sehvermögen und nach Brillenfreiheit ist ein häufiges Anliegen insbesondere jüngerer Patienten. Die Einführung von Multifokallinsen hat die Therapie der Katarakt revolutioniert. Mit innen kann der Patient sowohl in der Nähe und Ferne als auch im Intermediärbereich scharf sehen. Für die präoperative Berechnung der Linsenstärke sind verschiedene Formeln verfügbar. Bis jetzt wurde keine Studie publiziert, die die Präzision dieser Formeln für die Kalkulation der Stärke von Trioder Quadrifokallinsen untersucht hat. Das Ziel dieser Studie war die Evaluation neun moderner Formeln für die Berechnung der Linsenstärke der quadrifokalen AcrySof IQ PanOptix TFNT00.

\section{Methoden:}

Die vorliegende Studie ist eine retrospektive konsekutive Fallserie, die an der Augenklinik der Johann Wolfgang Goethe-Universität Frankfurt am Main durchgeführt wurde. Es wurden alle Patienten eingeschlossen, die sich einer Femtosekundenlaser-assistierten Operation mit Implantation der quadrifokalen Intraokularlinse unterzogen. Mit dem IOLMaster 500 wurden die präoperativen Biometrie- und Keratometriemessungen durchgeführt. Das Scheimpflug-System Pentacam wurde zur Bestimmung der zentralen Hornhautdicke genutzt. Drei Monate nach der Operation fand eine subjektive Refraktionsbestimmung statt.

Die folgenden Formeln wurden untersucht: Holladay 1, Sanders-Retzlaff-Kraff/ theoretical (SRK/T), Hoffer Q, T2, Holladay 2, Haigis, Barrett Universal II, Olsen und Hill-radiale Basisfunktion (RBF). Aus den präoperativ gemessenen Parametern und der implantierten Linsenstärke wurde für jedes Auge die postoperative Refraktion berechnet. Der Vorhersagefehler (PE) ist definiert als die Differenz zwischen dem tatsächlich erreichten postoperativen sphärischen Äquivalent und dem vorhergesagten postoperativen sphärischen Äquivalent. Zur Reduktion systematischer Fehler und somit des mittleren PE wurden die Linsenkonstanten 
optimiert. Primäre Endpunkte waren die Unterschiede der mittleren absoluten Vorhersagefehler (MAE) zwischen den Formeln. Die Standardabweichung sowie mediane und maximale absolute PE wurden ebenso untersucht wie die Prozentzahlen der Augen, deren PE innerhalb von $\pm 0,25$ Dioptrien (dpt), $\pm 0,5 \mathrm{dpt}$, $\pm 1,0$ dpt und $\pm 2,0$ dpt lagen.

\section{Ergebnisse:}

75 Augen von 38 Patienten wurden in die vorliegende Studie eingeschlossen. Es gab signifikante Unterschiede zwischen den Formeln bezüglich des MAE ( $p$-Wert < 0,001). Die Rangfolge der Formeln entsprechend ihrer MAE lautet wie folgt: Barrett Universal II (0,294 dpt), Hill-RBF (0,332 dpt), Olsen (0,339 dpt), T2 (0,351 dpt), Holladay 1 (0,381 dpt), Haigis (0,382 dpt), SRK/T (0,393 dpt), Holladay $2(0,399 \mathrm{dpt})$ und Hoffer Q (0,410 dpt). Den niedrigsten maximalen absoluten PE erreichte die Barrett Universal II. Bei der Verwendung der Haigis, Barrett Universal II, Olsen und Hill-RBF hatten $80 \%$ der Augen einen PE innerhalb von $\pm 0,5 \mathrm{dpt}$. In der Subgruppe der kurzen Augen wurde der niedrigste PE mit der Hill-RBF erreicht, in der Subgruppe der langen Augen mit der Barrett Universal II und mit der Olsen. Die Unterschiede zwischen den Formeln in den Subgruppenanalysen waren jedoch nicht signifikant.

\section{Schlussfolgerung:}

Die exaktesten Vorhersagen der postoperativen Refraktion konnten mit der Barrett Universal II erzielt werden. Diese Formel sollte daher künftig für die Berechnung der Linsenstärke der quadrifokalen PanOptix genutzt werden. Die T2, Olsen und Hill-RBF führten ebenfalls zu niedrigen PE. Weitere Studien sollten durchgeführt werden, um insbesondere die neuen Formeln im Zusammenhang mit verschiedenen Multifokallinsen zu untersuchen. Um die Vorhersagegenauigkeit der Formeln in Augen mit extremen Achsenlängen zu bewerten, sind Studien mit größeren Fallzahlen nötig. 


\section{Summary}

Background and purpose of the study:

The increase of patients' expectations leads to further developments in cataract surgery. Perfect visual acuity and spectacle independence is often desired especially by relatively young patients. The introduction of multifocal lenses has revolutionized the performance of cataract surgery. They enable an excellent refractive outcome at the near and far as well as at the intermediate position. Different formulas are available for the preoperative lens power calculation. To date, no study has been published evaluating the performance of these formulas for trifocal or quadrifocal intraocular lenses. The aim of this study was to evaluate nine modern formulas used to calculate the lens power of the quadrifocal AcrySof IQ PanOptix TFNT00.

\section{Methods:}

This is a retrospective consecutive case series study performed at the Department of Ophthalmology, Johann Wolfgang Goethe-University Frankfurt. All patients that have undergone femtosecond laser-assisted surgery with implantation of the quadrifocal intraocular lens were enrolled. Preoperative biometry and keratometry measurements were obtained from an IOLMaster 500. The Scheimpflug camera Pentacam was used to measure the central corneal thickness. Subjective refraction measurements were performed three months after the operation.

The following formulas were assessed: Holladay 1, Sanders-Retzlaff-Kraff/ theoretical (SRK/T), Hoffer Q, T2, Holladay 2, Haigis, Barrett Universal II, Olsen, and Hill-radial basis function (RBF). Preoperative measurements and the power of the implanted lens were used to calculate the postoperative refraction for each eye. The refractive prediction error $(\mathrm{PE})$ is defined as the difference between the measured postoperative spherical equivalent and the predicted postoperative spherical equivalent. The lens constants were optimized in order to reduce systematic errors and thus the mean PE. The primary outcomes were differences in mean absolute prediction errors (MAE) between the formulas. Standard 
deviations, median and maximum absolute PE were assessed as well as percentages of eyes within PE of \pm 0.25 dioptres (dpt), $\pm 0.5 \mathrm{dpt}, \pm 1.0 \mathrm{dpt}$, and $\pm 2.0 \mathrm{dpt}$.

\section{Results:}

75 eyes of 38 patients were enrolled in this study. The differences in MAE between the formulas were statistically significant ( $p$-value $<0.001$ ). The ranking of the formulas according to the MAE is: Barrett Universal II (0.294 dpt), Hill-RBF (0.332 dpt), Olsen (0.339 dpt), T2 (0.351 dpt), Holladay 1 (0.381 dpt), Haigis (0.382 dpt), SRK/T (0.393 dpt), Holladay 2 (0.399 dpt), and Hoffer Q (0.410 dpt). The lowest maximum absolute PE was reached using the Barrett Universal II. A PE within $\pm 0.5 \mathrm{dpt}$ was obtained for $80 \%$ of the eyes using the Haigis, Barrett Universal II, Olsen, and Hill-RBF. In the subgroup analysis of short eyes, the lowest MAE was achieved with the Hill-RBF, in the subgroup of long eyes with the Barrett Universal II and Olsen. The differences between the formulas in the subgroup analyses were not statistically significant.

\section{Conclusion:}

The most accurate predictions of postoperative refraction were achieved using the Barrett Universal II. Hence, this formula should be used for the lens power calculation of the quadrifocal PanOptix. The T2, Olsen, and Hill-RBF also resulted in low PE. Especially for the evaluation of modern formulas, further trials in which various multifocal lenses are implanted should be conducted. Studies with larger study populations are necessary in order to appraise the prediction accuracy of the formulas in eyes with extreme axial lengths. 


\section{Literaturverzeichnis}

1. Olson RJ. Cataract surgery from 1918 to the present and future-just imagine! Am J Ophthalmol. 2018;185:10-13.

2. Bourne RRA, Stevens GA, White RA, et al. Causes of vision loss worldwide, 1990-2010: a systematic analysis. Lancet Glob Health. 2013;1(6):e339-349. doi:10.1016/S2214-109X(13)70113-X.

3. Jetter D. Geschichte der Medizin: Einführung in die Entwicklung der Heilkunde aller Länder und Zeiten. Stuttgart: Thieme; 1992.

4. Tarkkanen A. Blindness of Johann Sebastian Bach. Acta Ophthalmol. 2013;91(2):191-192.

5. Jaffe NS. History of cataract surgery. Ophthalmology. 1996;103(8) (suppl):5-16.

6. Ridley $\mathrm{H}$. Intra-ocular acrylic lenses: a recent development in the surgery of cataract. Br J Ophthalmol. 1952;36(3):113-122.

7. Kelman CD. Phaco-emulsification and aspiration: a new technique of cataract removal: a preliminary report. Am J Ophthalmol. 1967;64(1):23-35.

8. Institut für angewandte Qualitätsförderung und Forschung im Gesundheitswesen $\mathrm{GmbH}$. Kataraktoperation: Abschlussbericht. http://sqg.de/upload/CONTENT/NeueVerfahren/Kataraktoperationen/Abschlussbericht_Kataraktoperation.pdf. Accessed July 10, 2019.

9. Grehn F. Augenheilkunde. Berlin: Springer; 2008.

10. Thompson J, Lakhani N. Cataracts. Prim Care. 2015;42(3):409-423.

11. Huppelsberg J, Walter K, eds. Kurzlehrbuch Physiologie. 4th ed. Stuttgart: Thieme; 2013.

12. Baumeister M, Kohnen T. Akkommodation und Presbyopie: Teil 1: Physiologie der Akkommodation und Entwicklung der Presbyopie. Ophthalmologe. 2008;105(6):597-608.

13. Kohnen T, ed. Refraktive Chirurgie. Berlin: Springer; 2011.

14. Atchison DA, Thibos LN. Optical models of the human eye. Clin Exp Optom. 2016;99(2):99-106.

15. Glasser A, Campbell MC. Presbyopia and the optical changes in the human crystalline lens with age. Vision Res. 1998;38(2):209-229. 
16. Song S, Landsbury A, Dahm R, Liu Y, Zhang Q, Quinlan RA. Functions of the intermediate filament cytoskeleton in the eye lens. $J$ Clin Invest. 2009;119(7):1837-1848.

17. Lüllmann-Rauch R, ed. Taschenlehrbuch Histologie. 4th ed. Stuttgart: Thieme; 2012.

18. Olson RJ, Braga-Mele R, Chen SH, et al. Cataract in the adult eye preferred practice pattern. Ophthalmology. 2017;124(2):1-119.

19. Kohnen T, Derhartunian V. Apodisierte Diffraktionsoptik: neues Konzept in der Multifokallinsentechnologie. Ophthalmologe. 2007;104(10):899-907.

20. Truscott RJW, Friedrich MG. The etiology of human age-related cataract: proteins don't last forever. Biochim Biophys Acta. 2016;1860(1 Pt B):192198.

21. Chang JR, Koo E, Agrón E, et al. Risk factors associated with incident cataracts and cataract surgery in the age-related eye disease study (AREDS): AREDS report number 32. Ophthalmology. 2011;118(11):21132119.

22. Richter GM, Torres M, Choudhury F, Azen SP, Varma R, Los Angeles Latino Eye Study Group. Risk factors for cortical, nuclear, posterior subcapsular, and mixed lens opacities: the Los Angeles Latino eye study. Ophthalmology. 2012;119(3):547-554.

23. Shiels A, Hejtmancik JF. Mutations and mechanisms in congenital and age-related cataracts. Exp Eye Res. 2017;156:95-102.

24. McCarty CA, Nanjan MB, Taylor HR. Attributable risk estimates for cataract to prioritize medical and public health action. Invest Ophthalmol Vis Sci. 2000;41(12):3720-3725.

25. Sedaghat F, Ghanavati M, Nezhad Hajian P, Hajishirazi S, Ehteshami M, Rashidkhani B. Nutrient patterns and risk of cataract: a case-control study. Int J Ophthalmol. 2017;10(4):586-592.

26. Zhao L, Chen X-J, Zhu J, et al. Lanosterol reverses protein aggregation in cataracts. Nature. 2015;523(7562):607-611.

27. Berufsverband der Augenärzte Deutschlands e.V., Deutsche Ophthalmologische Gesellschaft e.V. Leitlinie Nr. 19: Katarakt (Grauer Star) im Erwachsenenalter. http://augeninfo.de/leit/leit19.pdf. Accessed August 14, 2017. 
28. Müller M, Kohnen T. Inzisionen für die biaxiale und koaxiale mikroinzisionale Kataraktchirurgie. Ophthalmologe. 2010;107(2):108-115.

29. Menapace R. Entwicklungen der modernen Kataraktchirurgie - eine Übersicht und Bewertung. Ther Umsch. 2016;73(2):53-59.

30. Kolb CM, Shajari M, Mathys $L$, et al. Comparison of femtosecond laserassisted cataract surgery and conventional cataract surgery: meta-analysis and systematic review. J Cataract Refract Surg. Published April 30, 2020. Accessed April 30, 2020. doi:10.1097/j.jcrs.0000000000000228.

31. de Silva SR, Riaz Y, Evans JR. Phacoemulsification with posterior chamber intraocular lens versus extracapsular cataract extraction (ECCE) with posterior chamber intraocular lens for age-related cataract. Cochrane Database Syst Rev. 2014;(1):CD008812. doi:10.1002/14651858.CD008812.pub2.

32. Abell RG, Kerr NM, Vote BJ. Toward zero effective phacoemulsification time using femtosecond laser pretreatment. Ophthalmology. 2013;120(5):942-948.

33. Mayer WJ, Klaproth OK, Hengerer FH, Kohnen T. Impact of crystalline lens opacification on effective phacoemulsification time in femtosecond laserassisted cataract surgery. Am J Ophthalmol. 2014;157(2):426-432.e1. doi:10.1016/j.ajo.2013.09.017.

34. Langenbucher $A$, Viestenz $A$, Szentmáry $N$, Behrens-Baumann $W$, Viestenz A. Toric intraocular lenses-theory, matrix calculations, and clinical practice. J Refract Surg. 2009;25(7):611-622.

35. Kwong YYY, Yuen HKL, Lam RF, Lee VYW, Rao SK, Lam DSC. Comparison of outcomes of primary scleral-fixated versus primary anterior chamber intraocular lens implantation in complicated cataract surgeries. Ophthalmology. 2007;114(1):80-85.

36. Condon GP, Masket S, Kranemann C, Crandall AS, Ahmed IIK. Smallincision iris fixation of foldable intraocular lenses in the absence of capsule support. Ophthalmology. 2007;114(7):1311-1318.

37. Suto C. Sliding scale of IOL power for sulcus fixation using computer simulation. J Cataract Refract Surg. 2004;30(11):2452-2454. 
38. Chang DF, Masket S, Miller KM, et al. Complications of sulcus placement of single-piece acrylic intraocular lenses: recommendations for backup IOL implantation following posterior capsule rupture. J Cataract Refract Surg. 2009;35(8):1445-1458.

39. Wallin T, Parker J, Jin Y, Kefalopoulos G, Olson RJ. Cohort study of 27 cases of endophthalmitis at a single institution. J Cataract Refract Surg. 2005;31(4):735-741.

40. Herrinton LJ, Shorstein NH, Paschal JF, et al. Comparative effectiveness of antibiotic prophylaxis in cataract surgery. Ophthalmology. 2016;123(2):287294.

41. Speaker MG, Milch FA, Shah MK, Eisner W, Kreiswirth BN. Role of external bacterial flora in the pathogenesis of acute postoperative endophthalmitis. Ophthalmology. 1991;98(5):639-650.

42. Carrim ZI, Mackie G, Gallacher G, Wykes WN. The efficacy of $5 \%$ povidone-iodine for 3 minutes prior to cataract surgery. Eur J Ophthalmol. 2009;19(4):560-564.

43. Alio JL, Plaza-Puche AB, Férnandez-Buenaga R, Pikkel J, Maldonado M. Multifocal intraocular lenses: an overview. Surv Ophthalmol. 2017;62(5):611-634 .

44. Findl O, Buehl W, Bauer P, Sycha T. Interventions for preventing posterior capsule opacification. Cochrane Database Syst Rev. 2010;(2):CD003738. doi:10.1002/14651858.CD003738.pub3.

45. Berufsverband der Augenärzte Deutschlands e.V., Deutsche Ophthalmologische Gesellschaft e.V. Leitlinie Nr. 19b: Nd:YAG-Laser Kapsulotomie des Nachstars. http://augeninfo.de/leit/leit19b.pdf. Accessed August 14, 2017.

46. Buehl W, Menapace R, Findl O, Neumayer T, Bolz M, Prinz A. Long-term effect of optic edge design in a silicone intraocular lens on posterior capsule opacification. Am J Ophthalmol. 2007;143(6):913-919.

47. Menapace R. Posterior capsulorhexis combined with optic buttonholing: an alternative to standard in-the-bag implantation of sharp-edged intraocular lenses? A critical analysis of 1000 consecutive cases. Graefes Arch Clin Exp Ophthalmol. 2008;246(6):787-801. 
48. Hoffer KJ. The Hoffer $Q$ formula: a comparison of theoretic and regression formulas. J Cataract Refract Surg. 1993;19(6):700-712.

49. Kohnen T, Kook D, Auffarth GU, Derhartunian V. Einsatzmöglichkeiten intraokularer Multifokallinsen und Kriterien der Patientenselektion. Ophthalmologe. 2008;105(6):527-532.

50. Aristodemou $P$, Cartwright N, Sparrow JM, Johnston RL. Improving refractive outcomes in cataract surgery: a global perspective. World $\mathrm{J}$ Ophthalmol. 2014;4(4):140-146.

51. Nowroozzadeh $\mathrm{MH}$, Mirhosseini A, Meshkibaf MH, Roshannejad J. Effect of Ramadan fasting in tropical summer months on ocular refractive and biometric characteristics. Clin Exp Optom. 2012;95(2):173-176.

52. The Royal College of Ophthalmologists. Cataract Surgery Guidelines. http://rcophth.ac.uk/wp-content/uploads/2014/12/2010-SCI-069-CataractSurgery-Guidelines-2010-SEPTEMBER-2010-1.pdf. Accessed July 11, 2019.

53. Mayer E, Cadman D, Ewings $P$, et al. A 10 year retrospective survey of cataract surgery and endophthalmitis in a single eye unit: injectable lenses lower the incidence of endophthalmitis. Br J Ophthalmol. 2003;87(7):867869.

54. Liekfeld A, Befurt D. Sonderlinsen in der refraktiven Chirurgie. Klin Monatsbl Augenheilkd. 2016;233(8):914-922.

55. Olsen T. Calculation of intraocular lens power: a review. Acta Ophthalmol Scand. 2007;85(5):472-485.

56. Morales EL, Rocha KM, Chalita MR, Nosé W, Avila MP. Comparison of optical aberrations and contrast sensitivity between aspheric and spherical intraocular lenses. J Refract Surg. 2011;27(10):723-728.

57. de Silva SR, Evans JR, Kirthi V, Ziaei M, Leyland M. Multifocal versus monofocal intraocular lenses after cataract extraction. Cochrane Database Syst Rev. 2016;12:CD003169. doi:10.1002/14651858.CD003169.pub4.

58. Su P-Y, Hu F-R. Intraindividual comparison of functional vision and higher order aberrations after implantation of aspheric and spherical intraocular lenses. J Refract Surg. 2009;25(3):265-272.

59. Pieh S. Intraokulare Multifokallinsen. Spektrum Augenheilkd. 2016;30(6):213-216. 
60. Packer M, Fine IH, Hoffman RS. Aspheric intraocular lens selection based on corneal wavefront. J Refract Surg. 2009;25(1):12-20.

61. Alcon. PanOptix Enlightening Conversation.

http://theophthalmologist.com/fileadmin/top/issues/0816/Alcon.pdf Accessed May 31, 2017.

62. Alcon Laboratories Inc. AcrySof IQ PanOptix [package insert]

63. Sudhir RR, Dey A, Bhattacharrya S, Bahulayan A. AcrySof IQ PanOptix intraocular lens versus extended depth of focus intraocular lens and trifocal intraocular lens: a clinical overview. Asia Pac J Ophthalmol (Phila). 2019;8(4):335-349.

64. Auffarth GU, Rabsilber TM, Kohnen T, Holzer MP. Design und optische Prinzipien von Multifokallinsen. Ophthalmologe. 2008;105(6):522-526.

65. Xu X, Zhu M-M, Zou H-D. Refractive versus diffractive multifocal intraocular lenses in cataract surgery: a meta-analysis of randomized controlled trials. $J$ Refract Surg. 2014;30(9):634-644.

66. Hirnschall N, Gangwani V, Crnej A, Koshy J, Maurino V, Findl O. Correction of moderate corneal astigmatism during cataract surgery: toric intraocular lens versus peripheral corneal relaxing incisions. J Cataract Refract Surg. 2014;40(3):354-361.

67. Ferreira TB, Marques EF, Rodrigues A, Montés-Micó R. Visual and optical outcomes of a diffractive multifocal toric intraocular lens. J Cataract Refract Surg. 2013;39(7):1029-1035.

68. Zhou H, Zhu C, Xu W, Zhou F. The efficacy of accommodative versus monofocal intraocular lenses for cataract patients: a systematic review and meta-analysis. Medicine (Baltimore). 2018;97(40):e12693.

doi:10.1097/MD.0000000000012693.

69. Nuzzi R, Tridico F. Comparison of visual outcomes, spectacles dependence and patient satisfaction of multifocal and accommodative intraocular lenses: innovative perspectives for maximal refractive-oriented cataract surgery. BMC Ophthalmol. 2017;17:12. doi:10.1186/s12886-0170411-9.

70. Alió JL, Alió Del Barrio JL, Vega-Estrada A. Accommodative intraocular lenses: where are we and where we are going. Eye Vis (Lond). 2017;4:16. doi:10.1186/s40662-017-0077-7. 
71. Braga-Mele R, Chang D, Dewey S, et al. Multifocal intraocular lenses: relative indications and contraindications for implantation. J Cataract Refract Surg. 2014;40(2):313-322.

72. Kook D. Biometrie vor Linsenchirurgie. Presented at the: Forum für angewandte Bildgebung in der Ophthalmologie 2018; April 22, 2018; München.

73. Zvorničanin J, Zvorničanin E. Premium intraocular lenses: the past, present and future. J Curr Ophthalmol. 2018;30(4):287-296.

74. IOL Innovations ApS. PhacoOptics Manual. http://phacooptics.net/product/phacooptics-manual/. Accessed July 20, 2019.

75. Grotz B. Optische Geräte. http://grund-wissen.de/physik/optik/optischegeraete.html. Accessed August 17, 2017.

76. Haigis $W$. Challenges and approaches in modern biometry and IOL calculation. Saudi J Ophthalmol. 2012;26(1):7-12.

77. Norrby S. Sources of error in intraocular lens power calculation. J Cataract Refract Surg. 2008;34(3):368-376.

78. Haag-Streit AG. Lenstar LS 900. http://haag-streit.com/de/haag-streitdeutschland/produkte/hs-diagnostics/biometrie/lenstar-ls-900/. Accessed August 20, 2017.

79. Carl Zeiss Meditec AG. IOLMaster with Advanced Technology Software Version 5.4 User Manual. http://doctorhill.com/physicians/docs/iolmaster_5-4.pdf. Accessed June 13, 2017.

80. Buckhurst PJ, Wolffsohn JS, Shah S, Naroo SA, Davies LN, Berrow EJ. A new optical low coherence reflectometry device for ocular biometry in cataract patients. Br J Ophthalmol. 2009;93(7):949-953.

81. Reitblat O, Levy A, Kleinmann G, Assia El. Accuracy of intraocular lens power calculation using three optical biometry measurement devices: the OA-2000, Lenstar-LS900 and IOLMaster-500. Eye (Lond). 2018;32(7):1244-1252.

82. Reitblat O, Assia El, Kleinmann G, Levy A, Barrett GD, Abulafia A. Accuracy of predicted refraction with multifocal intraocular lenses using two biometry measurement devices and multiple intraocular lens power calculation formulas. Clin Exp Ophthalmol. 2015;43(4):328-334. 
83. OCULUS. Basissoftware Pentacam. http://pentacam.com/de/augenarztoperativ-ohne-pentacamr/modelle/pentacamr/basissoftware.html. Accessed July 10, 2019.

84. Holladay JT. Standardizing constants for ultrasonic biometry, keratometry, and intraocular lens power calculations. J Cataract Refract Surg. 1997;23(9):1356-1370.

85. Haigis $\mathrm{W}$. The keratometer index problem. http://ocusoft.de/ulib/czm/texte/kprobl/kprobl.htm. Accessed May 30, 2017.

86. Hoffer KJ. Definition of ACD. Ophthalmology. 2011;118(7):1484.

87. Retzlaff JA, Sanders DR, Kraff MC. Development of the SRK/T intraocular lens implant power calculation formula. J Cataract Refract Surg. 1990;16(3):333-340.

88. Holladay JT, Prager TC, Chandler TY, Musgrove KH, Lewis JW, Ruiz RS. A three-part system for refining intraocular lens power calculations. $J$ Cataract Refract Surg. 1988;14(1):17-24.

89. ZEISS Medical Technology. IOL Power Calculation Formulas Explained. http://cataract-community.zeiss.com/iol-calculation-formulas-explained. Accessed July 11, 2019.

90. Aristodemou P, Knox Cartwright NE, Sparrow JM, Johnston RL. Intraocular lens formula constant optimization and partial coherence interferometry biometry: Refractive outcomes in 8108 eyes after cataract surgery. $J$ Cataract Refract Surg. 2011;37(1):50-62.

91. Hill WE, Abulafia A, Wang L, Koch DD. Pursuing perfection in IOL calculations: II. measurement foibles: measurement errors, validation criteria, IOL constants, and lane length. J Cataract Refract Surg. 2017;43(7):869-870.

92. Colenbrander MC. Calculation of the power of an iris clip lens for distant vision. Br J Ophthalmol. 1973;57(10):735-740.

93. Binkhorst RD. The optical design of intraocular lens implants. Ophthalmic Surg. 1975;6(3):17-31.

94. Fyodorov SN, Galin MA, Linksz A. Calculation of the optical power of intraocular lenses. Invest Ophthalmol. 1975;14(8):625-628.

95. Hoffer KJ. Intraocular lens calculation: the problem of the short eye. Ophthalmic Surg. 1981;12(4):269-272. 
96. Sanders D, Retzlaff $J, K$ raff M, et al. Comparison of the accuracy of the Binkhorst, Colenbrander, and SRK implant power prediction formulas. $J$ Am Intraocul Implant Soc. 1981;7(4):337-340.

97. Sanders DR, Retzlaff J, Kraff MC. Comparison of the SRK II formula and other second generation formulas. J Cataract Refract Surg. 1988;14(2):136-141.

98. Sheard RM, Smith GT, Cooke DL. Improving the prediction accuracy of the SRK/T formula: the T2 formula. J Cataract Refract Surg. 2010;36(11):18291834.

99. Koch DD, Hill W, Abulafia A, Wang L. Pursuing perfection in intraocular lens calculations: I. logical approach for classifying IOL calculation formulas. J Cataract Refract Surg. 2017;43(6):717-718.

100. Shammas HJ. Intraocular Lens Power Calculations. Thorofare, NJ: SLACK Incorporated; 2004.

101. Haigis W. IOL-Kalkulation bei hohen Ametropien. Ophthalmologe. 2008;105(11):999-1004.

102. Reitblat O, Levy A, Kleinmann G, Lerman TT, Assia El. Intraocular lens power calculation for eyes with high and low average keratometry readings: comparison between various formulas. J Cataract Refract Surg. 2017;43(9):1149-1156.

103. Haigis W. Intraocular lens calculation in extreme myopia. J Cataract Refract Surg. 2009;35(5):906-911.

104. Barrett GD. Intraocular lens calculation formulas for new intraocular lens implants. J Cataract Refract Surg. 1987;13(4):389-396.

105. Barrett GD. An improved universal theoretical formula for intraocular lens power prediction. J Cataract Refract Surg. 1993;19(6):713-720.

106. Olsen T. Prediction of the effective postoperative (intraocular lens) anterior chamber depth. J Cataract Refract Surg. 2006;32(3):419-424.

107. Olsen T, Corydon L, Gimbel H. Intraocular lens power calculation with an improved anterior chamber depth prediction algorithm. J Cataract Refract Surg. 1995;21(3):313-319.

108. Olsen T, Olesen H, Thim K, Corydon L. Prediction of pseudophakic anterior chamber depth with the newer IOL calculation formulas. J Cataract Refract Surg. 1992;18(3):280-285. 
109. Olsen T, Funding M. Ray-tracing analysis of intraocular lens power in situ. J Cataract Refract Surg. 2012;38(4):641-647.

110. Olsen T, Hoffmann P. C constant: new concept for ray tracing-assisted intraocular lens power calculation. J Cataract Refract Surg. 2014;40(5):764-773.

111. IOL Innovations ApS. PhacoOptics Program Overview. http://phacooptics.net/program-overview/. Accessed July 11, 2019.

112. Cooke DL, Cooke TL. Comparison of 9 intraocular lens power calculation formulas. J Cataract Refract Surg. 2016;42(8):1157-1164.

113. Haag-Streit AG. EyeSuite IOL. http://haag-streit.com/de/haag-streitdeutschland/produkte/hs-diagnostics/biometrie/eyesuite-iol/. Accessed July $11,2019$.

114. Kane J. Kane Formula. http://iolformula.com/about/. Accessed July 11, 2019.

115. Yeo TK. EVO Formula. http://evoiolcalculator.com/. Accessed July 11, 2019.

116. Ferreira TB, Ribeiro P, Ribeiro FJ, O'Neill JG. Comparison of astigmatic prediction errors associated with new calculation methods for toric intraocular lenses. J Cataract Refract Surg. 2017;43(3):340-347.

117. Savini G, Hoffer KJ. Intraocular lens power calculation in eyes with previous corneal refractive surgery. Eye Vis (Lond). 2018;5:18. doi:10.1186/s40662-018-0110-5

118. American Society of Cataract and Refractive Surgery. IOL power calculator in post-myopic LASIK/PRK eyes. http://iolcalc.ascrs.org/. Accessed July $11,2019$.

119. American Society of Cataract and Refractive Surgery. Barrett Toric Calculator. http://ascrs.org/barrett-toric-calculator. Accessed July 11, 2019. 120. Kane JX, Van Heerden A, Atik A, Petsoglou C. Intraocular lens power formula accuracy: comparison of 7 formulas. J Cataract Refract Surg. 2016;42(10):1490-1500.

121. Kane JX, Van Heerden A, Atik A, Petsoglou C. Accuracy of 3 new methods for intraocular lens power selection. J Cataract Refract Surg. 2017;43(3):333-339. 
122. Alcon Pharma GmbH. Laser-Refraktive Kataraktchirurgie. http://alconpharma.de/produkte/ophthalmochirurgie/copy_of_intrepid. Accessed July 31, 2019.

123. Eyefox UG. AcrySof IQ PanOptix (Toric) TFNT00.

http://eyefox.com/iol/346/acrysof-iq-panoptix-toric-tfnt00.html. Accessed August 5, 2019.

124. Lee S, Choi M, Xu Z, Zhao Z, Alexander E, Liu Y. Optical bench performance of a novel trifocal intraocular lens compared with a multifocal intraocular lens. Clin Ophthalmol. 2016;10:1031-1038.

125. Kohnen T. First implantation of a diffractive quadrafocal (trifocal) intraocular lens. J Cataract Refract Surg. 2015;41(10):2330-2332.

126. Retzlaff JA, Sanders DR, Kraff MC. Erratum. J Cataract Refract Surg. 1990;16:528.

127. Haigis W. Occurrence of erroneous anterior chamber depth in the SRK/T formula. J Cataract Refract Surg. 1993;19(3):442-446.

128. Hoffer KJ. Erratum. J Cataract Refract Surg. 1994;20:677.

129.Zuberbuhler B, Morrell AJ. Errata in printed Hoffer Q formula. J Cataract Refract Surg. 2007;33(1):2.

130. Sheard RM. T2 Formula Calculator 1.3. http://richardsheard.net/T2Formula.aspx. Accessed January 6, 2017.

131. Holladay IOL Consultant Software \& Surgical Outcomes Assessment [computer program]. Version 2018.0120. Bellaire, TX: Holladay Consulting; 2015.

132. Holladay JT. HICSOAP Professional and Deluxe User's Manual. http://hicsoap.com/downloads/pro/5\%20HicPro\%20User's\%20Manual\%20 17dec11.pdf. Accessed May 9, 2017.

133. Haigis W, Lege B, Miller N, Schneider B. Comparison of immersion ultrasound biometry and partial coherence interferometry for intraocular lens calculation according to Haigis. Graefes Arch Clin Exp Ophthalmol. 2000;238(9):765-773.

134. Asia-Pacific Association of Cataract and Refractive Surgeons. Barrett Universal II Formula. http://apacrs.org/barrett_universal2/. Accessed May 10, 2017. 
135. PhacoOptics IOL Power Calculation Software [computer program]. Version 1.10.100.2032. Aarhus: IOL Innovations ApS; 2009.

136. Hill WE. Hill-RBF method. http://rbfcalculator.com/online/index.html. Accessed June 7, 2017.

137. Hill WE, Haag-Streit AG. Hill-RBF Calculator Instructions for Use. http://rbfcalculator.com/docs/Hill-RBF-Calculator-Instructions.pdf. Accessed June 11, 2017.

138. Haigis W. Optimized IOL constants for the ZEISS IOLMaster. http://ocusoft.de/ulib/c1.htm. Accessed May 30, 2017.

139. Wang L, Koch DD, Hill W, Abulafia A. Pursuing perfection in intraocular lens calculations: III. criteria for analyzing outcomes. J Cataract Refract Surg. 2017;43(8):999-1002.

140. Melles RB, Holladay JT, Chang WJ. Accuracy of intraocular lens calculation formulas. Ophthalmology. 2018;125(2):169-178.

141. Liedtke S. Brille ade dank neuester Kunstlinsen. Frankfurter Neue Presse. 2015;238:14.

142. Hoffer KJ, Aramberri J, Haigis W, et al. Protocols for studies of intraocular lens formula accuracy. Am J Ophthalmol. 2015;160(3):403-405.e1. doi:10.1016/j.ajo.2015.05.029.

143. Aristodemou P, Knox Cartwright NE, Sparrow JM, Johnston RL. Statistical analysis for studies of intraocular lens formula accuracy. Am J Ophthalmol. 2015;160(5):1085-1086.

144. Reinstein DZ, Archer TJ, Srinivasan S, et al. Standard for reporting refractive outcomes of intraocular lens-based refractive surgery. J Cataract Refract Surg. 2017;43(4):435-439.

145. Olsen T. On the calculation of power from curvature of the cornea. $\mathrm{Br} \mathrm{J}$ Ophthalmol. 1986;70(2):152-154.

146. Lam S, Gupta BK, Hahn JM, Manastersky NA. Refractive outcomes after cataract surgery: Scheimpflug keratometry versus standard automated keratometry in virgin corneas. J Cataract Refract Surg. 2011;37(11):19841987.

147. Girbardt C, Oertel N, Adamek-Dyk J, Wiedemann P, Nestler A. Refraktive Veränderungen bei Triple-Descemet-Membran-Endothel-Keratoplastik. Ophthalmologe. 2016;113(3):217-222. 
148. Eom Y, Kang S-Y, Song JS, Kim YY, Kim HM. Comparison of Hoffer $Q$ and Haigis formulae for intraocular lens power calculation according to the anterior chamber depth in short eyes. Am J Ophthalmol. 2014;157(4):818824.e2. doi:10.1016/j.ajo.2013.12.017.

149. Ning X, Yang Y, Yan H, Zhang J. Anterior chamber depth - a predictor of refractive outcomes after age-related cataract surgery. BMC Ophthalmol. 2019;19(1):134. doi:10.1186/s12886-019-1144-8.

150. Goto S, Maeda N, Koh S, et al. Prediction of postoperative intraocular lens position with angle-to-angle depth using anterior segment optical coherence tomography. Ophthalmology. 2016;123(12):2474-2480.

151. Shammas HJ, Shammas MC. Improving the preoperative prediction of the anterior pseudophakic distance for intraocular lens power calculation. $J$ Cataract Refract Surg. 2015;41(11):2379-2386.

152. Gale RP, Saldana M, Johnston RL, Zuberbuhler B, McKibbin M. Benchmark standards for refractive outcomes after NHS cataract surgery. Eye (Lond). 2009;23(1):149-152.

153. Iribarren R, Morgan IG, Nangia V, Jonas JB. Crystalline lens power and refractive error. Invest Ophthalmol Vis Sci. 2012;53(2):543-550.

154. Lam S. Comparison of age-derived lens thickness to optically measured lens thickness in IOL power calculation: a clinical study. J Refract Surg. 2012;28(2):154-155.

155. Srivannaboon S, Chirapapaisan C, Chirapapaisan N, Lertsuwanroj B, Chongchareon M. Accuracy of Holladay 2 formula using IOLMaster parameters in the absence of lens thickness value. Graefes Arch Clin Exp Ophthalmol. 2013;251(11):2563-2567.

156. Olsen T. Use of fellow eye data in the calculation of intraocular lens power for the second eye. Ophthalmology. 2011;118(9):1710-1715.

157. Turnbull AMJ, Barrett GD. Using the first-eye prediction error in cataract surgery to refine the refractive outcome of the second eye. J Cataract Refract Surg. 2019;45(9):1239-1245.

158. Muthappan V, Paskowitz D, Kazimierczak A, Jun AS, Ladas J, Kuo IC. Measurement and use of postoperative anterior chamber depth of fellow eye in refractive outcomes. J Cataract Refract Surg. 2015;41(4):778-784. 
159. Martinez-Enriquez E, Pérez-Merino P, Durán-Poveda S, Jiménez-Alfaro I, Marcos S. Estimation of intraocular lens position from full crystalline lens geometry: towards a new generation of intraocular lens power calculation formulas. Sci Rep. 2018;8:9829. doi:10.1038/s41598-018-28272-6.

160. National Institute for Health and Care Excellence. Cataracts in adults: management. http://ncbi.nlm.nih.gov/books/NBK464182/pdf/Bookshelf_NBK464182.pdf. Accessed August 2, 2018.

161. Huang J, McAlinden C, Huang Y, et al. Meta-analysis of optical lowcoherence reflectometry versus partial coherence interferometry biometry. Sci Rep. 2017;7:43414. doi:10.1038/srep43414.

162. Melles RB, Kane JX, Olsen T, Chang WJ. Update on intraocular lens calculation formulas. Ophthalmology. 2019;126(9):1334-1335.

163. Roberts TV, Hodge C, Sutton G, Lawless M, contributors to the Vision Eye Institute IOL outcomes registry. Comparison of Hill-radial basis function, Barrett Universal and current third generation formulas for the calculation of intraocular lens power during cataract surgery. Clin Exp Ophthalmol. 2018;46(3):240-246 .

164. Wallace HB, Misra SL, Li SS, McKelvie J. Predicting pseudophakic refractive error: interplay of biometry prediction error, anterior chamber depth, and changes in corneal curvature. J Cataract Refract Surg. 2018;44(9):1123-1129.

165. Wan KH, Lam TC, Yu MC, Chan TC. Accuracy and precision of intraocular lens calculations using the new Hill-RBF version 2.0 in eyes with high axial myopia. Am J Ophthalmol. 2019;205:66-73.

166. Ramji H, Moore J, Moore CBT, Shah S. Can the accuracy of multifocal intraocular lens power calculation be improved to make patients spectacle free? Cont Lens Anterior Eye. 2016;39(2):160-166.

167. Kim BH, Hyon JY, Kim MK. Effects of bifocal versus trifocal diffractive intraocular lens implantation on visual quality after cataract surgery. Korean J Ophthalmol. 2019;33(4):333-342. 
168. Gökce SE, Montes De Oca I, Cooke DL, Wang L, Koch DD, Al-Mohtaseb Z. Accuracy of 8 intraocular lens calculation formulas in relation to anterior chamber depth in patients with normal axial lengths. $J$ Cataract Refract Surg. 2018;44(3):362-368.

169. Wang $Q$, Jiang W, Lin T, Wu X, Lin H, Chen W. Meta-analysis of accuracy of intraocular lens power calculation formulas in short eyes. Clin Exp Ophthalmol. 2018;46(4):356-363.

170. Wang Q, Jiang W, Lin T, et al. Accuracy of intraocular lens power calculation formulas in long eyes: a systematic review and meta-analysis. Clin Exp Ophthalmol. 2018;46(7):738-749.

171. Hill DC, Sudhakar S, Hill CS, et al. Intraoperative aberrometry versus preoperative biometry for intraocular lens power selection in axial myopia. $J$ Cataract Refract Surg. 2017;43(4):505-510.

172. Wang L, Shirayama M, Ma XJ, Kohnen T, Koch DD. Optimizing intraocular lens power calculations in eyes with axial lengths above $25.0 \mathrm{~mm}$. $J$ Cataract Refract Surg. 2011;37(11):2018-2027.

173. Wang L, Holladay JT, Koch DD. Wang-Koch axial length adjustment for the Holladay 2 formula in long eyes. J Cataract Refract Surg. 2018;44(10):1291-1292.

174. European Society of Cataract and Refractive Surgery. ESCRS 2017 Clinical Trends Survey Results. http://eurotimes.org/escrs-2017-clinicaltrends-survey-results/. Accessed July 10, 2019.

175. Kohnen T, Herzog M, Hemkeppler E, et al. Visual performance of a quadrifocal (trifocal) intraocular lens following removal of the crystalline lens. Am J Ophthalmol. 2017;184:52-62.

176. Monaco G, Gari M, Di Censo F, Poscia A, Ruggi G, Scialdone A. Visual performance after bilateral implantation of 2 new presbyopia-correcting intraocular lenses: trifocal versus extended range of vision. J Cataract Refract Surg. 2017;43(6):737-747.

177. Akman A, Asena L, Ozturk C, Gür Güngör S. Evaluation of quality of life after implantation of a new trifocal intraocular lens. J Cataract Refract Surg. 2019;45(2):130-134. 
178. Martínez de Carneros-Llorente A, Martínez de Carneros A, Martínez de Carneros-Llorente $\mathrm{P}$, Jiménez-Alfaro I. Comparison of visual quality and subjective outcomes among 3 trifocal intraocular lenses and 1 bifocal intraocular lens. J Cataract Refract Surg. 2019;45(5):587-594.

179. Mencucci R, Favuzza E, Caporossi O, Savastano A, Rizzo S. Comparative analysis of visual outcomes, reading skills, contrast sensitivity, and patient satisfaction with two models of trifocal diffractive intraocular lenses and an extended range of vision intraocular lens. Graefes Arch Clin Exp Ophthalmol. 2018;256(10):1913-1922.

180. Lawless M, Hodge C, Reich J, et al. Visual and refractive outcomes following implantation of a new trifocal intraocular lens. Eye Vis (Lond). 2017;4:10. doi:10.1186/s40662-017-0076-8. 


\section{Anhang}

\subsection{Details der publizierten Formeln}

Im Folgenden sind die fünf komplett publizierten Formeln aufgeführt. Die Parameter werden jeweils ohne Einheiten eingesetzt. Trotzdem sind sie der Vollständigkeit halber in den Beschriftungen mit angegeben.

\subsubsection{Holladay 1}

Quelle: Holladay et al ${ }^{88}$

\subsubsection{Schätzung der postoperativen Vorderkammertiefe}

$$
\begin{gathered}
r=\frac{337,5}{K} \\
\text { Falls } r<7, \text { dann } r_{\text {kor }}=7 \\
\text { Falls } r \geq 7, \text { dann } r_{\text {kor }}=r
\end{gathered}
$$

Formel 6: $K=$ Hornhautbrechkraft [Dioptrien], $r=$ Hornhautradius [mm], $r_{k o r}=$ korrigierter Hornhautradius [mm]

$$
\begin{gathered}
V K D=\frac{12,5 A L}{23,45} \\
\text { Falls } V K D>13,5 \text {, dann } V K D=13,5
\end{gathered}
$$

Formel 7: AL = Achsenlänge [mm], VKD = Vorderkammerdurchmesser $[\mathrm{mm}]$

$$
\begin{gathered}
S F=(0,5663 A)-65,60 \\
A C D_{\text {post }}=r_{\text {kor }}-\sqrt{r_{\text {kor }}{ }^{2}-\frac{V K D^{2}}{4}}+S F+0,56
\end{gathered}
$$

Formel 8: $A=A$-Konstante, $A C D_{\text {post }}=$ geschätzte postoperative Vorderkammertiefe $[\mathrm{mm}], r_{\mathrm{kor}}=$ korrigierter Hornhautradius [mm], $S F=$ Surgeon Factor, $V K D=$ Vorderkammerdurchmesser [mm] 


\subsubsection{Berechnung der Linsenstärke und Vorhersage der Refraktion}

$$
V=12 ; n_{a}=1,336 ; n_{c}=\frac{4}{3} ; R T=0,2
$$

Formel 9: $n_{a}=$ Kammerwasserbrechungsindex, $n_{c}=$ Hornhautbrechungsindex, $R T=$ Retinadicke [mm], $V=$ Scheitelabstand einer Brille [mm]

$$
A L_{\text {mod }}=A L+R T
$$

Formel 10: $A L=$ Achsenlänge $[\mathrm{mm}], A L_{\text {mod }}=$ modifizierte Achsenlänge $[\mathrm{mm}], R T=$ Retinadicke [mm]

Formel 10 ist nur für den Fall relevant, dass die AL mit Ultraschall gemessen wurde. Andernfalls, d. h. bei der Messung mit optischer Biometrie, fällt sie weg. Die gemessene $A L$ wird dann direkt als $A L_{\bmod }$ eingesetzt.

$$
L P=\frac{1000 n_{a}\left(n_{a} r-\left[n_{c}-1\right] A L_{m o d}-0,001 \operatorname{Ref}\left[V\left(n_{a} r-\left[n_{c}-1\right] A L_{m o d}\right)+A L_{\text {mod }} r\right]\right)}{\left(A L_{\text {mod }}-A C D_{\text {post }}\right)\left(n_{a} r-\left[n_{c}-1\right] A C D_{\text {post }}-0,001 \operatorname{Ref}\left[V\left(n_{a} r-\left[n_{c}-1\right] A C D_{\text {post }}\right)+A C D_{\text {post }} r\right]\right)}
$$

Formel 11: $A C D_{\text {post }}=$ geschätzte postoperative Vorderkammertiefe $[\mathrm{mm}], A L_{\text {mod }}=$ modifizierte Achsenlänge [mm], LP = Linsenstärke [Dioptrien], $n_{a}=$ Kammerwasserbrechungsindex, $n_{c}=$ Hornhautbrechungsindex, $r=$ Hornhautradius [mm], Ref = Zielrefraktion [Dioptrien], $V=$ Scheitelabstand einer Brille [mm]

Ref

$=\frac{1000 n_{a}\left(n_{a} r-\left[n_{c}-1\right] A L_{\text {mod }}\right)-L P\left(A L_{\text {mod }}-A C D_{\text {post }}\right)\left(n_{a} r-\left[n_{c}-1\right] A C D_{\text {post }}\right)}{n_{a}\left(V\left[n_{a} r-\left(n_{c}-1\right) A L_{\text {mod }}\right]+A L_{\text {mod }} r\right)-0,001 L P\left(A L_{\text {mod }}-A C D_{\text {post }}\right)\left(V\left[n_{a} r-\left(n_{c}-1\right) A C D_{\text {post }}\right]+A C D_{\text {post }} r\right)}$

Formel 12: $A C D_{\text {post }}=$ geschätzte postoperative Vorderkammertiefe $[\mathrm{mm}], A L_{\text {mod }}=$ modifizierte Achsenlänge [mm], LP = Linsenstärke [Dioptrien], $n_{a}=$ Kammerwasserbrechungsindex, $n_{c}=$ Hornhautbrechungsindex, $r=$ Hornhautradius $[\mathrm{mm}]$, Ref $=$ vorhergesagte postoperative Refraktion [Dioptrien], $V=$ Scheitelabstand einer Brille [mm] 


\subsubsection{Sanders-Retzlaff-Kraff/theoretical}

Quellen: Retzlaff et al, ${ }^{87,126}$ Haigis $^{127}$

\subsubsection{Schätzung der postoperativen Vorderkammertiefe}

$$
\text { Falls } A L \leq 24,2 \text {, dann } A L_{\text {kor }}=A L
$$

$$
\text { Falls } A L>24,2 \text {, dann } A L_{\text {kor }}=-3,446+1,716 A L-0,0237 A L^{2}
$$

Formel 13: $A L=$ Achsenlänge $[\mathrm{mm}], A L_{\text {kor }}=$ korrigierte Achsenlänge $[\mathrm{mm}]$

$$
\begin{gathered}
r=\frac{337,5}{K} \\
W T W=-5,40948+0,58412 A L_{k o r}+0,098 K \\
x=r^{2}-\frac{W T W^{2}}{4} \\
\text { Falls } x<0, \text { dann } x=0 \\
H=r-\sqrt{x}
\end{gathered}
$$

Formel 14: $A L_{\text {kor }}=$ korrigierte Achsenlänge $[\mathrm{mm}], H=$ Hornhauthöhe $[\mathrm{mm}], K=$ Hornhautbrechkraft [Dioptrien], $r=$ Hornhautradius [mm], WTW = Hornhautdurchmesser [mm]

$$
\begin{gathered}
A C D_{\text {Konst }}=0,62467 A-68,747 \\
\text { Offset }=A C D_{\text {Konst }}-3,336 \\
A C D_{\text {post }}=H+\text { Offset }
\end{gathered}
$$

Formel 15: $A=A$-Konstante, $A C D_{\text {Konst }}=$ Konstante der Vorderkammertiefe, $A C D_{\text {post }}=$ geschätzte postoperative Vorderkammertiefe [mm], $\mathrm{H}=$ Hornhauthöhe $[\mathrm{mm}]$ 


\subsubsection{Berechnung der Linsenstärke und Vorhersage der Refraktion}

$$
V=12 ; n_{a}=1,336 ; n_{c}=1,333
$$

Formel 16: $n_{a}=$ Kammerwasserbrechungsindex, $n_{c}=$ Hornhautbrechungsindex, $V=$ Scheitelabstand einer Brille [mm]

$$
\begin{gathered}
R T=0,65696-0,02029 \mathrm{AL} \\
A L_{o p t}=A L+R T
\end{gathered}
$$

Formel 17: $A L=$ Achsenlänge $[\mathrm{mm}], A L_{\text {opt }}=$ optische Achsenlänge $[\mathrm{mm}], R T=$ Retinadicke $[\mathrm{mm}]$

Formel 17 ist nur für den Fall relevant, dass die AL mit Ultraschall gemessen wurde. Andernfalls, d. h. bei der Messung mit optischer Biometrie, fällt sie weg. Die gemessene $A L$ wird dann direkt als $A L_{\text {opt }}$ eingesetzt.

$$
L P=\frac{1000 n_{a}\left(n_{a} r-\left[n_{c}-1\right] A L_{o p t}-0,001 \operatorname{Ref}\left[V\left(n_{a} r-\left[n_{c}-1\right] A L_{o p t}\right)+A L_{o p t} r\right]\right)}{\left(A L_{o p t}-A C D_{\text {post }}\right)\left(n_{a} r-\left[n_{c}-1\right] A C D_{\text {post }}-0,001 \operatorname{Ref}\left[V\left(n_{a} r-\left[n_{c}-1\right] A C D_{\text {post }}\right)+A C D_{\text {post }} r\right]\right)}
$$

Formel 18: $A C D_{\text {post }}=$ geschätzte postoperative Vorderkammertiefe $[\mathrm{mm}], A L_{\text {opt }}=$ optische Achsenlänge [mm], LP = Linsenstärke [Dioptrien], $n_{a}=$ Kammerwasserbrechungsindex, $n_{c}=$ Hornhautbrechungsindex, $r=$ Hornhautradius [mm], Ref $=$ Zielrefraktion [Dioptrien], $V=$ Scheitelabstand einer Brille [mm]

Ref

$=\frac{1000 n_{a}\left(n_{a} r-\left[n_{c}-1\right] A L_{\text {opt }}\right)-L P\left(A L_{\text {opt }}-A C D_{\text {post }}\right)\left(n_{a} r-\left[n_{c}-1\right] A C D_{\text {post }}\right)}{n_{a}\left(V\left[n_{a} r-\left(n_{c}-1\right) A L_{\text {opt }}\right]+A L_{\text {opt }} r\right)-0,001 L P\left(A L_{\text {opt }}-A C D_{\text {post }}\right)\left(V\left[n_{a} r-\left(n_{c}-1\right) A C D_{\text {post }}\right]+A C D_{\text {post }} r\right)}$

Formel 19: $A C D_{\text {post }}=$ geschätzte postoperative Vorderkammertiefe $[\mathrm{mm}], A L_{\text {opt }}=$ optische Achsenlänge [mm], LP = Linsenstärke [Dioptrien], $n_{a}=$ Kammerwasserbrechungsindex, $n_{c}=$ Hornhautbrechungsindex, $r=$ Hornhautradius $[\mathrm{mm}]$, Ref $=$ vorhergesagte postoperative Refraktion [Dioptrien], $V=$ Scheitelabstand einer Brille [mm] 


\subsubsection{Hoffer $Q$}

Quellen: Hoffer, ${ }^{48,128}$ Zuberbuhler und Morrell ${ }^{129}$

\subsubsection{Schätzung der postoperativen Vorderkammertiefe}

$$
\begin{gathered}
\text { Falls } A L<18,5 \text {, dann } A L=18,5 \\
\text { Falls } A L>31 \text {, dann } A L=31 \\
\text { Falls } A L \leq 23 \text {, dann } x=1 \text { und } y=28 \\
\text { Falls } A L>23 \text {, dann } x=-1 \text { und } y=23,5 \\
A C D_{\text {post }}=p A C D+0,3(A L-23,5)+(\tan K)^{2} \\
+0,1 \times(23,5-A L)^{2}\left(\tan \left[0,1(y-A L)^{2}\right]\right)-0,99166
\end{gathered}
$$

Formel 20: $A C D_{\text {post }}=$ geschätzte postoperative Vorderkammertiefe $[\mathrm{mm}], A L=$ Achsenlänge [mm], $p A C D=$ personalisierte Vorderkammertiefe, $K=$ Hornhautbrechkraft [Dioptrien]

\subsubsection{Berechnung der Linsenstärke und Vorhersage der Refraktion}

Die Limitationen bezüglich der AL aus Formel 20 sind für die Berechnung der Linsenstärke nicht mehr gültig.

$$
\begin{gathered}
V=12, n_{c}=1,336 \\
L P=\frac{1000 n_{c}}{A L-A C D_{\text {post }}-0,05}-\left(\frac{n_{c}}{\frac{n_{c}}{K+\frac{R e f}{1-\frac{V}{1000} \text { Ref }}}-\frac{A C D_{\text {post }}+0,05}{1000}}\right)
\end{gathered}
$$

Formel 21: $A C D_{\text {post }}=$ geschätzte postoperative Vorderkammertiefe $[\mathrm{mm}], A L=$ Achsenlänge [mm], LP = Linsenstärke [Dioptrien], $K=$ Hornhautbrechkraft [Dioptrien], $n_{c}=$ Hornhautbrechungsindex, Ref $=$ Zielrefraktion [Dioptrien], $V=$ Scheitelabstand einer Brille [mm] 


$$
\operatorname{Ref}=\frac{\frac{n_{c}}{\frac{n_{c}}{A L-A C D_{\text {post }}-0,05}-L P}+\frac{A C D_{\text {post }}+0,05}{1000}-K}{1+\frac{V}{1000}\left(\frac{n_{c}}{\left.\frac{1000 n_{c}}{\frac{n_{c}}{A L-A C D_{\text {post }}-0,05}-L P}+\frac{A C n_{\text {post }}+0,05}{1000}-K\right)}\right)}
$$

Formel 22: $A C D_{\text {post }}=$ geschätzte postoperative Vorderkammertiefe $[\mathrm{mm}], A L=$ Achsenlänge [mm], LP = Linsenstärke [Dioptrien], $K=$ Hornhautbrechkraft [Dioptrien], $n_{c}=$ Hornhautbrechungsindex, Ref = vorhergesagte postoperative Refraktion [Dioptrien], $V=$ Scheitelabstand einer Brille [mm]

\subsection{4. $\underline{\text { T2 }}$}

Quelle: Sheard et al ${ }^{98}$

Die T2 ist eine Überarbeitung der SRK/T. Aus diesem Grund werden nur die Änderungen aufgeführt.

\subsubsection{1. Änderung der Berechnung der korrigierten Achsenlänge}

Ersetzen von Formel 13 durch Folgendes:

$$
\text { Falls } A L \leq 24,2 \text {, dann } A L_{\text {kor }}=A L
$$

$$
\begin{aligned}
& \text { Falls } 36,2>A L>24,2 \text {, dann } A L_{\text {kor }}=-3,446+1,716 A L-0,0237 A L^{2} \\
& \text { Falls } A L \geq 36,2 \text {, dann } A L_{\text {kor }}=-3,446+1,716 * 36,2-0,0237 * 36,2^{2}
\end{aligned}
$$

Formel 23: $A L=$ Achsenlänge $[\mathrm{mm}], A L_{\text {kor }}=$ korrigierte Achsenlänge $[\mathrm{mm}]$

Das Ersetzen dieses Berechnungsschrittes ist letztendlich nicht von Bedeutung, da die endgültige T2 ihn nicht mehr miteinbezieht. 


\subsubsection{2. Änderung der Berechnung der Hornhauthöhe}

Ersetzen von Formel 13 und 14 durch Folgendes:

$$
H=0,32630 A L+0,13533 K-10,326
$$

Formel 24: $A L=$ Achsenlänge [mm], $H=$ Hornhauthöhe [mm], $K=$ Hornhautbrechkraft [Dioptrien]

\subsubsection{Haigis}

Quelle: Haigis et al ${ }^{133}$

\subsubsection{Schätzung der postoperativen Vorderkammertiefe}

$$
\begin{aligned}
n_{c} & =1,3315 \\
K & =\frac{n_{c}-1}{r}
\end{aligned}
$$

Formel 25: $n_{c}=$ Hornhautbrechungsindex, $r=$ Hornhautradius $[\mathrm{mm}], K=$ Hornhautbrechkraft [Dioptrien]

$$
\begin{gathered}
A C D_{\text {Mittel }}=3,37 ; A L_{\text {Mittel }}=23,39 \\
A C D_{\text {Konst }}=0,62467 A-68,747 \\
a 0=A C D_{\text {Konst }}-a 1 * A C D_{\text {Mittel }}-a 2 * A L_{\text {Mittel }} \\
A C D_{\text {post }}=a 0+a 1 * A C D_{\text {prä }}+a 2 * A L_{\text {prä }}
\end{gathered}
$$

Formel 26: $A=A$-Konstante, $A C D_{\text {Mittel }}=$ Mittelwert der Vorderkammertiefe $[\mathrm{mm}], A C D_{\text {Konst }}=$ Konstante der Vorderkammertiefe, $A C D_{\text {post }}=$ geschätzte postoperative Vorderkammertiefe [mm], $A C D_{\text {prä }}=$ präoperative Vorderkammertiefe $[\mathrm{mm}], A L_{\text {prä }}=$ präoperative Achsenlänge $[\mathrm{mm}], A L_{\text {Mittel }}=$ Mittelwert der Achsenlänge [mm] 


\subsubsection{Berechnung der Linsenstärke und Vorhersage der Refraktion}

$$
V=12 ; n_{a}=1,336
$$

Formel 27: $n_{a}=$ Kammerwasserbrechungsindex, $V=$ Scheitelabstand einer Brille [mm $]$

$$
L P=\frac{n_{a}}{A L-A C D_{\text {post }}}-\frac{n_{a}}{K+\frac{n_{a}}{1-\operatorname{Ref} * V}}-A C D_{\text {post }}
$$

Formel 28: $A C D_{\text {post }}=$ geschätzte postoperative Vorderkammertiefe $[\mathrm{mm}], A L=$ Achsenlänge [mm], LP = Linsenstärke [Dioptrien], $n_{a}=$ Kammerwasserbrechungsindex, Ref $=$ Zielrefraktion [Dioptrien], $V=$ Scheitelabstand einer Brille [mm]

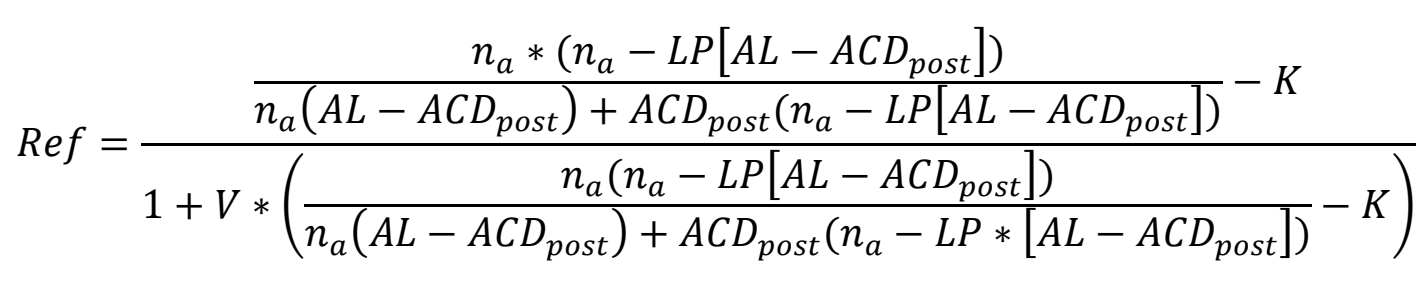

Formel 29: $A C D_{\text {post }}=$ geschätzte postoperative Vorderkammertiefe $[\mathrm{mm}], A L=$ Achsenlänge [mm], LP = Linsenstärke [Dioptrien], $K=$ Hornhautbrechkraft [Dioptrien], $n_{a}=$ Kammerwasserbrechungsindex, Ref $=$ vorhergesagte postoperative Refraktion [Dioptrien], $V=$ Scheitelabstand einer Brille [mm] 


\subsection{Ergebnisse der Cochran Q-Tests der Gesamtanalyse}

Tabelle 13: Vergleich der Anteile der Augen innerhalb eines Vorhersagefehlers von $\pm 0,25 d p t$ (+ = signifikanter Unterschied, - = kein signifikanter Unterschied, Bar = Barrett Universal II, Hill = Hill-RBF, Hoffer $=$ Hoffer $Q$, Holl $1=$ Holladay 1 , Holl $2=$ Holladay 2)

\begin{tabular}{|l|c|c|c|c|c|c|c|c|c|}
\hline & Holl 1 & SRK/T & Hoffer & T2 & Holl 2 & Haigis & Bar & Olsen & Hill \\
\hline Holl 1 & & - & - & - & - & - & + & + & + \\
\hline SRK/T & - & & - & - & - & - & + & + & - \\
\hline Hoffer & - & - & & + & + & + & + & + & + \\
\hline T2 & - & - & + & & - & - & - & - & - \\
\hline Holl 2 & - & - & + & - & & - & - & - & - \\
\hline Haigis & - & - & + & - & - & & - & - & - \\
\hline Bar & + & + & + & - & - & - & & - & - \\
\hline Olsen & + & + & + & - & - & - & - & & - \\
\hline Hill & + & - & + & - & - & - & - & - & \\
\hline
\end{tabular}

Tabelle 14: Vergleich der Anteile der Augen innerhalb eines Vorhersagefehlers von $\pm 0,5 d p t$ (+ = signifikanter Unterschied, - = kein signifikanter Unterschied, Bar = Barrett Universal II, Hill = Hill-RBF, Hoffer $=$ Hoffer $Q$, Holl $1=$ Holladay 1 , Holl $2=$ Holladay 2$)$

\begin{tabular}{|l|c|c|c|c|c|c|c|c|c|}
\hline & Holl 1 & SRK/T & Hoffer & T2 & Holl 2 & Haigis & Bar & Olsen & Hill \\
\hline Holl 1 & & - & - & - & - & - & - & - & - \\
\hline SRK/T & - & & - & - & - & + & + & + & + \\
\hline Hoffer & - & - & & - & - & - & - & - & - \\
\hline T2 & - & - & - & & - & - & - & - & - \\
\hline Holl 2 & - & - & - & - & & + & + & + & + \\
\hline Haigis & - & + & - & - & + & & - & - & - \\
\hline Bar & - & + & - & - & + & - & & - & - \\
\hline Olsen & - & + & - & - & + & - & - & & - \\
\hline Hill & - & + & - & - & + & - & - & - & \\
\hline
\end{tabular}




\subsection{Vergleiche von jeweils zwei Formeln innerhalb der Subgruppen}

Tabelle 15: P-Werte der Vergleiche von jeweils zwei Formeln (Subgruppe mit Achsenlänge $<22,5 \mathrm{~mm}$ )

(Bar = Barrett Universal II, Hill $=$ Hill-RBF, Hoffer $=$ Hoffer $Q$, Holl $1=$ Holladay 1, Holl $2=$ Holladay 2)

\begin{tabular}{|l|r|r|r|r|r|r|r|r|r|}
\hline & Holl 1 & SRK/T & Hoffer & T2 & Holl 2 & Haigis & Bar & \multicolumn{1}{l|}{ Olsen } & \multicolumn{1}{l|}{ Hill } \\
\hline Holl 1 & - & 0,953 & 0,021 & 0,953 & 0,515 & 0,214 & 0,374 & 0,515 & 0,314 \\
\hline SRK/T & 0,953 & - & 0,021 & 0,767 & 0,441 & 0,214 & 0,594 & 0,314 & 0,678 \\
\hline Hoffer & 0,021 & 0,021 & - & 0,008 & 0,767 & 0,678 & 0,173 & 0,953 & 0,066 \\
\hline T2 & 0,953 & 0,767 & 0,008 & - & 0,441 & 0,173 & 0,441 & 0,260 & 0,314 \\
\hline Holl 2 & 0,515 & 0,441 & 0,767 & 0,441 & - & 0,214 & 0,859 & 0,859 & 0,110 \\
\hline Haigis & 0,214 & 0,214 & 0,678 & 0,173 & 0,214 & - & 0,314 & 0,038 & 0,110 \\
\hline Bar & 0,374 & 0,594 & 0,173 & 0,441 & 0,859 & 0,314 & - & 0,594 & 0,075 \\
\hline Olsen & 0,515 & 0,314 & 0,953 & 0,260 & 0,859 & 0,038 & 0,594 & - & 0,314 \\
\hline Hill & 0,314 & 0,678 & 0,066 & 0,314 & 0,110 & 0,110 & 0,075 & 0,314 & - \\
\hline
\end{tabular}

Tabelle 16: P-Werte der Vergleiche von jeweils zwei Formeln (Subgruppe mit Achsenlänge $>25,5 \mathrm{~mm}$ )

(Bar $=$ Barrett Universal II, Hill $=$ Hill-RBF, Hoffer $=\operatorname{Hoffer} Q$, Holl $1=$ Holladay 1, Holl $2=$ Holladay 2)

\begin{tabular}{|l|r|r|r|r|r|r|r|r|r|}
\hline & Holl 1 & SRK/T & Hoffer & T2 & Holl 2 & Haigis & Bar & Olsen & \multicolumn{1}{l|}{ Hill } \\
\hline Holl 1 & - & 0,594 & 0,028 & 0,051 & 0,051 & 0,011 & 0,021 & 0,021 & 0,038 \\
\hline SRK/T & 0,594 & - & 0,086 & 0,038 & 0,173 & 0,051 & 0,015 & 0,021 & 0,021 \\
\hline Hoffer & 0,028 & 0,086 & - & 0,594 & 0,173 & 0,767 & 0,594 & 0,374 & 0,953 \\
\hline T2 & 0,051 & 0,038 & 0,594 & - & 0,214 & 0,767 & 0,594 & 0,214 & 0,314 \\
\hline Holl 2 & 0,051 & 0,173 & 0,173 & 0,214 & - & 0,214 & 0,021 & 0,021 & 0,139 \\
\hline Haigis & 0,011 & 0,051 & 0,767 & 0,767 & 0,214 & - & 0,374 & 0,374 & 0,953 \\
\hline Bar & 0,021 & 0,015 & 0,594 & 0,594 & 0,021 & 0,374 & - & 0,314 & 0,063 \\
\hline Olsen & 0,021 & 0,021 & 0,374 & 0,214 & 0,021 & 0,374 & 0,314 & - & 0,050 \\
\hline Hill & 0,038 & 0,021 & 0,953 & 0,314 & 0,139 & 0,953 & 0,063 & 0,050 & - \\
\hline
\end{tabular}




\section{Eigene Veröffentlichungen}

Shajari M, Kolb CM, Petermann K, et al. Comparison of 9 modern intraocular lens power calculation formulas for a quadrifocal intraocular lens. J Cataract Refract Surg. 2018;44(8):942-948.

Kolb CM. Vergleich von konventionellem und beschleunigtem Crosslinking zur Behandlung von Keratokonus - eine Metaanalyse. DOG-Kongressausgabe der Ophthalmologischen Nachrichten. 2018;1:5-6.

Shajari M, Kolb CM, Agha B, et al. Comparison of standard and accelerated corneal cross-linking for the treatment of keratoconus: a meta-analysis. Acta Ophthalmol. 2019;97(1):e22-e35. doi:10.1111/aos.13814.

Shajari M, Kolb CM, Mayer WJ, et al. Characteristics of preoperative and postoperative astigmatism in patients having Descemet membrane endothelial keratoplasty. J Cataract Refract Surg. 2019;45(7):1001-1006.

Kolb CM, Shajari M, Mathys L, et al. Comparison of femtosecond laser-assisted cataract surgery and conventional cataract surgery: meta-analysis and systematic review. J Cataract Refract Surg. Published April 30, 2020. Accessed April 30, 2020. doi:10.1097/j.jcrs.0000000000000228. 


\section{Lebenslauf}

Carolin Marion Kolb

Geboren am 2. August 1995 in Aschaffenburg

\section{Ausbildung}

06/ 2014

Erwerb der allgemeinen Hochschulreife am Friedrich

Dessauer-Gymnasium, Aschaffenburg

Seit 10/ 2014 Studium der Humanmedizin an der Johann Wolfgang

Goethe-Universität, Frankfurt am Main

$09 / 2016$

Erster Abschnitt der ärztlichen Prüfung

2018-2020

Förderung durch das Deutschlandstipendium

$04 / 2020$

Zweiter Abschnitt der ärztlichen Prüfung

\section{Famulaturen}

2016

Klinik für Augenheilkunde, Aschaffenburg

2017

Praxis für Hals-Nasen-Ohrenheilkunde, Aschaffenburg

2017

Praxis für Anästhesie, Aschaffenburg

2018

Praxis für innere Medizin, Aschaffenburg

2018

Klinik für Augenheilkunde, Universität München

2019

Klinik für Neurologie, Universität Frankfurt a. M.

2019

Klinik für Augenheilkunde, Universität Mainz

2019

Klinik für Augenheilkunde, Universität Frankfurt a. M.

\section{Praktisches Jahr}

1. Tertial Chirurgie, St. Katharinen-Krankenhaus, Frankfurt a. M.

2. Tertial Augenheilkunde, Universitätsklinikum, Frankfurt a. M.

3. Tertial Innere Medizin, St. Katharinen-Krankenhaus, Frankfurt a. M. 


\section{Danksagung}

An erster Stelle danke ich meinem Doktorvater Prof. Dr. Thomas Kohnen für die Überlassung des Dissertationsthemas und für seine Förderung bei der Mitarbeit an weiteren Fragestellungen.

Mein besonderer Dank gilt meinem Betreuer Dr. Mehdi Shajari für die hervorragende Unterstützung und ständige Erreichbarkeit über den gesamten Zeitraum der Entstehung dieser Arbeit und für die Publikation der Studie im Journal of Cataract and Refractive Surgery. Ich möchte mich außerdem bedanken, dass er mir die Möglichkeit gab, meine Ergebnisse auf dem Kongress der Deutschen Ophthalmologischen Gesellschaft 2018 in Bonn vorzustellen.

Kerstin Petermann und Lisa Hinzelmann bin ich dankbar für ihre organisatorische Hilfe.

Dem Verein der Alumni und Freunde des Fachbereichs Medizin e.V. sowie der Stiftung Giersch danke ich für ihre Förderung im Rahmen des Deutschlandstipendiums.

Danke an Denise Hundshammer, ohne die ich nie den Mut aufgebracht hätte, englische Publikationen zu schreiben.

Ich danke Beatrice Grebe für ihr offenes Ohr, ihre guten Ratschläge und das Korrekturlesen meiner Dissertation.

Besonders möchte ich mich bei Martin Wetterau bedanken, der verständnisvoll und zu jeder Zeit in allen Höhen und Tiefen für mich da war und mich aufgemuntert hat.

Der größte Dank gilt meinen Eltern und Großeltern für ihren Zuspruch, ihre Liebe und ihr Vertrauen in mich und alle meine Vorhaben. 


\section{Schriftliche Erklärung}

Ich erkläre ehrenwörtlich, dass ich die dem Fachbereich Medizin der Johann Wolfgang Goethe-Universität Frankfurt am Main zur Promotionsprüfung eingereichte Dissertation mit dem Titel

Vergleich von neun modernen Formeln zur Berechnung der Linsenstärke einer quadrifokalen Intraokularlinse

in der Klinik für Augenheilkunde

unter Betreuung und Anleitung von Prof. Dr. Thomas Kohnen mit Unterstützung durch Dr. Mehdi Shajari ohne sonstige Hilfe selbst durchgeführt und bei der Abfassung der Arbeit keine anderen als die in der Dissertation angeführten Hilfsmittel benutzt habe. Darüber hinaus versichere ich, nicht die Hilfe einer kommerziellen Promotionsvermittlung in Anspruch genommen zu haben.

Ich habe bisher an keiner in- oder ausländischen Universität ein Gesuch um Zulassung zur Promotion eingereicht. Die vorliegende Arbeit wurde bisher nicht als Dissertation eingereicht.

Vorliegende Ergebnisse der Arbeit wurden in folgendem Publikationsorgan veröffentlicht:

Shajari M, Kolb CM, Petermann K, Böhm M, Herzog M, de'Lorenzo N, Schönbrunn S, Kohnen T. Comparison of 9 modern intraocular lens power calculation formulas for a quadrifocal intraocular lens. Journal of Cataract and Refractive Surgery. Band 44, Seiten 942-948, 2018. 